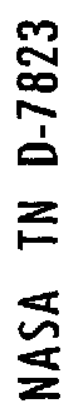

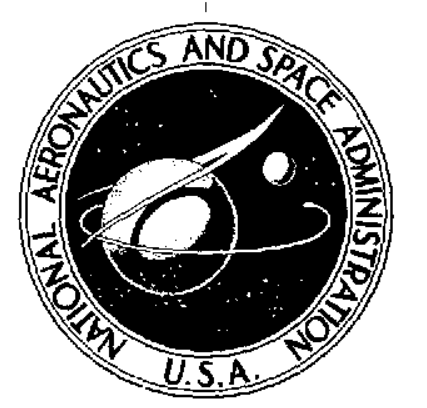

NASA TN D-7823

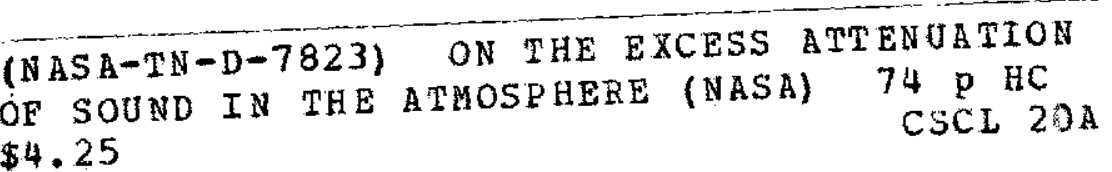

\title{
ON THE EXCESS ATTENUATION OF SOUND IN THE ATMOSPHERF
}

Richard DeLoach

Langley Research Center Hampton, Va. 23665

NATIONAL aERONAUTICS AND SPACE ADMINISTRATION - WASHINGTON, D. C. - MARCH 1975 


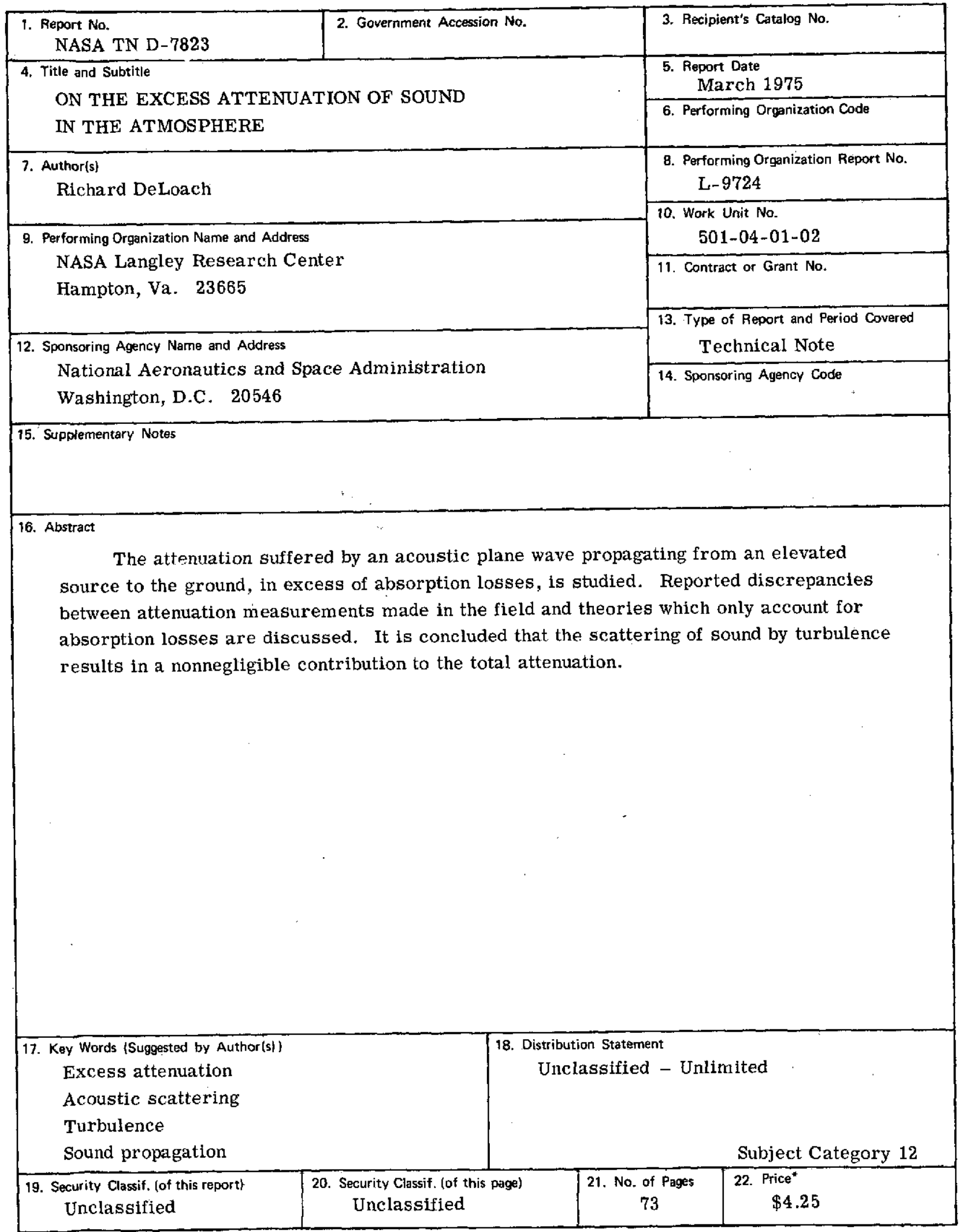

"For sale by the National Technical Information Service, Springfield, Virginia 22151 


\section{CONTENTS}

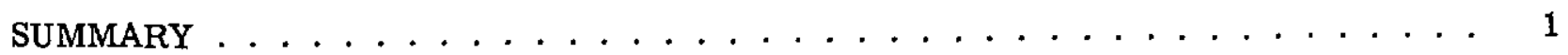

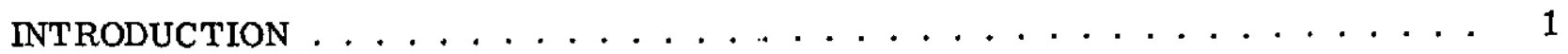

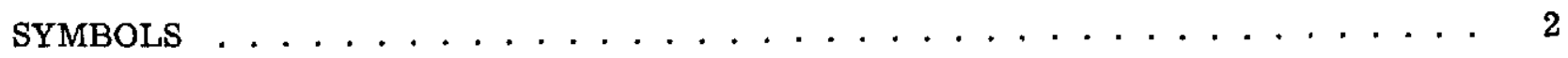

GENERAL DESCRIPTION OF ATTENUATION PROBLEM . . . . . . . . . . . . 6

EMPIRICAL DESCRIPTION OF EXCESS ATTENUATION . . . . . . . . . . . 7

Magnitude ........................... . . . 8

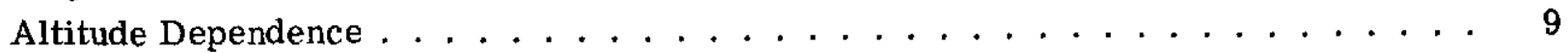

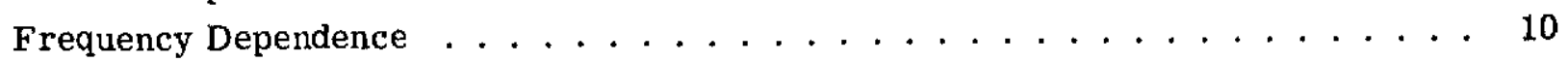

Windspeed Gradients . . . . . . . . . . . . . . . . . . . . 11

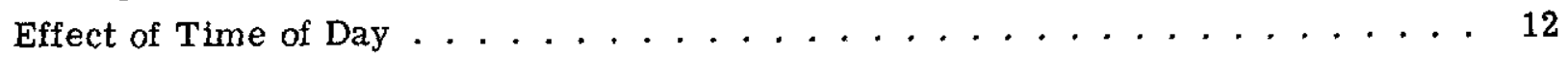

Summary of Previous Experimental Results . . . . . . . . . . . . . . . . . . . 12

POTENTIAL MECHANISMS FOR EXCESS ATTENUATION . . . . . . . . . . . . . . . 12

Refraction ........................ 13

Ground Attenuation . . . . . . . . . . . . . . . . . . . . . 13

Effects of Dust. . . . . . . . . . . . . . . . . . . . . 14

Effects of Fog ........................... . . . 14

Relaxation Processes ........................ 15

Effects of Turbulence . . . . . . . . . . . . . . . . . . . . 15

BRIEF REVIEW OF EARLIER THEORIES, FOUNDATION, AND APPROACH . . . . . 15

PRESENT DEVELOPMENT . . . . . . . . . . . . . . . . . . . . . . 18

Turbulence Model . . . . . . . . . . . . . . . . . . . 18

Theory . . . . . . . . . . . . . . . . . . . . . 20

APPLICATION OF THE THEORY AND A COMPARISON WITH

EXPERIMENTAL RESULTS. . . . . . . . . . . . . . . . . . . . . 29

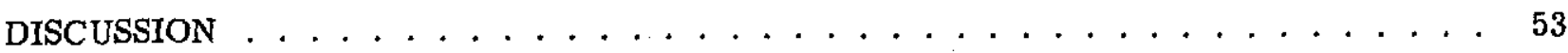

The Angle $\theta_{c} \ldots \ldots \ldots \ldots \ldots \ldots$

Limits of Applicability of Theory . . . . . . . . . . . . . . . . . . 56

Application of the Theory to the Solution of Aircraft Noise

Measurement Problems. . . . . . . . . . . . . . . . 57

CONCLUSIONS . . . . . . . . . . . . . . . . . . . . . . . 58

APPENDIX A - EVALUATION OF THE EXCESS ATTENUATION INTEGRAL . . . . 61

APPENDIX B - A REPRESENTATION OF THE STRUCTURE CONSTANTS

IN TERMS OF FLUCTUATIONS IN WIND AND TEMPERATURE . . . . . . . . . . 64

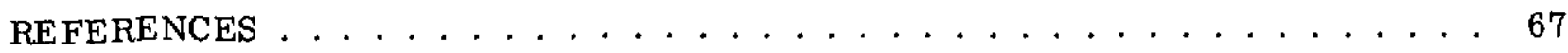




\title{
ON THE EXCESS ATTENUATION OF -SOUND IN THE ATMOSPHERE
}

\author{
By Richard DeLoach \\ Langley Research Center
}

\section{SUMMARY}

The attenuation suffered by a sound wave propagating from an elevated source to the ground, in excess of spherical spreading losses and classical and molecular absorption effects, is studied. Reported discrepancies between attenuation measurements made in the field and the predictions of theories based only on absorption effects are discussed. It is concluded that atmospheric dynamics (turbulence) is a major contributor to the attenuation of sound waves propagating vertically in the atmosphere.

Earlier theories on the attenuation of sound due to scattering which have been addressed separately to either the problem of inertial scattering induced by momentum fluctuations or the problem of scattering induced by temperature fluctuations have led to predictions of a strong (square-law) frequency dependence and relatively large magnitudes which are not supported by the available field data. The present theory considers both inertial and thermal scattering and predicts a square-law frequency dependence only for the special case of a medium for which the scattering of sound can be described as a simple Bragg diffraction process. In a medium for which the Bragg condition represents only a first approximation to the scattering process, a much milder frequency dependence is predicted which approaches a cube root dependence for the case of an outer scale of turbulence that is large compared with half an acoustic wavelength (the general case for audio frequencies in the atmosphere). This prediction is supported by field data, as are the excess attenuation magnitudes predicted by the present theory.

The theory is applied to explain the results of certain field measurements and laboratory experiments which previous theories have not explained. Potential applications of the theory to the solution of aircraft noise measurement problems are discussed.

\section{INTRODUCTION}

The recent introduction of stringent aircraft noise certification requirements by the FAA and a general reemphasis on acoustics research and development priorities at the national level have enhanced the need for greater precision in evaluating the results of aircraft noise measurements. In order to achieve the desired precision, it is necessary to correct raw aircraft noise data accurately to reference meteorological conditions, since the attenuation of sound in the atmosphere can depend quite sensitively on local atmospheric conditions. 
Standard values of atmospheric attenuation for evaluating aircraft noise were published in 1964 by the Society of Automotive Engineers (SAE) as Aerospace Recommended Practice 866 (ARP 866) and have been widely used since that time to correct aircraft noise measurements. (See ref. 1.) These standards are based on the theoretical work of Kneser (ref. 2) as modified by the laboratory experiments of Harris (ref. 3). The results of Kneser and Harris can only be applied rigorously to the description of sound attenuation in still air in a controlled environment; there are additional attenuation mechanisms unaccounted for in their work which come into play when noise measurements are made in the free atmosphere. These mechanisms, which result in what has come to be called the "excess" attenuation, were not well understood when the SAE published reference 1 in 1964. The results of Kneser and Harris were therefore altered, based on aircraft noise data then available, in an attempt to compensate empirically for the excess attenuation.

In recent years there have been occasions when the use of ARP 866 has resulted in data exhibiting certain ambiguities. These developments should not have been wholly unanticipated since ARP 866 has its roots in a theory which does not include all the attenuation mechanisms which are active in the atmosphere. In particular, the problem of acoustic interactions with turbulent density fluctuations in the atmosphere is not treated in the Kneser theory.

In this paper, the theory of sound attenuation in the free atmosphere is reexamined, particular attention being paid to the effects of atmospheric turbulence on the transmission of sound. A formula is derived for the excess attenuation coefficient which depends on the structure constants which characterize atmospheric turbulence and a technique for measuring these structure constants is discussed briefly. The theory is applied to explain certain general features of the excess attenuation which have not been explained by previous theories and potential applications of the theory to aircraft noise measurement problems are discussed.

\section{SYMBOLS}

A

cross-sectional area of a volume element

$\mathrm{A}_{\mathrm{S}} \quad$ accumulated excess attenuation

a,b empirical constants

$\mathrm{C}_{\mathrm{T}} \quad$ temperature structure constant 


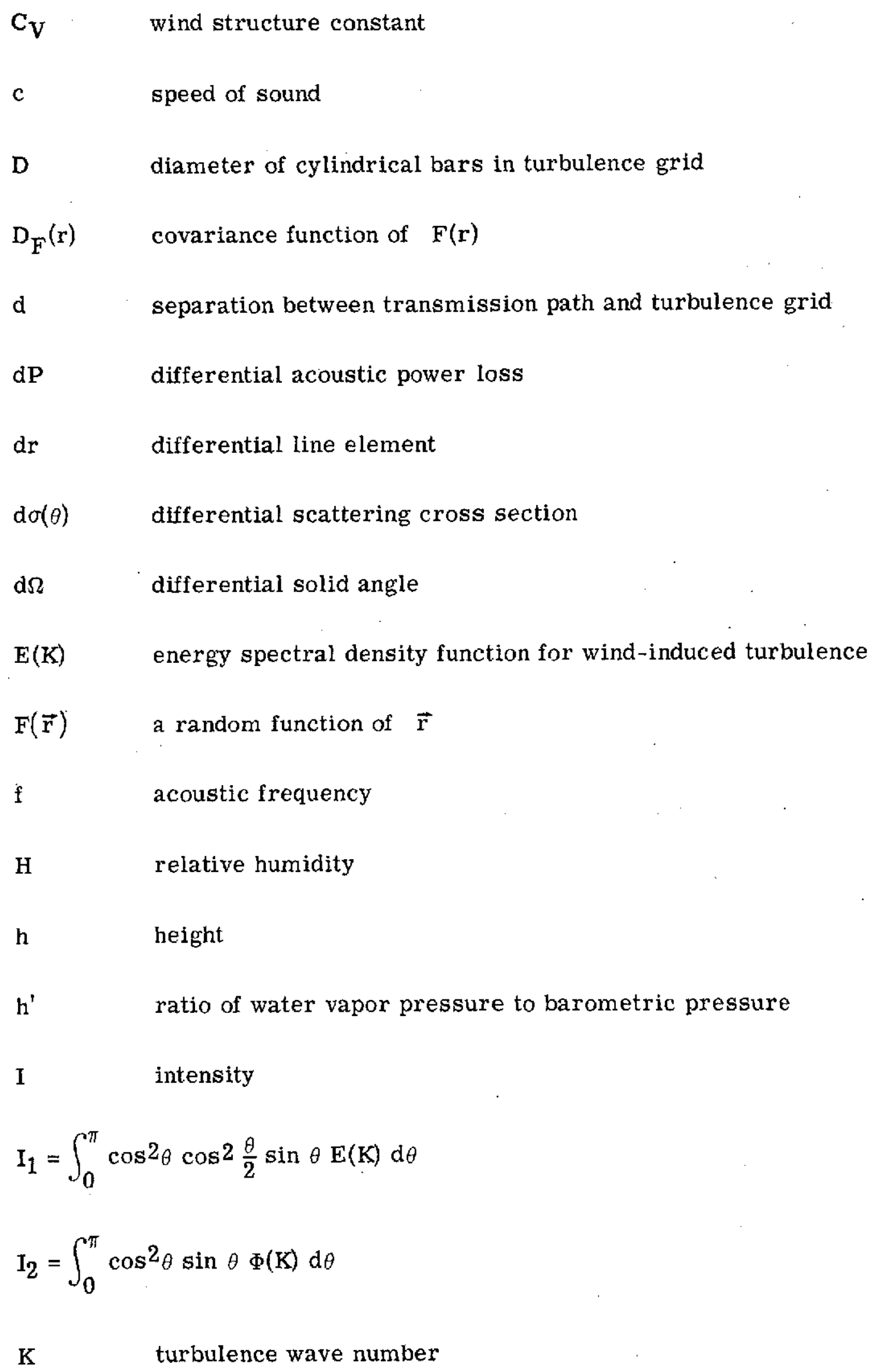




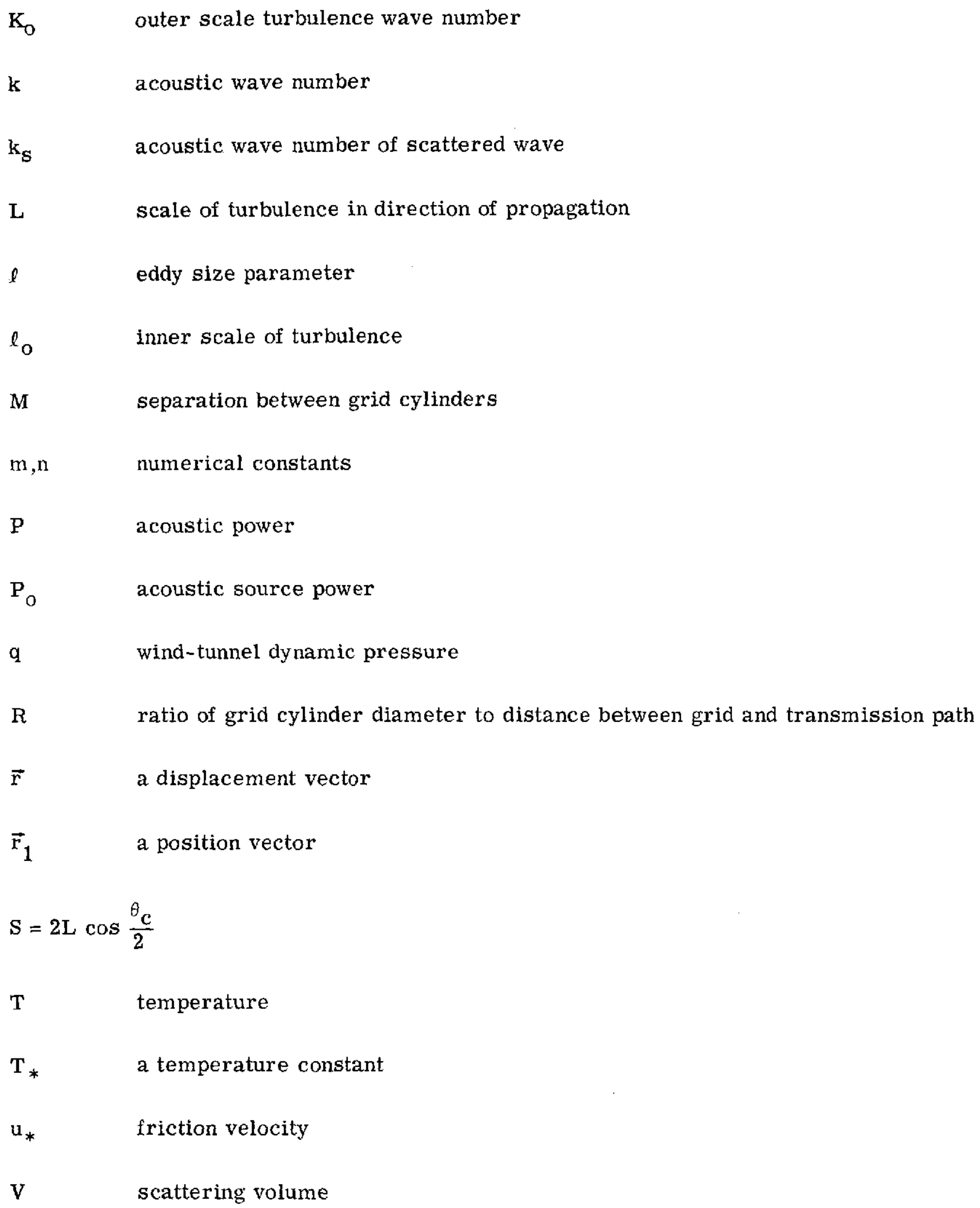


$\mathrm{y}=\sin \frac{\theta}{2}$

$\mathrm{y}_{\mathrm{o}}=\frac{\mathrm{K}_{\mathrm{o}}}{2 \mathrm{k}}$

$\alpha \quad$ attenuation coefficient

$\alpha_{\mathrm{c}} \quad$ classical absorption coefficient

$\alpha_{\mathrm{m}} \quad$ molecular absorption coefficient

$\alpha_{\mathrm{s}} \quad$ coefficient of attenuation due to scattering

$\Gamma \quad$ gamma function

$\gamma=\xi \mathrm{K}_{\mathrm{O}}^{-2 / 3}$

$\Delta \mathrm{F} \quad$ fluctuation of function $\mathrm{F}$ about its mean value

$\overline{\Delta \mathrm{T}^{2}} \quad$ mean-square temperature fluctuation

$\overline{\Delta \mathrm{v}^{2}} \quad$ mean-square velocity fluctuation

$\theta \quad$ Bragg scattering angle

$\theta_{\mathrm{c}} \quad$ difference between true scattering angle and Bragg scattering angle

$\theta_{0} \quad$ smallest angle through which sound of a given frequency will scatter when encountering an eddy of a given size; defined by the Bragg condition

$\kappa \quad$ Von Karman constant

$\lambda \quad$ acoustic wavelength

$\mu_{\max }$. maximum molecular absorption per wavelength

$\xi, \xi^{\dagger}, \eta, \eta^{\dagger} \quad$ coefficients of spectral density functions 
$\Phi(\mathrm{K}) \quad$ energy spectral density function for thermally induced turbulence

$\omega_{0} \quad$ angular molecular relaxation frequency

\section{GENERAL DESCRIPTION OF ATTENUATION PROBLEM}

A certain fraction of the power of an acoustic plane wave is lost by absorption mechanisms when the wave passes through a volume of air. The fractional loss in power is proportional to the distance that the wave travels through the medium

$$
\frac{\mathrm{dP}}{\mathbf{P}}=\alpha \mathrm{dr}
$$

where $\alpha$ is a proportionality constant. Figure 1 illustrates the general problem:

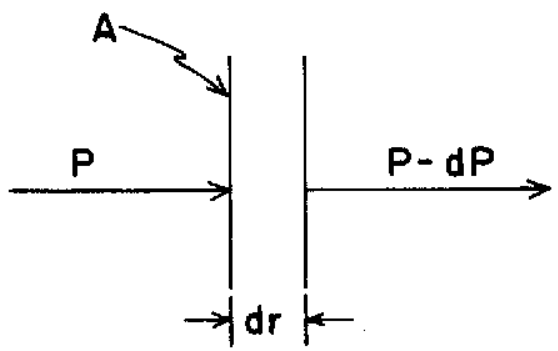

Figure 1.- Plane wave attenuation through a volume with
cross-sectional area A and thickness dr.

Consider a plane wave propagating vertically in the free atmosphere from an elevated source such as an airplane which is at some height $h$. (The fact that plane wave sources do not exist in nature will be supplanted with the usual plane wave approximations with the tacit assumption that spherical wave corrections to the derived results, which can be calculated in principle, are sufficiently small to be ignored to first order.) The acoustic power reaching the ground can be computed by integrating both sides of equation (1), with the following result:

$$
\mathrm{P}=\mathrm{P}_{\mathrm{o}} \mathrm{e}^{-\int_{0}^{\mathrm{h}} \alpha \mathrm{dr}}
$$

If the medium is homogeneous, $\alpha$ is independent of altitude and equation (2) reduces to

$$
\mathbf{P}=\mathbf{P}_{\mathrm{o}} \mathrm{e}^{-\alpha \mathrm{h}}
$$


The attenuation coefficient $\alpha$ depends in a complicated way on meteorological parameters and certain thermodynamic properties of the medium. A formula for this coefficient, derived from thermodynamic first principles in the nineteenth century, was found by Knudsen in 1931 (ref. 4) to be at least an order of magnitude too small to account for the attenuation observed experimentally in the audio range. Such observations led Kneser (ref. 2) to propose an additional attenuation mechanism involving the conversion of acoustic energy into the internal vibrational energy of the medium's constituent molecules. Thus, the absorption coefficient has been expressed as follows:

$$
\alpha=\alpha_{\mathrm{c}}+\alpha_{\mathrm{m}}
$$

where, for a given frequency, the classical absorption coefficient $\alpha_{\mathrm{c}}$ depends primarily on thermodynamic parameters and the molecular absorption coefficient $\alpha_{\mathrm{m}}$ depends on the concentration of impurity molecules such as $\mathrm{H}_{2} \mathrm{O}$.

The theory of classical plus molecular absorption has been shown to be in general agreement with experimental results in the audio range for measurements made under controlled laboratory conditions (refs. 3 and 12) but a number of measurements made out of doors have revealed the existence of an additional source of attenuation. (See refs. 5 to 11 and 13 to 16 .) That is, it appears that for outdoor measurements, equation (4) must be modified to include a third term:

$$
\alpha=\alpha_{\mathrm{c}}+\alpha_{\mathrm{m}}+\alpha_{\mathrm{s}}
$$

This additional attenuation (generally called the excess attenuation) has not been well understood. There is no universal agreement even on the mechanism for the phenomenon, since several possibilities exist, including refraction, interactions with suspended particulate matter or fog, scattering induced by turbulent density fluctuations, and certain anomalous vibrational relaxation processes yet to be defined.

A brief review of some experimental data is presented in the next section. Such a review will be helpful in determining the most likely candidate for the excess attenuation mechanism and will serve as an empirical guide for the formulation of an excess attenuation theory.

\section{EMPIRICAL DESCRIPTION OF EXCESS ATTENUATION}

Observations on the magnitude of the excess attenuation coefficient as well as its dependence on source altitude, frequency, and wind-speed gradients are discussed separately. 


\section{Magnitude}

There have been quite a wide range of magnitudes reported for the excess attenuation. In an experiment discussed in reference 17, Sieg measured the excess attenuation of a sound wave propagating from a source $12 \mathrm{~m}$ above the ground to a receiver near the ground and recorded values which were typically from 1 to $6 \mathrm{~dB}$ per $100 \mathrm{~m}$. However, since the receiver was located near the ground in these measurements, it is likely that ground effects may have interfered with the measurement. It is therefore likely that the values reported by Sieg represent an upper limit on the magnitude of the excess attenuation.

Ingard and Oleson (ref. 16) attempted to minimize ground effects by reversing the experimental procedure of Sieg. A source was mounted near the ground and a series of elevated receivers were arranged in a straight line oriented at an angle of about $1^{\circ}$ with respect to the horizontal. Although an excess attenuation was consistently observed, typical magnitudes were smaller than those reported by Sieg.

Outdoor attenuation measurements conducted by Delsasso and Leonard (ref. 13) were consistently found to exceed laboratory measurements by 20 percent to 80 percent. The experiment was conducted over a horizontal propagation path extending across a valley in the mountains of California where the source and receiver were at an altitude of approximately $3 \mathrm{~km}$ and separated by a distance of about $2 \mathrm{~km}$. Because the path length was so long, the results of this measurement probably only represent the average of extreme values for the excess attenuation cocfficient along the path. Thus, it is reasonable to expect a wider range in the magnitude of the excess attenuation coefficient than that reported in reference 13. Furthermore, if the excess attenuation is due primarily to turbulence, then the results in reference 13 probably underestimate the excess attenuation for the case of vertical propagation paths, over which meteorological gradients are much larger than those for horizontal propagation. The fact that turbulence levels decrease with increasing altitude also suggests that the results of this high-altitude experiment might represent a lower limit on typical excess attenuation magnitudes.

Beran et al. (refs. 5 and 11) studied the excess attenuation for a vertical propagation path by using a fixed receiver on the ground and a sound source mounted in a descending glider. Their results varied in magnitude from 0.01 to over 10 times greater than the classical plus molecular absorption. In a similar experiment in which a propeller-driven aircraft was used as a source, Benson et al. (refs. 7 to 10) reported an average excess attenuation of about the same order of magnitude as the classical plus molecular absorption.

Much of the available data on the excess attenuation have been supplied by the aircraft industry in the form of reported discrepancies between attenuation values measured in the field and published standard absorption values which are based on the classical plus molecular absorption theory alone, augmented by laboratory measurements in still air. 
For example, Coles (ref. 14) has analyzed the results of about 250 aircraft flyover measurements conducted by. Rolls Royce over a period of several years. He concludes that the field data are substantially larger than theoretical predictions based on equation (4) and that on the average, the theoretical predictions must be increased by a factor of from one to two to bring them in line with the measured data.

Although the magnitude of the excess attenuation coefficient appears to fluctuate a great deal from measurement to measurement, it is clear from the experimental results reviewed here that, in general, the magnitude of the excess attenuation in outdoor sound propagation is of the order of the classical plus molecular absorption.

\section{Altitude Dependence}

Two of the studies mentioned report data on the variation of the excess attenuation with source altitude. The data in both reports indicate that the excess attenuation coefficient depends on source altitude.

It is reported in references 7 to 10 that the excess attenuation is more pronounced near the Earth's surface than at higher altitudes. Figure 2, taken from reference 8, illustrates the altitude dependence of the excess attenuation schematically. The slope of this curve at a given height represents the excess attenuation coefficient at that height. It decreases with increasing height to a distance $h_{o}$ from the ground and remains almost constant above that distance. The value of $h_{o}$ is given as approximately $200 \mathrm{~m}(600 \mathrm{ft})$; this implies that the excess attenuation mechanism is active mainly below this altitude.

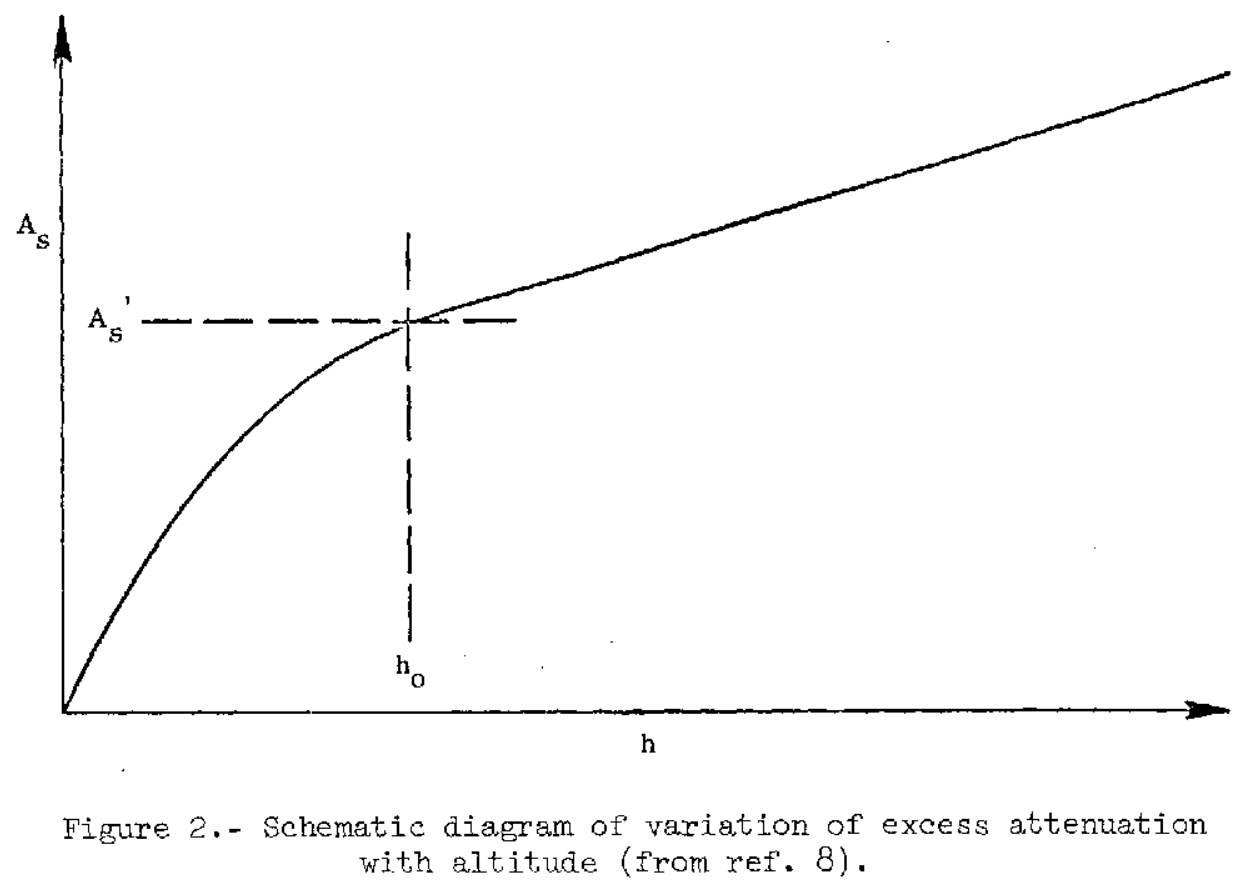


Although the dependence of the excess attenuation or source altitude was not discussed by Beran et al. (ref. 5), their data also indicate that the excess attenuation is greater at lower altitudes than at higher altitudes.

\section{Frequency Dependence}

Because the magnitude of the excess attenuation coefficient fluctuates so much from measurement to measurement even for a constant acoustic frequency, it is very difficult to specify the exact frequency dependence. Beran et al. (ref. 5) observed that the frequency dependence of the excess attenuation coefficient is weaker than the frequency dependence of the classical plus molecular absorption coefficient, which depends on frequency squared in the frequency range studied. Other observers also agree on the relatively weak frequency dependence of the excess attenuation.

In figure 3 , the average values of many measurements of the excess attenuation coefficient are plotted against frequency to the one-third power. The data when expressed

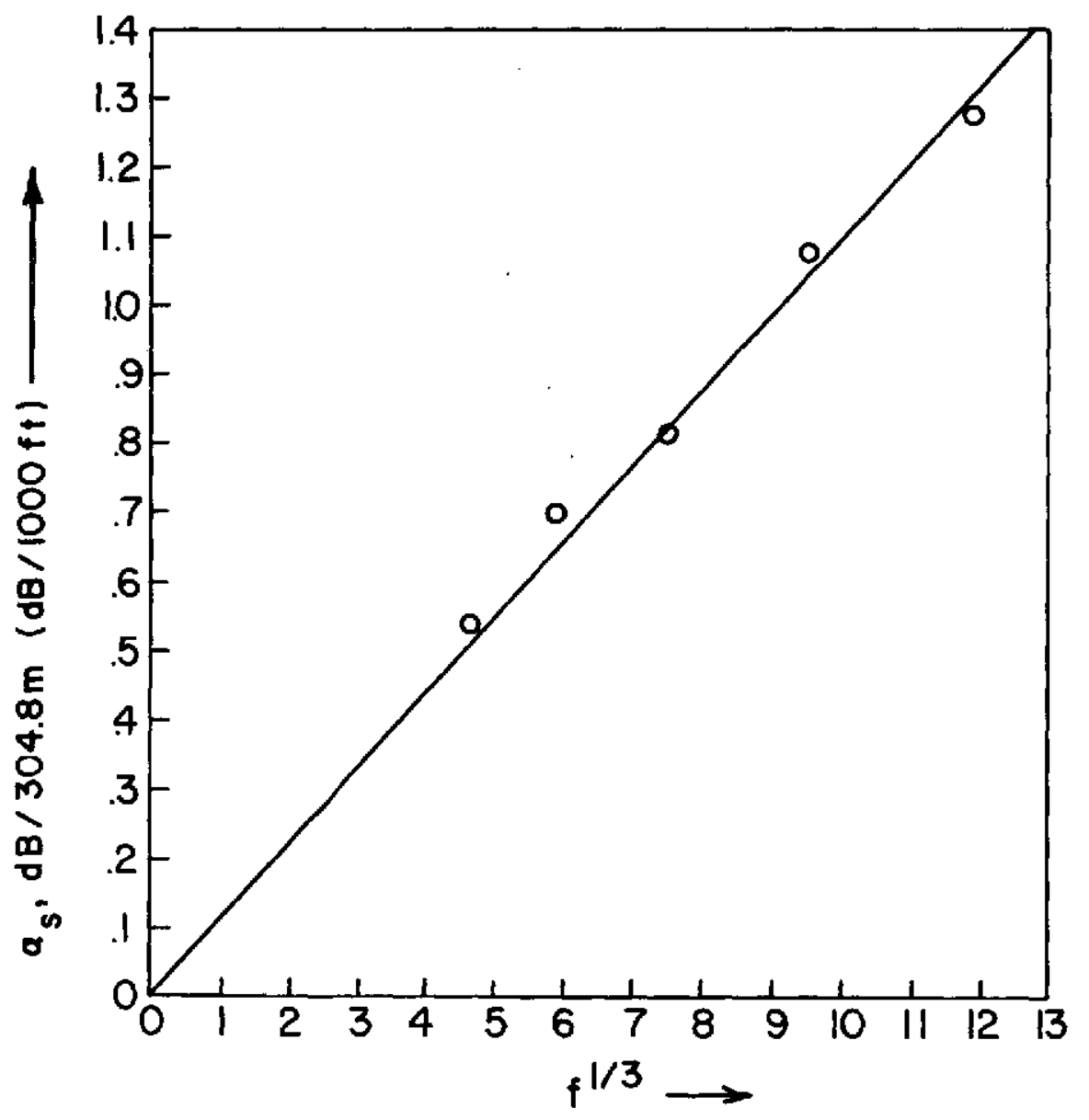

Figure 3.- Variation of excess attenuation with frequency to the one-third power (from ref. 8). $\alpha_{\mathrm{S}}=0.036 \mathrm{f}^{1 / 3} \mathrm{~dB} / 100 \mathrm{~m}$ or $0.1 \mathrm{lf} \mathrm{l} / 3 \mathrm{~dB} / 1000 \mathrm{ft}$. 
in units of $\mathrm{dB} / 1000 \mathrm{ft}$ fall on a straight line with a slope of 0.11 and lead to the following empirical formula for the excess attenuation coefficient:

$$
\alpha_{\mathrm{S}}=0.11 \mathrm{f}^{1 / 3} \mathrm{~dB} / 1000 \mathrm{ft}
$$

where $\mathrm{f}$ is the frequency in $\mathrm{Hz}$. These data are taken from reference 8 . An equivalent representation for this formula is

$$
\alpha_{\mathrm{S}}=0.036 \mathrm{f}^{1 / 3} \mathrm{~dB} / 100 \mathrm{~m}
$$

\section{Windspeed Gradients}

Ingard concludes in reference 19 that the excess attenuation depends on the "gustiness" of the wind. When the windspeed is irregular, the excess attenuation is generally higher than under calmer conditions.

A correlation between the excess attenuation and wind shear squared is reported in references 5,6 , and 11. It is concluded in these reports that inertial scattering, closely related to the energy input from wind shear, is the dominant excess attenuation mechanism but that other effects can play a significant role in the case of low shear.

Figure 4, taken from reference 16, illustrates that the excess attenuation is some nonlinear function of the mean windspeed. Since the magnitude of windspeed fluctuations may be expected to depend on the mean windspeed, this figure also tends to indicate that the excess attenuation increases with the irregularity (or "gustiness") of the mean flow.
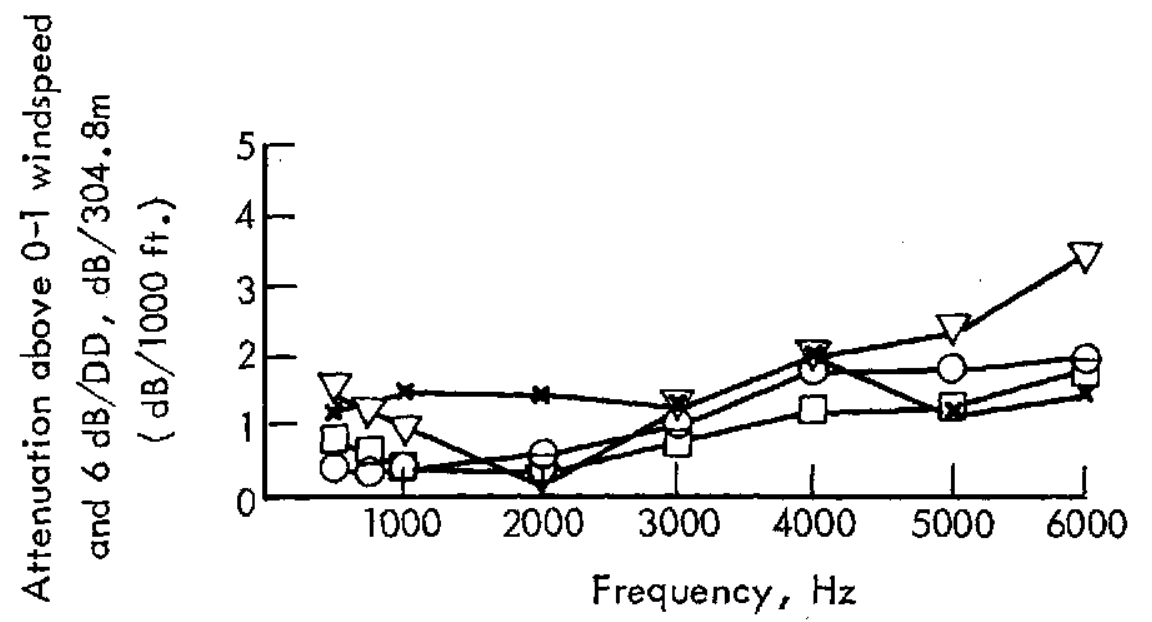

Mean windspeed

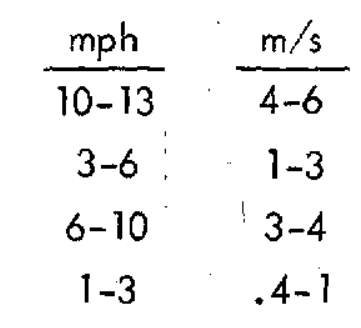

\footnotetext{
Figure 4. - Variation of scatter attenuation with frequency (after Ingard and Oleson). DD denotes double distance.
} 


\section{Effect of Time of Day}

In at least one report (ref. 5), there are data presented which reveal a correlation between the magnitude of the excess attenuation and the time of day during which the measurement was made. The relatively small number of data points and the limited range of meteorological conditions encountered in this experiment prohibit the results from being conclusive, but in nine series of measurements conducted over a 4-day period, the average excess attenuation at a given frequency was generally significantly greater in the early morning than in the late afternoon.

\section{Summary of Previous Experimental Results}

The results of the experiments reviewed are as follows:

(1) In general, the excess attenuation has as much effect on the propagation of outdoor sound as the classical plus molecular absorption.

(2) The instantaneous value of the excess attenuation coefficient undergoes rapid fluctuations over a wide range of magnitudes. The ratio of the excess attenuation coefficient to the classical plus molecular absorption coefficient can change by an order of magnitude in the period of a few minutes.

(3) The excess attenuation coefficient is a function of altitude; it is larger near the surface than at higher altitudes.

(4) The excess attenuation exhibits a weak frequency dependence. At least one observer has noted a cube root frequency dependence. In any case, the frequency dependence of the excess attenuation is much milder than the frequency dependence of the classical plus molecular absorption coefficient.

(5) There is a correlation between the excess attenuation and gradients in the windspeed. The more irregular the wind, the greater the excess attenuation; however, the excess attenuation is nonzero even when the wind shear is negligible.

(6) The data from at least one investigation indicates a correlation between the average magnitude of the excess attenuation and the time of day, larger values being observed in the early morning than in the late afternoon.

\section{POTENTIAL MECHANISMS FOR EXCESS ATTENUATION}

An excess attenuation can be the result of a number of effects, including refraction, ground attenuation, and interactions of the sound wave with turbulent density fluctuations or suspended particulate matter, including dust, solid pollutants, and fog droplets. Some molecular relaxation process, as yet not fully understood, may also be responsible for some aspects of the phenomenon. These possibilities are considered separately. 


\section{Refraction}

When the temperature of the atmosphere varies with height, the speed of sound also varies and results in curved sound ray paths instead of straight-line ray paths. During the day, the temperature usually decreases with increasing altitude. Sound rays which would tend to propagate horizontaliy in the absence of thermal gradients bend upward and this bending creates a shadow zone near the surface. At night, the thermal gradients reverse direction and the sound rays tend to bend downward in a phenomenon called focusing. Shadow zone formation and focusing also result from windspeed gradients. The windspeed usually increases with height so that sound rays propagating against the wind bend upward whereas sound rays propagating with the wind bend downward.

Under normal atmospheric conditions, vertical wind and temperature gradients are generally much larger than horizontal gradients. Refraction effects are therefore much more pronounced over horizontal propagation paths than over vertical propagation paths. It is reported in references 7 to 10 that the excess attenuation is virtually independent of propagation angle for propagation paths directed between $90^{\circ}$ and $14.5^{\circ}$ with respect to the horizontal. This implies that refraction effects are essentially negligible except for nearhorizontal propagation.

After performing a Snell's Law analysis based on sound velocities extracted from a measured vertical temperature profile, Beran et al. (ref. 5), concludes that in his excess attenuation measurements, refraction effects caused a reduction in received energy of less than 3 percent of the spherical spreading loss and was therefore too small to cause the observed excess attenuation.

Thomson and Shapiro (ref: 18) report the results of a calculation in which the atmosphere is divided into five layers, each of which is assigned a different set of meteorological conditions. The spectrum of perceived noise levels which would be received from a reference spectrum generated at an altitude of $1000 \mathrm{ft}(304.8 \mathrm{~m})$ is calculated. The calculation is repeated for a source height of $2000 \mathrm{ft}(609.6 \mathrm{~m})$. It is concluded in this report that the errors due to ray-path curvature are smaller than the normal uncertainty associated with precision field acoustic measurements, even for the most extreme meteorological profiles likely to be encountered in a vertical propagation experiment. The results of the studies reviewed here imply that for the case of nonhorizontal propagation paths, it is unlikely that refraction effects contribute substantially to the excess attenuation.

\section{Ground Attenuation}

Ground attenuation has the greatest effect on sound propagating horizontally and near the surface. The magnitude of the ground attenuation depends on the acoustic impedance of the terrain and, according to calculations discussed in reference 19, it is a maximum at frequencies for which the wavelength is on the order of one-half to two-thirds the 
height of the source above the ground. This result implies that in the audio range ground attenuation should make only a small contribution to the excess attenuation associated with an aircraft flyover. The fact that Delsasso and Leonard (ref. 13) observed a large excess attenuation over a wide mountain valley also suggests that ground effects do not contribute substantially to the excess attenuation, since in this experiment the propagation path was far above the ground.

\section{Effects of Dust}

Henley and Hoidale (ref. 20) have studied the effects of suspended particulate matter on sound propagation in the atmosphere and have concluded that although dust affects the dispersion, under normal atmospheric conditions the attenuation due to dust is negligible. This conclusion is supported by observations made in reference 5 , in which an appreciable excess attenuation was measured at a site remote from pollution sources and under general synoptic conditions which led the investigators to conclude that dust concentrations were not responsible for the observed excess attenuation.

Although suspended particulate matter in very high concentrations can have an appreciable effect on sound transmission (ref. 21), typical atmospheric dust concentrations are generally so small that it is unlikely that dust is responsible for the excess attenuation.

\section{Effects of Fog}

A discussion of some of the observed effects of fog on sound transmission in the atmosphere has been given by Ingard ( $r$ ef. 19). A number of experimental investigations are cited, the results of which indicate that sound is transmitted easily through fog. A theory to describe sound attenuation due to fog has been put forth by Cole and Dobbins (ref. 22) which predicts a relatively weak interaction under typical fog conditions in the atmosphere. Data reported by Harris (refs. 23 and 24) indicate that molecular attenuation for the case of sound propagating through saturated air is less than for the case of sound propagating through less humid air. Thus, there are conditions when the attenuation of sound can be less in fog than in clear air with a relatively reduced moisture content.

Tyndall (ref. 25) attributed the ease with which sound propagates through fog as a reflection of the relatively calm atmospheric conditions, devoid of strong wind and temperature gradients, which generally accompany fog. In any case, there have been a sufficiently large number of excess attenuation measurements made in the absence of fog to eliminate it as the primary cause of the excess attenuation. 


\section{Relaxation Processes}

Relaxation processes involving $\mathrm{O}_{2}$ vibrational resonances are primarily responsible for the molecular absorption. The possibility that other relaxation processes might be responsible for the excess attenuation is discussed in reference 15; however, the peculiar altitude and frequency dependence of the excess attenuation, the strong correlation with windspeed gradients, and the fact that a large excess attenuation is basically an outdoor phenomenon all argue against this possibility.

\section{Effects of Turbulence}

As a sound wave propagates through a turbulent medium, it interacts with turbulent density fluctuations in the medium. Local momentum fluctuations induced by eddy motion cause changes in the local pressure which radiate away as a scattered acoustic wave. This type of interaction is referred to as inertial scattering. Temperature fluctuations in the atmosphere and local changes in the acoustic index of refraction caused by the resulting density fluctuations are also responsible for scattering.

The effect of inertial and thermal scattering is to direct some of the incident acoustic energy away from the original propagation direction. There is no reduction in the total transmitted acoustic power but that power is diffused over a wider area, with the result that the received intensity along the original direction is diminished. There is no way for the receiver to distinguish between this effect and a bona-fide absorption loss.

Turbulence is related to wind shear and "gustiness" which have been observed to produce large increases in the excess attenuation (refs. 5, 6, 11, 16, and 19). The dependence on height above ground also suggests a boundary-layer type of mechanism.

The small effects observed and/or calculated for the other factors and the positive correlation between wind effects and the excess attenuation suggest that scattering due to turbulence is responsible for most of the excess attenuation measured out of doors over nonhorizontal propagation paths. The effects of turbulence on sound propagation are considered in more detail in the remainder of this report.

\section{BRIEF REVIEW OF EARLIER THEORIES, FOUNDATION, AND APPROACH}

Historically, the theory of sound propagation in a turbulent medium has advanced along two parallel lines of development. In the first approach, the atmosphere is assumed to consist of an ensemble of vortical eddies; for this medium the problem of acoustic scattering reduces to an extension of single vortex theory to the case of multiple scattering among many vortical eddies. This approach is used by Lindsay (ref. 26) and Müller 
and Matschat (ref. 27) among others. In the second approach, turbulence is described in terms of stochastic processes in the atmosphere in which such ensemble characteristics as means and correlation coefficients play a central role. Among those workers who have used this approach are Obukov (refs. 28 and 29), Batchelor (ref. 30), Kraichnan (ref. 31), Pekeris (ref. 32), Blokhintzev (refs. 33 and 34), Tatarski (ref. 35), Kallistratova (ref. 36), Kallistratova and Tatarski (ref. 37), Monin (ref. 38), and Lighthill (ref. 39).

The single vortex extension technique has certain disadvantages associated with it which have retarded its application to the solution of "real world" problems. For example, because of the computational complexities involved in a theory of multiple vortex scattering, certain unrealistic assumptions must of ten be made about the uniformity of the eddy sizes. The mean vortex radius, which is difficult to determine experimentally, appears as a fundamental parameter in the theory. Furthermore, thermal scattering is neglected; only the effects of velocity perturbations resulting in inertial scattering are considered in the multiple scattering method.

Müller and Matschat (ref. 27) applied the single vortex extension method to derive a formula for the excess attenuation coefficient which reduces to the following form for the case of acoustic wavelengths that are small compared with the turbulence scale:

$$
\alpha_{s}=20\left(1-\frac{2}{\pi}\right)(\log e) \frac{\overline{\Delta v^{2}}}{c^{2}} L^{2}
$$

or

$$
\alpha_{\mathrm{S}}=3.156 \frac{\overline{\Delta \mathrm{v}^{2}}}{\mathrm{c}^{2}} \mathrm{Lk}^{2}
$$

This result is valid, according to the Müller-Matschat theory, when the sound is propagat ing in a direction perpendicular to the vortex axes. Here, $L$ is the mean vortex radius, $\overline{\Delta v^{2}}$ is the mean-square turbulence velocity fluctuation, $\mathrm{c}$ is the speed of sound, and $\mathrm{k}$ is the acoustic wave number. (In the notation of ref. $27, \overline{\Delta \mathrm{v}^{2}} / \mathrm{c}^{2}$ is called $\overline{\mathrm{M}}^{2}$, the mean turbulence Mach number squared, and the symbol $r_{a}$ is used in place of $L$.)

Lighthill (ref. 39) used a more representative model for atmospheric turbulence in which the assumption of a uniform eddy size is replaced with the concept of a spectrum of eddy sizes. Starting from his general theory of aerodynamically generated sound (ref. 40), he derived the following formula for the coefficient of attenuation due to scattering for the case of isotropic turbulence:

$$
\alpha_{S}=2 \frac{\overline{\Delta v^{2}}}{c^{2}} \mathrm{Lk} \mathrm{k}^{2}
$$


where $L$ has units of length and is a measure of the "width" of the main energy-bearing eddies in the direction of propagation. (In ref. 40, $L$ is called $L_{1}, c$ is called a, and $\alpha_{\mathrm{S}}$ is called $\beta$. The attenuation is expressed in terms of wavelength instead of wave number in the original reference.) Batchelor (ref. 30) derived this same result by using a different approach.

Chernov (ref. 41) considered the interaction of sound waves with thermally induced refractive index fluctuations. Assuming an exponential form for the spatial correlation coefficient describing these fluctuations, he derived the following result for the excess attenuation coefficient:

$$
\alpha_{\mathrm{S}}=\frac{\overline{8 \mu^{2}} \mathrm{k}^{4} \mathrm{~L}^{3}}{1+4 \mathrm{k}^{2} \mathrm{~L}^{2}}
$$

where $\overline{\mu^{2}}$ is the mean-square departure of the refractive index from its mean value. It is shown by Buell (ref. 42) that $\overline{\mu^{2}} \approx \frac{\Delta T^{2}}{4 T^{2}}$, where $T$ is the absolute temperature and $\overline{\Delta \mathrm{T}^{2}}$ is the mean-square temperature fluctuation. If the wavelength of the incident acoustic wave is small compared with the turbulence scale $L$, then equation (9) reduces to

$$
\alpha_{s}=2\left(\frac{\Delta \mathrm{T}^{2}}{4 \mathrm{~T}^{2}}\right) \mathrm{Lk}^{2}
$$

which is of the same functional form as the formula describing the coefficient of attenuation due to inertial scattering (eqs. (7) and (8)).

The early theories which have been described briefly here all predict the same general features for the coefficient of attenuation due to scattering; namely, it is a function of turbulent velocity or temperature fluctuations in the medium and it is proportional to a length scale which is in some sense a measure of the size of the turbulent eddies. Both of these results are in harmony with intuitive expectations and the first agrees in general with experimental observations. However, the square-law frequency dependence predicted by these earlier theories is not confirmed experimentally and the observed excess attenuation is much smaller than that predicted by equations (7), (8), and (10). For example, Lighthill (ref. 39) compared his theoretical result (eq. (8)) with the experimental results of Kneser, Knudsen, and others and found that it predicted an excess attenuation per wavelength which would exceed the maximum measured molecular attenuation per wavelength (attained at a frequency which depends on the moisture content, among other things) when the mean-square turbulence velocity fluctuations exceed the rather modest value of $1.7 \mathrm{~m} / \mathrm{sec}$, even if the turbulence scale $\mathrm{L}$ were as small as one wavelength. The turbulence scale is typically at least an order of magnitude larger than one wavelength for audio 
frequencies in the atmosphere. Therefore, the theory of Lighthill (and also those of Batchelor, Müller and Matschat, and Chernov) predicts excess attenuation magnitudes which are much larger than the measured values. As a further example, Ingard and Weiner (ref. 43) compared the results of field measurements of the excess attenuation with the Lighthill theory and noted that the observed excess attenuation was much smaller than this theory predicts. It was also noted that the strong frequency dependence predicted in the Lighthill theory was not confirmed experimentally. The purpose of the present study is to explain the experimental observations noted here and earlier in this report.

Early advances in the statistical theory of turbulence eventually led to a scattering cross-section formula developed by Tatarski (ref. 35) and further refined by Kallistratova and Tatarski (ref. 37):

$$
\frac{\mathrm{d} \sigma(\theta)}{\mathrm{d} \Omega}=2 \pi \mathrm{k}^{4} \mathrm{~V} \cos ^{2} \theta\left[\frac{\mathrm{E}(\mathrm{K})}{\mathrm{c}^{2}} \cos ^{2} \frac{\theta}{2}+\frac{\Phi(\mathrm{K})}{4 \mathrm{~T}^{2}}\right]
$$

This same result was derived independently by Monin (ref. 38). - The differential scattering cross section describes the fraction of the incident acoustic power scattered into a solid angle $d \Omega$ by a volume $V$ of turbulence, where $E(K)$ and $\Phi(K)$ are the spectral density functions describing velocity and temperature fluctuations, respectively. The spectral density functions depend on $K$, the turbulence wave number, where $K=2 \pi / \ell$ and $\ell$ is an eddy size parameter. The scattering angle $\theta$ is with respect to the direction of propagation; $\mathrm{c}$ and $\mathrm{T}$ are the speed of sound and the mean absolute temperature, respectively. Equation (11) has been shown to agree satisfactorily with experiment (see refs. 36 and 44 for cross-section measurements) and the calculations which follow are based on this representation for the scattering cross section.

\section{PRESENT DEVELOPMENT}

\section{Turbulence Model}

Turbulence is very difficult to define succinctly except by its contrast with laminar flow and there is no universally accepted model to describe quantitatively the details of a turbulent medium. For the purposes of this study, it is assumed that turbulence in the lower atmosphere (the first few hundreds of meters) has the following properties:

(1) The atmosphere is assumed to consist of an ensemble of turbulent eddies, each of which is characterized by a length scale $\ell$ which in some sense represents the size of the eddy. 
(2) There exists a bounded spectrum of eddy sizes ranging from a minimum value $\ell_{0}$ called the inner scale of turbulence, to some maximum outer scale size. The inner scale depends on viscosity and the energy dissipation rate of the medium and is typically on the order of a few millimeters in the lower atmosphere. The outer scale depends on the synoptic climatology and also on the physical features of the local terrain and may range in magnitude from tens to several hundreds of meters.

(3) The spectrum of eddy sizes is continuous between the inner and outer scale; that is, there are no "forbidden" eddy sizes within the spectrum.

(4) Each eddy is classified as either a "small-scale" eddy or a "large-scale" eddy. (The large-scale eddies are also called "outer-scale" eddies in this report.) The smallscale eddies are basically homogeneous and isotropic whereas the large-scale eddies generally are not.

(5) An eddy is called homogeneous if the mean values of the random meteorological fields which characterize that eddy are constant and if the correlation functions describing those fields are insensitive to displacement within the eddy. A homogeneous eddy is called isotropic if the correlation functions are also insensitive to rotations and mirror reflections within the eddy.

At the onset of turbulence, energy is introduced into the medium through the largest eddies by the effects of wind shear and convective heating. The large-scale eddies form and transfer energy to smaller eddies. Energy continues to cascade in this manner to smaller and smaller eddies until the inner scale is reached, where viscous effects cause the energy to be dissipated.

The energy of a turbulence spectrum is described mathematically with an energy spectral density function, generally expressed in terms of the so-called turbulence wave number $K$, which can be related to the eddy size in the same way that the wave number of an acoustic plane wave is related to its wavelength. This interpretation of the turbulence wave number is somewhat intuitive but a more rigorous discussion of the concept can be found in reference 39 .

The form of the energy spectral density function cannot be uniquely determined for eddies on the order of the outer scale (small $K$ values) since the behavior of these eddies depends on the local mechanisms responsible for introducing energy into the turbulence spectrum and is therefore influenced by such factors as local climatology and terrain roughness. For eddies which are small compared with the outer scale and large compared with the inner scale, the spectral density function has been shown theoretically and experimentally to have a $\mathrm{K}^{-11 / 3}$ dependence. This range of wave numbers is called the inertial subrange. 
If the Reynolds number is large, as is generally the case in the atmosphere, the inertial subrange is fairly extensive. (See ref. 35.) For example, measurements reported in reference 45 reveal an inertial subrange covering five orders of magnitude in $\mathrm{K}$ space. For $\mathrm{K} \approx 2 \pi / l_{0}$, the spectral density functions are known to fall off faster than $\mathrm{K}^{-11 / 3}$ but because the small-scale eddies contribute so little to the total energy of the spectrum, the assumption of a $\mathrm{K}^{-11 / 3}$ dependence in this, the so-called dissipation range, is not a bad approximation, at least as far as the sound propagation problem is concerned. In any case, it has been shown that eddies which are small compared with half a wavelength of the incident acoustic plane wave do not affect the propagation of sound significantly. (See ref. 39.) Therefore, the precise functional form for the spectral density functions for very large $\mathrm{K}$ is not important in this investigation. A typical spectral density function is represented schematically in figure 5 .

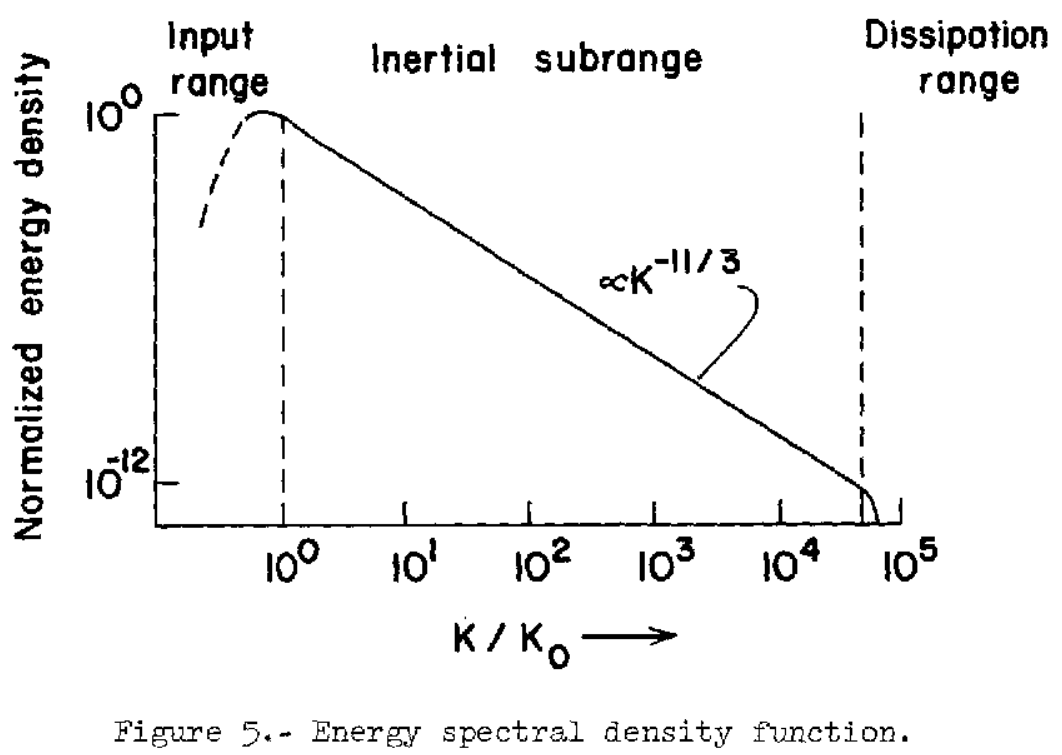

Theory

Consider a plane wave with wave number $\vec{k}$ incident on a turbulent eddy as in figure 6. Some of the incident energy is scattered at an angle $\theta$. The scattered wave

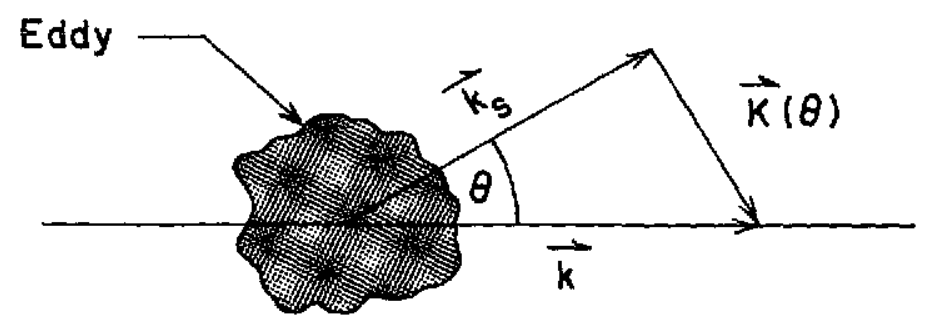

Figure 6.- Scattering of sound by turbulence. 
has a wave number $\overrightarrow{\mathrm{k}_{\mathrm{S}}}$ where, Doppler shifts being neglected, $\quad\left|\overrightarrow{\mathrm{k}_{\mathrm{S}}}\right|=|\overrightarrow{\mathrm{k}}|$. It is customary to characterize a turbulent eddy by a vector $\overrightarrow{\mathrm{K}}(\theta)$ which represents the vector difference between the incident and scattered wave number vectors:

$$
\overrightarrow{\mathbf{K}}(\theta)=\overrightarrow{\mathbf{k}}-\overrightarrow{\mathbf{k}}_{\mathbf{S}}
$$

Since $\left|\overrightarrow{\mathrm{k}}_{\mathrm{S}}\right|=|\overrightarrow{\mathrm{k}}|$, equation (12) can be shown to have the following form:

$$
\mathrm{K}=2 \mathrm{k} \sin \frac{\theta}{2}
$$

If the customary assumption is made that $\mathrm{K}$ can be expressed in terms of an eddy size $\ell$ in the same way that an acoustic wave number is expressed in terms of wavelength, then equation (13) can be rewritten as

$$
\lambda=29 \sin \frac{\theta}{2}
$$

Equation (14) describes the angle through which an incident acoustic plane wave with wavelength $\lambda$ will be scattered when it encounters an eddy characterized by a scale length $\ell$. This is just a statement of the familiar Bragg condition which describes $\mathrm{X}$-ray diffraction in crystals. Thus, the scattering of sound by turbulence can be modeled as a Bragg diffraction phenomenon in which the turbulence eddies form diffraction gratings in the atmosphere which are responsible for scattering the incident sound just as the atoms of a crystal form the "diffraction gratings" which cause X-rays to scatter in a crystal. Because there is a continuous spectrum of eddy sizes in a turbulent medium, the atmosphere is really a continuous superposition of gratings, each with a different period $\ell$ and each responsible for scattering a given wavelength in a particular direction, according to equation (14).

Equation (14) was obtained in a very general way, with no particular constraints placed on the scattering eddy. However, it is reasonable to suspect the range of validity of this result. The "atmospheric crystal" model might be expected to hold in the case of small-scale eddies which are sufficiently homogeneous to have the same general effect on an incident acoustic plane wave as crystalline atoms have on an incident electromagnetic plane wave, but the crystal model should break down for the outer scale eddies, which are patently inhomogeneous. Hence, although the Bragg condition may be valid for smallscale eddies, it is unlikely that it is valid near the outer scale.

The remainder of this section is divided into two parts. In the first part, a homogeneous and isotropic medium will be considered in which the Bragg condition (eq. (14)) applies for all eddies, including those on the order of the outer scale. In the second part, 
a different medium is considered in which the conventional Bragg condition represents only a first approximation to the scattering process.

(1) Homogeneous and Isotropic Turbulence

The scattering cross section (eq. (11)) describes the fraction of power scattered into the solid angle $d \Omega$ in the $\theta$ direction for a given incident intensity $I$ :

$$
\frac{\mathrm{dP}}{\mathrm{d} \Omega}=\mathrm{I} \frac{\mathrm{d} \sigma}{\mathrm{d} \Omega}
$$

Insert equation (11) into equation (15) to obtain

$$
\mathrm{dP}=2 \pi \mathrm{k}^{4} \mathrm{IV} \cos ^{2} \theta\left[\frac{\mathrm{E}(\mathrm{K})}{\mathrm{c}^{2}} \cos ^{2} \frac{\theta}{2}+\frac{\Phi(\mathrm{K})}{4 \mathrm{~T}^{2}}\right] \mathrm{d} \Omega
$$

Figure 1 illustrates a plane wave incident on a volume of air with cross-sectional area $\mathrm{A}$ and thickness $\mathrm{dr}$. If the air contains turbulence, then the reduction in power suffered by the incident sound wave due to scattering is described by equation (16) where $\mathrm{V}=\mathrm{Adr}$. The product of the incident intensity and the cross-sectional area of the turbulent region is just the power $P$ incident upon the volume $A d r$. Thus, equation (16) becomes

$$
\mathrm{dP}=2 \pi \mathrm{k}^{4} \mathrm{P} \cos ^{2} \theta\left[\frac{\mathrm{E}(\mathrm{K})}{\mathrm{c}^{2}} \cos ^{2} \frac{\theta}{2}+\frac{\Phi(\mathrm{K})}{4 \mathrm{~T}^{2}}\right] \mathrm{d} \Omega \mathrm{dr}
$$

Because of the azimuthal symmetry of the scattering process, the differential solid angle $\mathrm{d} \Omega$ can be written as $2 \pi \sin \theta \mathrm{d} \theta$ and equation (17) becomes

$$
\frac{\mathrm{dP}}{\overline{\mathrm{P}}}=4 \pi^{2} \mathrm{k}^{4} \cos ^{2} \theta\left[\frac{\mathrm{E}(\mathrm{K})}{\mathrm{c}^{2}} \cos ^{2} \frac{\theta}{2}+\frac{\Phi(\mathrm{K})}{4 \mathrm{~T}^{2}}\right] \sin \theta \mathrm{d} \theta \mathrm{dr}
$$

Note the similarity between this equation and equation (1), which describes the general absorption problem. If a plane wave is propagating from a height $h$, where its power is $\mathrm{P}_{0}$, to a receiver on the ground, then the power reaching the receiver can be computed, absorption effects for the moment being neglected, by integrating both sides of equation (18 with the following result:

$$
\mathrm{P}=\mathrm{P}_{\mathrm{o}} \exp \left(-\int_{0}^{\mathrm{h}}\left\{\int_{0}^{\pi} 4 \pi^{2} \mathrm{k}^{4} \cos ^{2} \theta\left[\frac{\mathrm{E}(\mathrm{K})}{\mathrm{c}^{2}} \cos ^{2} \frac{\theta}{2}+\frac{\Phi(K)}{4 \mathrm{~T}^{2}}\right] \sin \theta \mathrm{d} \theta\right\} \mathrm{dr}\right)
$$


By comparing equation (19) with equation (2), it is clear that the coefficient of attenuation due to scattering is as follows:

$$
\alpha_{s}=\int_{0}^{\pi} 4 \pi^{2} k^{4} \cos 2 \theta\left[\frac{E(K)}{c^{2}} \cos ^{2} \frac{\theta}{2}+\frac{\Phi(K)}{4 T^{2}}\right] \sin \theta d \theta
$$

where $K$ is a function of $\theta$. In an idealized medium, for which the Bragg condition applies even to the outer scale eddies, $K$ and $\theta$ are related through equation (13).

It has already been stated that the small $\mathrm{K}$ functional forms of the spectral density functions $E(K)$ and $\Phi(K)$ are not universal, depending as they do on local ter rain conditions and synoptic climatology. It is therefore impossible to arrive at a completely general formula for the coefficient of attenuation due to scattering by solving the integral in equation (20); the integrand is slightly different for every special case. However, for a turbulent medium such as the lower atmosphere, with an inertial subrange which is very large compared with the range of large-scale eddies, it will be shown that to first order, the coefficient of attenuation due to scattering is independent of the precise functional form of the spectral density functions near the origin. It is therefore possible to evaluate the integral in equation (20) without knowing the details of the spectral density functions for small K. To see this, express the spectral density functions in the following general form:

$$
\begin{aligned}
& \mathrm{E}(\mathrm{K})= \begin{cases}\xi \mathrm{K}^{\mathrm{n}} & \left(\mathrm{K}>\mathrm{K}_{\mathrm{O}}\right) \\
\xi^{\prime} \mathrm{K}^{\mathrm{m}} & \left(\mathrm{K} \leqq \mathrm{K}_{\mathrm{O}}\right)\end{cases} \\
& \Phi(\mathrm{K})= \begin{cases}\eta \mathrm{K}^{\mathrm{n}} & \left(\mathrm{K}>\mathrm{K}_{\mathrm{O}}\right) \\
\eta^{\prime} \mathrm{K}^{\mathrm{m}} & \left(\mathrm{K} \leqq \mathrm{K}_{\mathrm{O}}\right)\end{cases}
\end{aligned}
$$

Here, $K_{0}$. represents some outer scale turbulence wave number which marks the threshold between the inertial subrange and the range of large-scale eddies, as in figure 5. For $\mathrm{K}>\mathrm{K}_{\mathrm{O}}$, the spectral density functions are understood and the unprimed coefficients and $n$ are therefore known. The primed coefficients and $m$ describe the spectral density functions near the origin. Their values depend on the local conditions responsible for introducing energy into the turbulence spectrum and are therefore not universal and are generally unknown.

The integral in equation (20) is evaluated in appendix A by using the spectral density functions of equations (21). By assuming that the outer scale is large compared with a 
half wavelength and imposing some very mild constraints on $m$ and $n$, the following first-order result is obtained:

$$
\alpha_{\mathrm{S}}=\frac{4 \pi^{2} \mathrm{k}^{2} \mathrm{~K}_{\mathrm{O}}^{\mathrm{n}+2}}{-(\mathrm{n}+2)}\left(\frac{\xi}{\mathrm{c}^{2}}+\frac{\eta}{4 \mathrm{~T}^{2}}\right)
$$

Note that neither $m$ nor either of the primed coefficients of equations (21) appear in this result. Thus, the excess attenuation coefficient depends only on the large $K$ form of the spectral density functions.

Tatarski (ref. 35) gives the following formulas for the spectral density functions in the inertial subrange:

$$
\begin{aligned}
& E(K)=\frac{11 \Gamma\left(\frac{8}{3}\right) \sin \frac{\pi}{3} C_{V}^{2}}{24 \pi^{2}} K^{-11 / 3} \\
& \Phi(K)=\frac{\Gamma\left(\frac{8}{3}\right) \sin \frac{\pi}{3} C_{T^{2}}^{2}}{4 \pi^{2}} K^{-11 / 3}
\end{aligned}
$$

where $\mathrm{C}_{\mathrm{V}}$ and $\mathrm{C}_{\mathrm{T}}$ are called the structure constants for wind shear and thermally induced turbulence, respectively. The constant $\mathrm{n}$ and the coefficients $\xi$ and $\eta$ can be determined by comparing equations (23) with equations (21a) and (21c):

$$
\begin{aligned}
& \xi=\frac{11 \Gamma\left(\frac{8}{3}\right) \sin \frac{\pi}{3} \mathrm{C}_{\mathrm{V}}^{2}}{24 \pi^{2}} \\
& \eta=\frac{\Gamma\left(\frac{8}{3}\right) \sin \frac{\pi}{3} \mathrm{C}_{\mathrm{T}}^{2}}{4 \pi^{2}} \\
& \mathrm{n}=-\frac{11}{3}
\end{aligned}
$$


When equations (24) are inserted into equation (22), the result is

$$
\alpha_{S}=\left[1.1 \Gamma\left(\frac{8}{3}\right) \sin \frac{\pi}{3}\right] \mathrm{k}^{2} \mathrm{~K}_{\mathrm{o}}^{-5 / 3}\left(\frac{\mathrm{C}_{\mathrm{V}}^{2}}{\mathrm{c}^{2}}+\frac{3}{22} \frac{\mathrm{C}_{\mathrm{T}}{ }^{2}}{\mathrm{~T}^{2}}\right)
$$

or, after combining the numerical constants,

$$
\alpha_{\mathrm{S}}=1.442 \mathrm{k}^{2} \mathrm{~K}_{\mathrm{o}}^{-5 / 3}\left(\frac{\mathrm{C}_{\mathrm{V}}{ }^{2}}{\mathrm{c}^{2}}+0.136 \frac{\mathrm{C}_{\mathrm{T}}{ }^{2}}{\mathrm{~T}^{2}}\right)
$$

Equations (25) represent a first-order formula for the coefficient of attenuation due to scattering in a homogeneous, isotropic medium with a low mean flow speed for the case of audio wavelengths which are small compared with the outer scale of turbulence. This result does not depend on the details of the spectral density functions for small $\kappa$, subject to some very mild conditions which can always be expected to be met for a physically realizable turbulence spectrum. (See appendix A.)

One can compare the present result with the results of earlier investigations by transforming equations (25) from a structure constant representation to a representation in terms of wind and temperature fluctuations. Applying a method used by Strohbehn (ref. 46) to the case of a homogeneous and isotropic spectrum of turbulence for which equations (21) describe the spectral density functions, the structure constants $\mathrm{C}_{\mathrm{V}}$ and $\mathrm{C}_{\mathbf{T}}$ can be expressed in terms of mean-square fluctuations in the windspeed and the temperature, respectively. This calculation is performed in appendix B with the following results:

$$
\begin{aligned}
& \mathrm{C}_{\mathrm{V}}{ }^{2}=\frac{4 \pi \mathrm{K}_{\mathrm{o}}^{2 / 3} \overline{\Delta \mathrm{v}^{2}}}{11 \Gamma\left(\frac{8}{3}\right) \sin \frac{\pi}{3}} \\
& \mathrm{C}_{\mathrm{T}}{ }^{2}=\frac{2 \pi \mathrm{K}_{\mathrm{O}}^{2 / 3} \overline{\Delta \mathrm{T}^{2}}}{3 \Gamma\left(\frac{8}{3}\right) \sin \frac{\pi}{3}}
\end{aligned}
$$


Insert equations (26) into equations (25) to obtain

$$
\alpha_{s}=0.2 L_{o} k^{2}\left(\frac{\Delta v^{2}}{c^{2}}+\frac{\Delta T^{2}}{4 T^{2}}\right)
$$

The difference between this result and the earlier results of Lighthill (eq. (8)) and Chernov (eq. (10)) is that the numerical coefficient in equation (27) is an order of magnitude smaller than the corresponding numerical coefficient in these earlier results. This is consistent with the experimental results of Ingard and Wiener (ref. 43) whose field measurements of the excess attenuation were much smaller than the Lighthill theory predicts.

Ingard and Wiener also reported a milder frequency dependence than that predicted by either equation (27) or the earlier excess attenuation theories. This observation, which is also reported in references 5 to 11,16 , and 19 , cannot be explained with the simple scattering model considered. It will be shown in the next section that the experimentally observed frequency dependence of the excess attenuation can be explained if the Bragg condition (eq. (13)) is replaced with a more general expression relating the scattering angle and eddy size.

(2) Extension of Model to Real Atmosphere

It has been assumed previously that the scattering of acoustic plane waves by atmospheric turbulence is described by equation (13), the Bragg condition. The simple model upon which this assumption is based is not likely to be entirely valid for the real atmosphere, where the inhomogeneity and anisotropy of the outer scale eddies, fluctuations in the size of the eddies, and other departures from the simple model considered above influence the scattering process. It is appropriate to ask how the vector representing a scattered wave in the real atmosphere would differ from a scattered wave vector in the ideal medium considered previously. The scattering process in the real atmosphere may be represented by figure 7 .

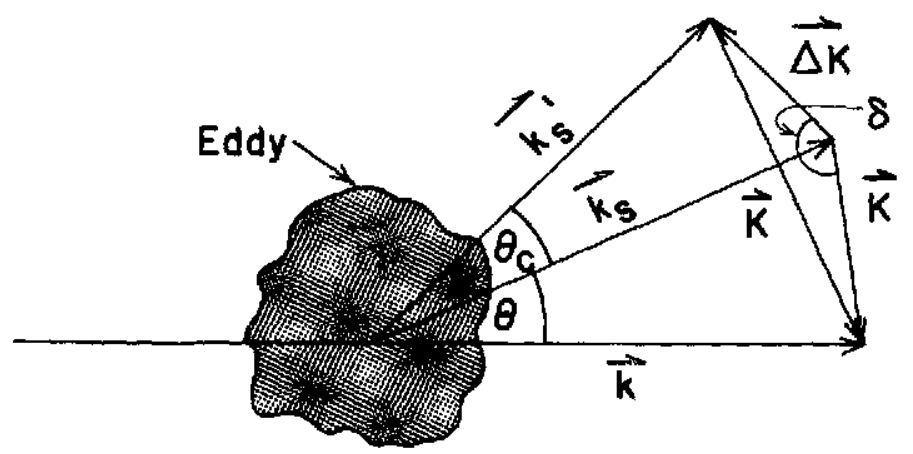

Figure 7.- Scattering by large scale eddy. 
In figure $7, \vec{k}$ is the incident wave number vector, $\overrightarrow{\mathrm{k}}_{\mathrm{S}}$, is the wave number vector which would describe the scattered wave if equation (13) were valid, and $\vec{k}_{\mathbf{S}}$ is the actual scattered wave number vector. The angle $\theta_{\mathrm{c}}$ then represents the average difference between the actual scattering direction and the scattering direction predicted by the conventional Bragg diffraction model considered earlier. As can be seen in the figure, the vectors $\overrightarrow{\mathrm{K}}, \overrightarrow{\mathrm{K}}$, and $\Delta \overrightarrow{\mathrm{K}}$ are related to the acoustic wave number vectors as follows:

$$
\begin{aligned}
& \overrightarrow{\mathrm{K}}=\overrightarrow{\mathrm{k}}-\overrightarrow{\mathrm{k}_{\mathrm{S}}} \\
& \overrightarrow{\mathrm{K}}^{\prime}=\overrightarrow{\mathrm{K}}-\overrightarrow{\Delta \mathrm{K}} \\
& \overrightarrow{\Delta \mathrm{K}}=\overrightarrow{\mathrm{k}}_{\mathrm{S}}-\overrightarrow{\mathrm{k}}_{\mathrm{s}}
\end{aligned}
$$

Doppler effects being neglected, $|\overrightarrow{\mathrm{k}}|=\left|\overrightarrow{\mathrm{k}}_{\mathrm{S}}\right|=\left|\overrightarrow{\mathrm{k}}_{\mathrm{S}}\right|=\mathrm{k}$. Therefore, with some manipulation of equations (28), it can be shown that

$$
\begin{aligned}
& \mathrm{K}=2 \mathrm{k} \sin \frac{\theta}{2} \\
& \Delta \mathrm{K}=2 \mathrm{k} \sin \frac{\theta_{\mathrm{c}}}{2} \\
& \mathrm{~K}^{\prime 2}=\mathrm{K}^{2}+(\Delta \mathrm{K})^{2}-2 \mathrm{~K} \Delta \mathrm{K} \cos \delta
\end{aligned}
$$

It can also be shown that $\delta$ is related to $\theta$ and $\theta_{\mathrm{c}}$ as follows:

$$
\cos \delta=-\cos \frac{1}{2}\left(\theta+\theta_{c}\right)
$$

Thus, equation (29c) becomes

$$
\mathrm{K}^{\prime 2}=\mathrm{K}^{2}+(\Delta \mathrm{K})^{2}+2 \mathrm{~K} \Delta \mathrm{K} \cos \left(\frac{\theta+\theta_{\mathrm{c}}}{2}\right)
$$

By adding and subtracting $2 \mathrm{~K} \Delta \mathrm{K}$ to the right side of equation (31) and employing some trigonometric identities, one obtains the following result:

$$
\mathrm{K}^{2}=(\mathrm{K}+\Delta \mathrm{K})^{2}-\left[2 \sin \left(\frac{\theta+\theta_{\mathrm{c}}}{2}\right)\right]^{2} \mathrm{~K} \Delta \mathrm{K}
$$


or, inserting equations (29a) and (29b) into equation (32) and dropping the prime notation:

$$
\mathrm{K}^{2}=4 \mathrm{k}^{2}\left[\left(\sin \frac{\theta}{2}+\sin \frac{\theta_{\mathrm{c}}}{2}\right)^{2}-4 \sin ^{2}\left(\frac{\theta+\theta_{\mathrm{c}}}{4}\right) \sin \frac{\theta}{2} \sin \frac{\theta_{\mathrm{c}}}{2}\right]
$$

or

$$
\mathrm{K}=2 \mathrm{k}\left(\sin \frac{\theta}{2}+\sin \frac{\theta_{\mathrm{c}}}{2}\right)\left[1-\frac{4 \sin ^{2}\left(\frac{\theta+\theta_{\mathrm{c}}}{4}\right) \sin \frac{\theta}{2} \sin \frac{\theta_{\mathrm{c}}}{2}}{\left(\sin \frac{\theta}{2}+\sin \frac{\theta_{\mathrm{c}}}{2}\right)^{2}}\right]^{1 / 2}
$$

Equation (34) describes the scattering process in a medium for which equation (13) is not valid. Measurements of the scattering cross section have agreed in general with theories based on the validity of equation (13), for scattering angles as small as $\approx 10^{\circ}$. These measurements imply that the angle $\theta_{\mathbf{c}}$ is small compared with $10^{\circ}$. It will be shown below that typical values for $\theta_{\mathcal{C}}$ in the lower atmosphere are on the order of $1^{\circ}$ or less. Therefore, equation (34) can be written to a very good approximation as follows:

$$
K=2 k\left(\sin \frac{\theta}{2}+\sin \frac{\theta}{2}\right)
$$

It is clear from equation (35) that even in a nonideal medium, the conventional Bragg condition (eq. (13)) can be used to a good approximation to describe the scattering of sound through angles that are large compared with $\theta_{c}$, but for smaller scattering angles, the conventional Bragg condition must be modified as in equation (35) to include a term which depends only on the properties of the medium and is independent of the acoustic frequency. This simple modification to the Bragg condition can account for the mild frequency dependence which is observed in measurements of the excess attenuation.

To see this, note that when $\mathrm{K}$ is equal to the $\mathrm{K}_{\mathrm{O}}$ of equations (25), by equation (14), $\sin \frac{\theta}{2}=\sin \frac{\theta_{O}}{2}=\frac{\lambda}{2 \mathrm{~L}}=\frac{\pi}{\mathrm{kL}}$, where $\mathrm{L}$ is the outer scale length. Then, from equation (35)

$$
\mathrm{K}_{\mathrm{O}}=2 \mathrm{k}\left(\frac{\pi}{\mathrm{kL}}+\sin \frac{\theta_{\mathrm{c}}}{2}\right)
$$


When the integral in equation (20) is solved by using equations (35) and (36), the result for small $\theta_{\mathrm{c}}$ is

$$
\alpha_{\mathrm{S}}=0.455\left(\frac{\mathrm{C}_{\mathrm{V}}^{2}}{\mathrm{c}^{2}}+0.136 \frac{\mathrm{C}_{\mathrm{T}}^{2}}{\mathrm{~T}^{2}}\right) \mathrm{k}^{1 / 3}\left(\frac{\pi}{\mathrm{kL}}+\sin \frac{\theta_{\mathrm{c}}}{2}\right)^{-5 / 3}
$$

The angle $\theta_{\mathrm{c}}$ is in some sense a measure of the difference between the real atmosphere and the simple model upon which the development in the previous section is based. If the Bragg condition were in fact exactly applicable to the scattering process, then $\theta_{\mathrm{c}}$ would be zero and equation (37) would reduce to equation (35) (or, equivalently, eq. (27)), for which case the excess attenuation coefficient would display the square-law frequency dependence predicted by previous theories. If, however, the $\sin \theta_{c} / 2$ term were on the same order or larger than the $\pi / \mathrm{kL}$ term in equation (37), then the frequency dependence would be much milder than the second power and would approach a cube-root dependence for sufficiently large $\theta_{\mathrm{c}}$. For example, for a plane wave with a frequency of $2 \mathrm{kHz}$ propagating in a medium where $\mathrm{L}$ has a value of $50 \mathrm{~m}$, the $\pi / \mathrm{kL}$ term has a value of $1.7 \times 10^{-3}$. Therefore, if $\theta_{c} \geq 0.2^{\circ}$, the $\sin \theta_{c} / 2$ term will be larger than the $\pi / \mathrm{kL}$ term. That is, under ordinary conditions, if a sound wave is actually scattered in a direction which differs by as little as $0.2^{\circ}$ from the direction it would have been scattered in an idealized medium, then the frequency dependence of the excess attenuation coefficient is much milder than the square law dependence predicted by earlier theories. This is in fact what has been observed experimentally and reported in the papers discussed earlier in the section entitled "Empirical Description of the Excess Attenuation," some of which are discussed further in the next section.

\section{APPLICATION OF THE THEORY AND A COMPARISON}

\section{WITH EXPERIMENTAL RESULTS}

Equation (37) describes the excess attenuation coefficient in terms of the turbulence structure constants, $C_{V}$ and $C_{T}$, the angle $\theta_{c}$, and a scale length $L$. It would be difficult to determine either $\theta_{c}$ or $L$ experimentally and measurements of the structure constants, especially $\mathrm{C}_{\mathrm{V}}$; are difficult to perform. Without such data to support measurements of the excess attenuation, it is difficult to confirm the present calculations conclusively. However, it can be shown that equation (37) does in fact explain those experimental observations reported earlier as well as some other experimental results to be discussed subsequently. 
To date there have only been a small number of propagation experiments conducted over vertical transmission paths in the lower atmosphere and, to the author's knowledge, only one of these had as its objective a systematic investigation of the excess attenuation. This experiment, reported in references 5, 6, and 11, was conducted by Beran, Reynolds, and Gething in Australia in 1969. A sailplane was used as a platform for a sound source which consisted of a speaker mounted flush with the underside of the sailplane wing. The glider was made to spiral down from some initial altitude above a receiver on the ground. Each time the glider passed directly over the receiver, a tone was transmitted from the speaker. The total attenuation was obtained as a function of source height and frequency by measuring the difference in received sound pressure level between successive passes of the glider as it descended. The classical and molecular absorption coefficients were computed from meteorological data recorded by instruments carried on the glider. The difference between the calculated absorption and the total attenuation above spherical spreading was called the excess attenuation.

There were nine successful glider descents in this experiment with data recorded at four frequencies over an altitude range of several hundred meters. The data were reported in a graphical format. Figure 8 is a schematic illustration of a typical plot.

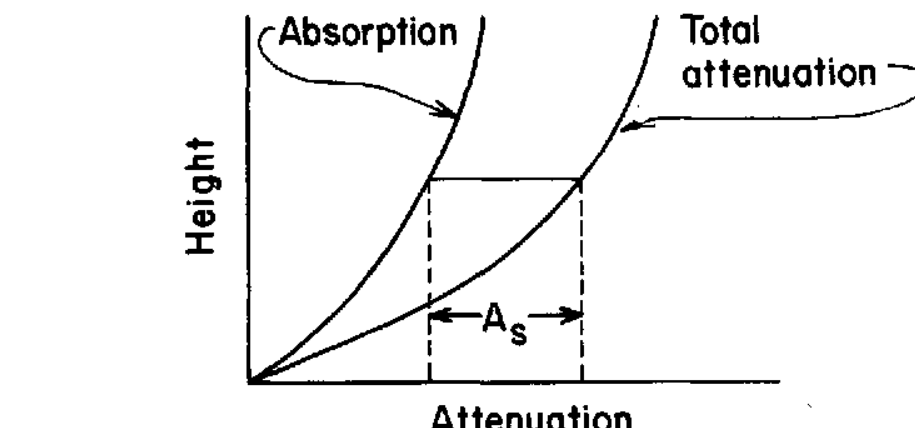

Figure 8. - Schematic representation of data from reference 5.

Both the computed absorption and total attenuation above spherical spreading are plotted as a function of height for a given frequency and glider run. The difference between these two curves represents the excess attenuation. The experimental data from references 5,6 , and 11 have been reanalyzed for five source altitudes from 0.5 to $2.5 \mathrm{~km}$ in multiples of $0.5 \mathrm{~km}$. For each glider descent and for each frequency, the excess attenuation was determined at the five selected source altitudes from the plotted data. (For this purpose, a smooth curve was first fitted by eye through the data points representing the total measured attenuation.) Then the average excess attenuation for a given source altitude and frequency was obtained by averaging the results of all the glider runs at that altitude and frequency. The average accumulated excess attenuation obtained in this way is 
given in table I as a function of frequency for each of the five source altitudes selected for analysis. The uncertainty represents the standard deviation. in averaging over the glider runs.

TABLE I.- AVERAGE ACCUMULATED EXCESS ATTENUATION (dB)

DATA FROM REFERENCE 5

\begin{tabular}{|c|c|c|r|r|}
\hline \multirow{2}{*}{$\begin{array}{c}\text { Height, } \\
\mathrm{m}\end{array}$} & \multicolumn{4}{|c|}{ Average excess attenuation, $\mathrm{dB}$, for frequency of -} \\
\cline { 2 - 5 } & $750 \mathrm{~Hz}$ & $1060 \mathrm{~Hz}$ & $1600 \mathrm{~Hz}$ & \multicolumn{1}{c|}{$2500 \mathrm{~Hz}$} \\
\hline 500 & $1.8 \pm 1.4$ & $2.2 \pm 1.5$ & $3.4 \pm 1.8$ & $4.6 \pm 2.7$ \\
1000 & $3.6 \pm 2.0$ & $4.9 \pm 3.0$ & $8.4 \pm 3.4$ & $9.2 \pm 5.1$ \\
1500 & $5.1 \pm 2.2$ & $5.3 \pm 3.8$ & $10.3 \pm 4.8$ & $12.3 \pm 2.8$ \\
2000 & $5.9 \pm 2.1$ & $6.0 \pm 3.4$ & $10.3 \pm 4.8$ & $16.3 \pm 2.5$ \\
2500 & $6.2 \pm 2.7$ & $6.3 \pm 4.2$ & $12.5 \pm 4.9$ & $15.0 \pm 4.0$ \\
\hline
\end{tabular}

To compare these measured values for the excess attenuation with theory, it is necessary to calculate the accumulated excess attenuation $A_{\mathrm{S}}$ by integrating equation (37) over the propagation path. For a vertical propagation path extending from some source height $\mathrm{h}$ to the ground

$$
A_{S}=\int_{0}^{h} \alpha_{S}(r) d r
$$

where $\alpha_{\mathbf{s}}$ is given by equation (37). All the terms which describe the medium in equation (37) depend to some degree on altitude, but if one assumes the range dependence of the temperature is weak compared with the range dependence of the structure constants $\mathrm{C}_{\mathrm{V}}$ and $\mathrm{C}_{\mathrm{T}}$, then it is only necessary to consider the range dependence of the structure constants in performing the integration in equation (38). Tatarski (ref. 35) gives expressions for the structure constants which display their range dependence explicitly. These formulas, with some notational modifications, are as follows:

$$
\begin{aligned}
& \mathrm{C}_{\mathrm{T}}{ }^{2}=\frac{\mathrm{a}^{2} \kappa^{4 / 3} \mathrm{~T}_{*}^{2}}{\mathrm{r}^{2 / 3}} \\
& \mathrm{C}_{\mathrm{V}}{ }^{2}=\frac{\mathrm{b}^{2} \mathrm{u}_{*}{ }^{2}}{(\kappa \mathrm{r})^{2 / 3}}
\end{aligned}
$$


Here, $\kappa$ is the Von Karman constant with a numerical value of 0.4 and $a$ and $b$ are empirical constants with numerical values of 2.40 and 1.4 , respectively. The quantity $u_{*}$ is called the friction velocity and it depends on the surface roughness. Values of $u_{*}$ are tabulated in standard meteorological references for different kinds of naturally occurring terrains. The quantity $T_{*}$ is a temperature constant which undergoes seasonal variations.

When equations (39) are inserted in equation (37) and the integral in equation (38) is evaluated, the result is as follows. Values for the numerical constants have been combined, along with a neper-to-decibel conversion factor:

$$
\mathrm{A}_{\mathrm{S}}=21.4\left(\frac{\mathrm{u}_{*}^{2}}{\mathrm{c}^{2}}+0.0693 \frac{\mathrm{T}_{*}^{2}}{\mathrm{~T}^{2}}\right)\left(\frac{\pi}{\mathrm{kL}}+\sin \frac{\theta_{\mathrm{c}}}{2}\right)^{-5 / 3} \mathrm{k}^{1 / 3} \mathrm{~h}^{1 / 3}
$$

The glider experiment was conducted over what was described as a vegetated paddock, and from a table in reference 47, a value for the friction velocity $u_{*}$ of $0.4 \mathrm{~m} / \mathrm{sec}$ was selected as representative of this type of terrain. According to Ingard (ref. 17), typical daytime values for the temperature constant $\mathrm{T}_{*}$ range from $0.1^{\circ} \mathrm{C}$ in the winter to $0.5^{\circ} \mathrm{C}$ in the summer. Since this experiment was conducted in the summer (November in Australia), a value of $0.5^{\circ} \mathrm{C}$ was selected for $\mathrm{T}_{*^{*}}$. With these values of $\mathrm{u}_{*}$ and $\mathrm{T}_{*}$, the method of least squares was used to determine the values of $L$ and $\theta_{c}$ for each of the five source heights which gave the best fit of equation (40) to the data in table I. The results of this analysis are presented in table II.

\section{TABLE II.- RESULTS OF LEAST-SQUARES FIT OF}

DATA IN TABLE I TO EQUATION (40)

\begin{tabular}{|c|c|c|}
\hline $\begin{array}{c}\text { Height, } \\
\mathrm{m}\end{array}$ & $\begin{array}{c}\theta_{\mathrm{c}}, \\
\text { deg }\end{array}$ & $\begin{array}{c}\mathrm{L}, \\
\mathrm{m}\end{array}$ \\
\hline 500 & 0.60 & 65 \\
1000 & .40 & 77 \\
1500 & .42 & 100 \\
2000 & .40 & 96 \\
2500 & .41 & 98 \\
\hline
\end{tabular}

In figure 9 , calculated values of $A_{S}$ are plotted against frequency for the five source heights by using values for $\theta_{c}$ and $L$ from table $\Pi$. The discrete points represent data from table $I$. 


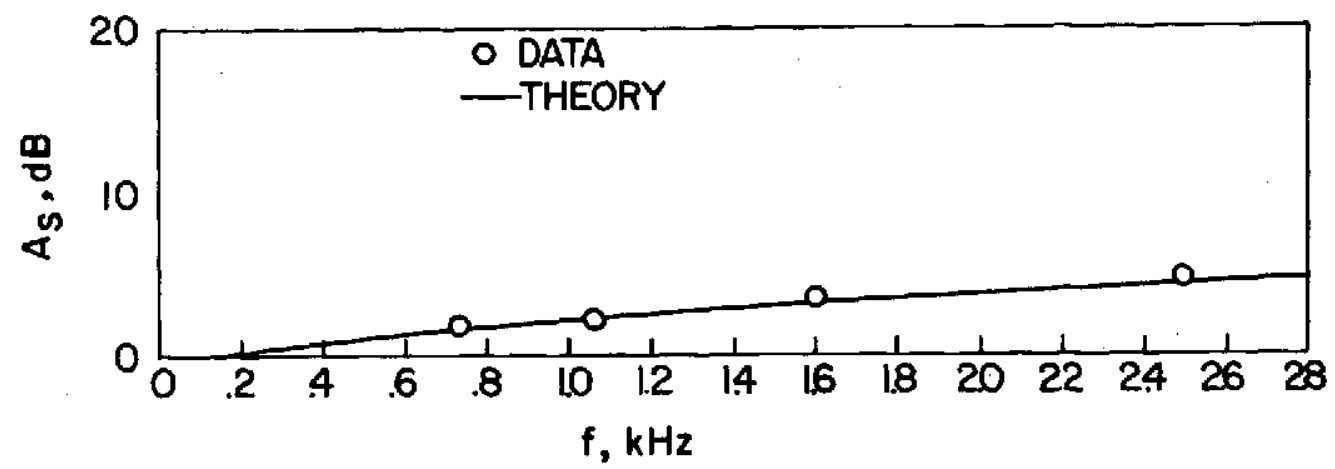

(a) $\mathrm{h}=500 \mathrm{~m} ; \mathrm{I}=65 \mathrm{~m}$; and $\theta_{\mathrm{c}}=0.60^{\circ}$.

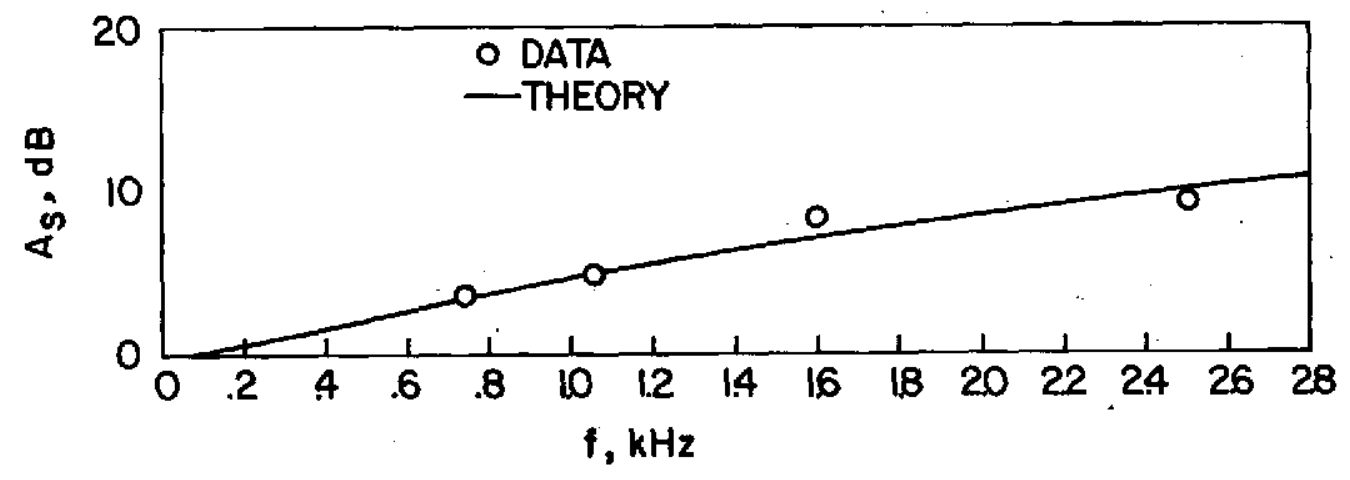

(b) $h=1000 \mathrm{~m} ; \quad I=77 \mathrm{~m}$; and $\theta_{c}=0.40^{\circ}$.

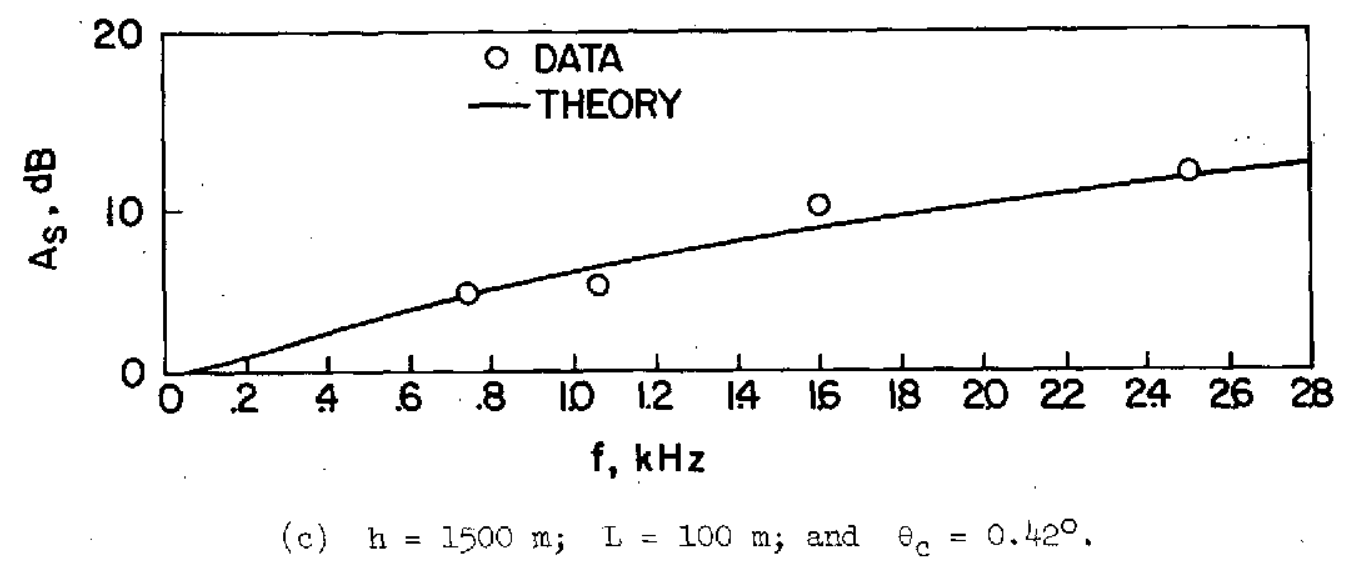

Figure 9.- variation of $A_{S}$ with $f^{\prime}$ (data from ref. 5). 


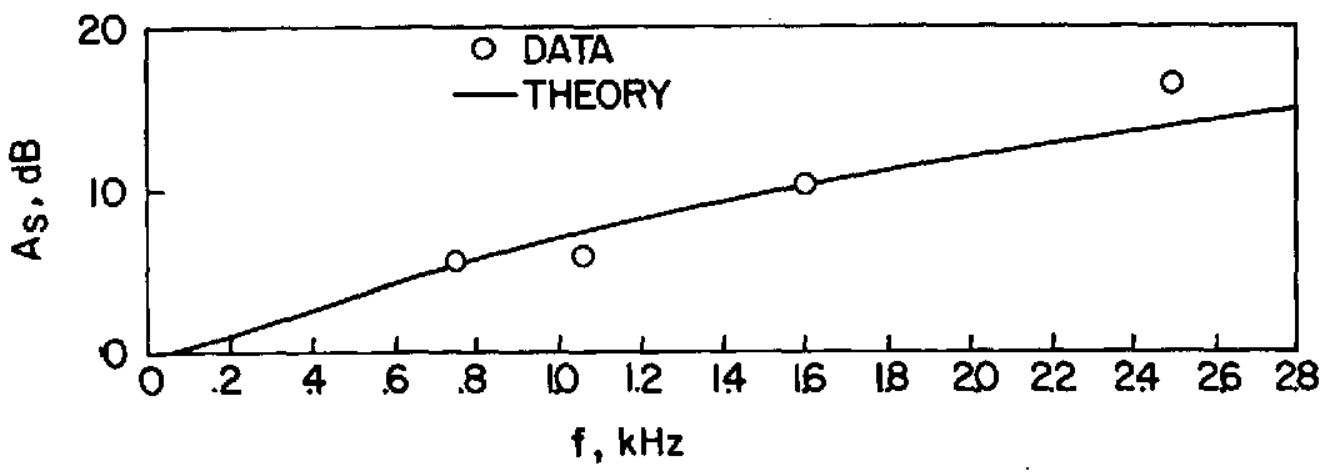

(d) $\mathrm{h}=2000 \mathrm{~m} ; \mathrm{L}=96 \mathrm{~m}$; and $\theta_{\mathrm{c}}=0.40^{\circ}$.

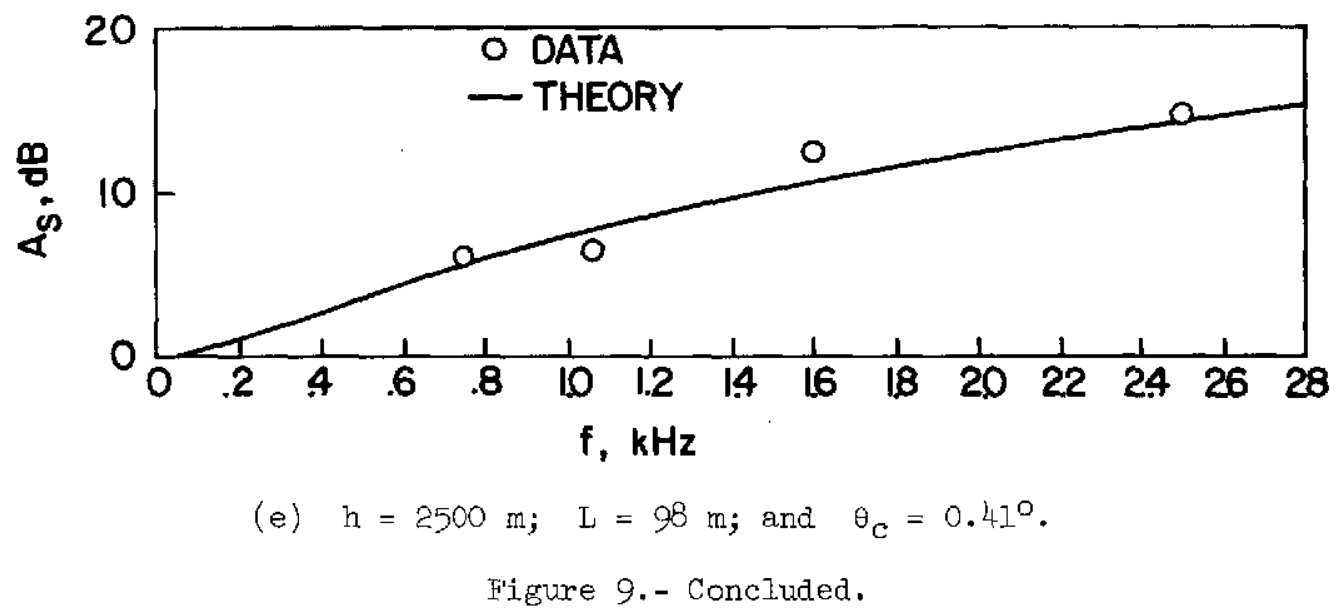

The large standard deviation in this experiment is typical of outdoor sound propagation measurements and reflects the fact that the turbulence intensity at a given altitude fluctuated a great deal between successive glider descents. The standard deviation illustrates the dynamic nature of the atmosphere which makes it difficult to conduct a systematic empirical investigation of the excess attenuation. Although the data are fairly coarse, figure 9 indicates that the results of this experiment agree with the present theory within experimental error. The outer scale lengths which give the best agreement between theory and experiment are quite representative of the outer scale lengths which generally characterize turbulence in the atmosphere and the $\theta_{c}$ values of best fit are small, as expected. Also, the frequency dependence of the measured excess attenuation lies between the second power and the one-third power, as predicted by equation (37).

The structure constants, $C_{V}$ and $C_{T}$, were not measured in this experiment; however, there were measurements made of the wind shear, which is related to $\mathrm{C}_{\mathrm{V}}$. Figure 10 , taken from reference 5 , is a plot of excess attenuation against wind shear squared for three different frequencies. (In the original paper, data taken at $2500 \mathrm{~Hz}$ were also 


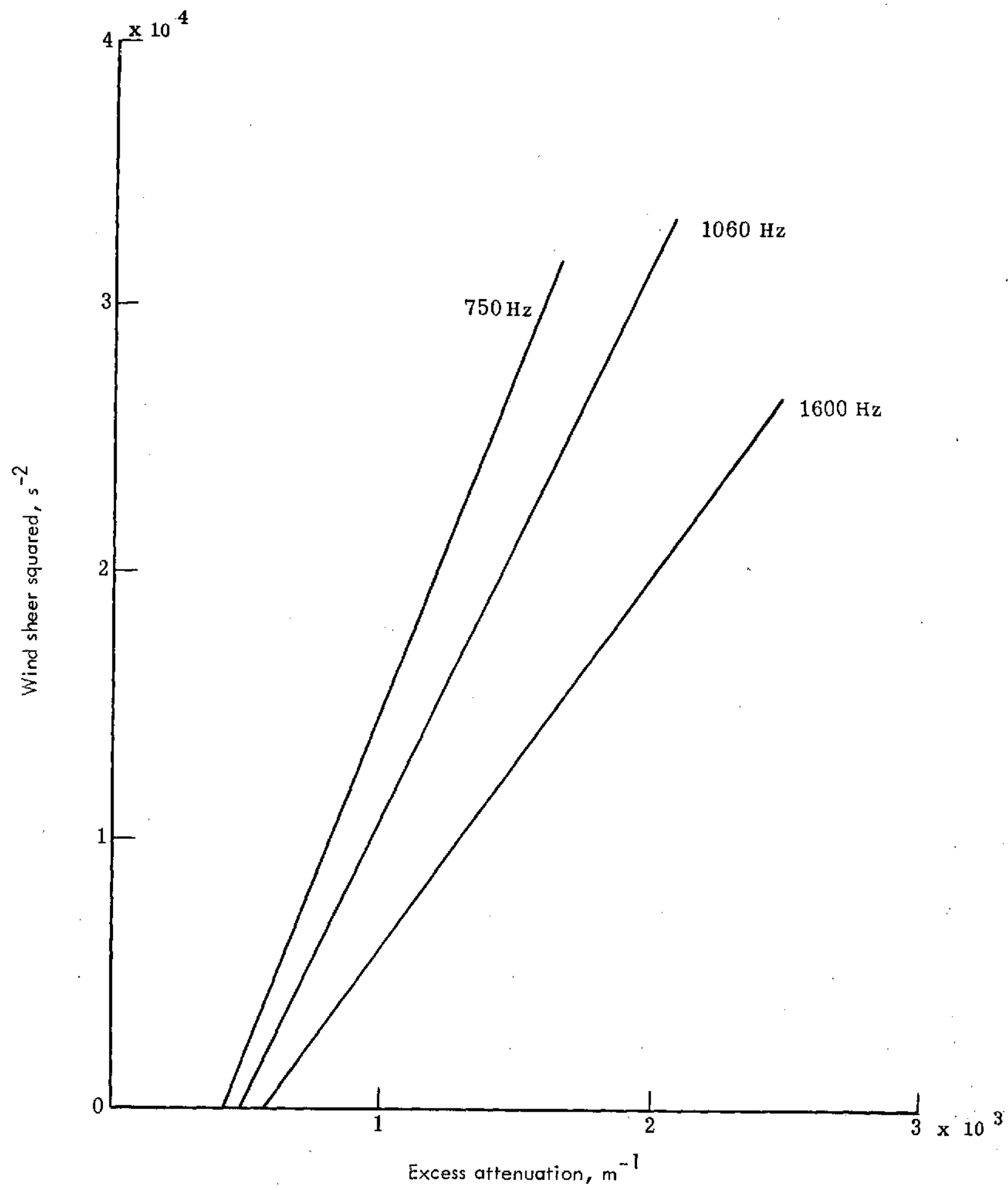

Figure 10.- Regression curves for variation of average excess attenuation with average wind shear squared. (From ref. 5.) 
plotted but the quality of the $2500-\mathrm{Hz}$ data was questioned.) These plots indicate that as the square of the wind shear increases, the excess attenuation increases, but the fact that the regression curves do not intersect the origin led Beran and his collaborators to conclude that other effects might play a dominant role under conditions of low wind shear. This result is also predicted by equation (37). Because $\mathrm{C}_{\mathrm{V}} / \mathrm{c}^{2}$ is generally of the same order of magnitude as $\mathrm{C}_{\mathrm{T}} 2 / \mathrm{T}^{2}$ in the atmosphere, the numerical factor of 0.136 in front of the temperature term in equation (37) causes the wind shear term to dominate most of the time. Therefore, the excess attenuation usually depends in a relatively sensitive way on the wind shear. However, the temperature term keeps the excess attenuation from going to zero as the wind shear goes to zero, and under conditions of very low shear, when $\frac{\mathrm{C}_{\mathrm{V}}^{2}}{\mathrm{c}^{2}} \ll 0.136 \frac{\mathrm{C}_{\mathrm{T}}{ }^{2}}{\mathrm{~T}^{2}}$, the excess attenuation will not depend as strongly on the wind shear. In fact, in Beran's experiment the fairly strong correlation between wind shear squared and the excess attenuation was observed in all glider descents except one. For that run, the wind shear was described as low to moderate and a fairly strong convective activity was reported. Under such conditions it is to be expected that the $\mathrm{C}_{\mathrm{T}}$ term would dominate the $\mathrm{C}_{\mathrm{V}}$ term in equation (37) and the excess attenuation would not depend as strongly on wind shear.

The atmosphere is a very unsatisfactory medium in which to conduct a sound propagation experiment because meteorological fields and turbulence conditions can undergo severe fluctuations in relatively short periods of time in a way which cannot be controlled by the experimenter. It would be much more desirable to conduct a propagation experiment in an environment featuring turbulence conditions and meteorological fields which the experimenter could alter independently. Such a controlled environment, while unattainable out of doors, can be generated in the laboratory. The technique is to inject a grid of parallel, cylindrical bars into a wind tunnel in which a laminar flow has been established, and cause turbulence to be generated downstream from the grid. The scale of turbulence at any point downstream from the grid can be altered by changing the grid geometry (see ref. 48) and the turbulence intensity can be varied by changing the dynamic pressure in the tunnel.

The scattering theory is not restricted to atmospheric turbulence but applies to any turbulent medium with a low mean flow speed in which the acoustic wavelengths are small compared with the outer scale of turbulence, provided the Reynolds number is sufficiently large for the inertial subrange to occupy a fairly large part of the turbulence spectrum. By using ultrasonic frequencies in a wind tunnel so that the ratio of the turbulence scale to the acoustic wavelength is about the same as in an atmospheric experiment in which audio frequencies are used, it is possible to perform a "scale model" scattering experiment under controlled laboratory conditions. Such an experiment has been performed by Schmidt and is reported in reference 49 . 
The experimental arrangement in Schmidt's experiment is illustrated in figure 11. An ultrasonic transmitter and receiver were mounted in opposite walls of a wind-tunnel test section $30 \mathrm{~cm}$ apart so that an acoustic signal traveling from the transmitter to the receiver would travel across the flow. Each data point was taken in a two-step procedure. First, an acoustic signal was transmitted through laminar flow to the receiver to establish a reference sound pressure level. Then a grid of cylindrical bars was placed in the flow upstream from the transmission path and the same signal was passed through the turbulence induced by the grid. The signal passing through the turbulent medium suffered an attenuation relative to the signal passing through the laminar flow. This excess attenuation was due to the effects of the turbulence. (The temperature and humidity were unaffected by the presence of the grid so that the molecular and classical absorption were the same for both the laminar and turbulent cases.)

Schmidt's experiment was conducted to test the theory developed by Müller and Matschat (ref. 27). This theory, which was discussed earlier, culminated in an expression which reduced to equation (7a) for the case of sound propagating perpendicular to the eddy axes with wavelengths small compared with the turbulence scale. For convenience, equation (7a) is repeated here

$$
\alpha_{S}=20\left(1-\frac{2}{\pi}\right)(\log e) \frac{\overline{\Delta v^{2}}}{c^{2}} L k^{2}
$$

Like the theories of Lighthill and Batchelor which are represented by equation (8), the theory of Müller and Matschat predicts that the coefficient of attenuation due to inertial scattering depends on the square of the acoustic frequency and on the mean-square turbulence fluctuation velocity in the direction of sound propagation $\overline{\Delta v^{2}}$. To test this dependence, a series of measurements were made by Schmidt in which the acoustic frequency and $\mathrm{q}$, the dynamic pressure of the wind tunnel, were varied. The dynamic pressure is proportional to the square of the mean flow velocity $\bar{v}$, and $\bar{v}$ is proportional to the mean turbulence fluctuation velocity, $\widehat{\Delta v}$. Therefore, the mean-square fluctuation velocity is proportional to the dynamic pressure and, according to the earlier theories, the

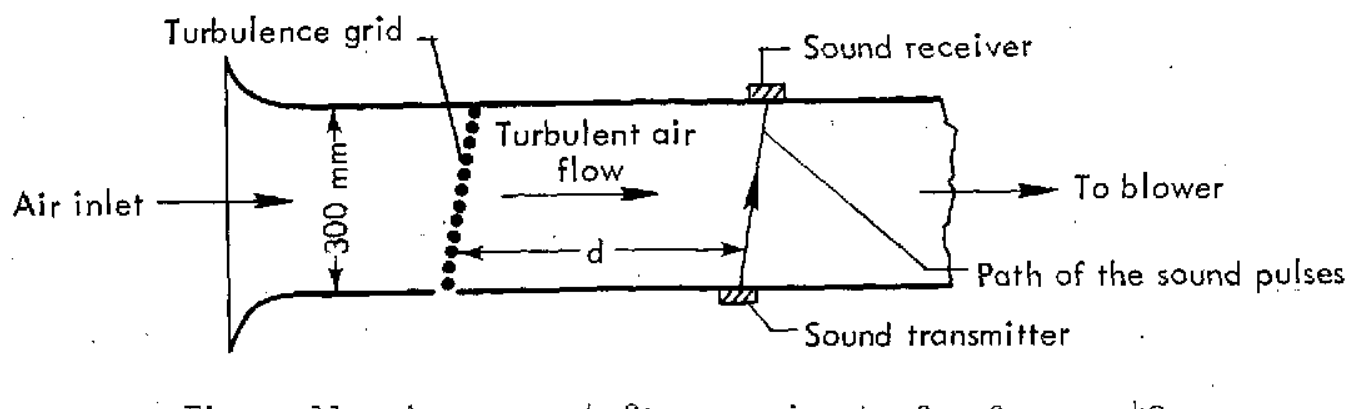

Figure 11.- Arrangement for experiment of reference 49. 
excess attenuation should be directly proportional to $\mathrm{qf}^{2}$, where $f$ is the acoustic frequency.

The results of one series of measurements are presented in figure 12(a), which is taken from reference 49 . The grid cylinders were $0.15 \mathrm{~cm}$ in diameter and separated by a distance of $2.50 \mathrm{~cm}$ in the me measurements. The transmission path was $30.0 \mathrm{~cm}$ long and located $5.0 \mathrm{~cm}$ downstream from the grid. For these turbulence conditions, the excess attenuation increased linearly with $\mathrm{qf}^{2}$ as predicted by the earlier theories; however, the measurements began to deviate from this linear dependence at higher values of $\mathrm{qf}^{2}$ when they were repeated under different conditions of turbulence. See, for example, figures $12(\mathrm{~b})$ to $12(\mathrm{~d})$.

The earlier theories seemed to break down systematically, according to the following scheme. The greater the ratio of the grid cylinder diameter to the distance between the transmission path and the grid, the greater the deviation between experiment and theory. This ratio is denoted by $R$ in figure 12 . The turbulence intensity is greater near the grid than further downstream. (At a great distance downstream the turbulence would all be dissipated and the flow would again be laminar.) The turbulence intensity also increases with increasing grid cylinder diameter. (In the limiting case of zero cylinder diameter, the flow would be laminar.) Therefore, the experimental results exhibit the greatest deviation from the linear $\mathrm{qf}^{2}$ dependence when the turbulence intensity is the greatest.

From the data presented in reference 49 , it is not possible to tell whether the linear $\mathrm{f}^{2}$ dependence is breaking down at high $\mathrm{qf}^{2}$ values in figures $12(\mathrm{~b})$ to 12 (d) or whether it is the linear $q$ (that is, $\overline{\Delta v^{2}}$ ) dependence which is breaking down. To separate the effects of varying $q$ from the effects of varying $f$, the data from figures 12 (b) to 12 (d) were analyzed in the present study as follows: First, the dynamic pressure, frequency, and measured excess attenuation were tabulated for each data point. (The excess attenuation was read directly from the ordinate axis of the graphs while the dynamic pressure was computed by dividing the $\mathrm{qf}^{2}$ abscissa coordinate of each data point by $\mathrm{f}^{2}$, which is given for each point.) In figure 13 the excess attenuation is plotted against frequency for three different values of $\mathrm{q}$. In figure 14 , the excess attenuation is plotted against dynamic pressure for various frequencies.

The data are much more illuminating when presented in this way. First, note that in figure 13, the excess attenuation does depart from an $\mathrm{f}^{2}$ dependence and approaches a weaker frequency dependence at relatively high frequencies. This high frequency departure from the $\mathrm{f}^{2}$ dependence increases with increasing dynamic pressure. The excess attenuation also departs from a linear $q$ dependence at higher $q$ values, as can be seen in figure 14, and approaches a milder dependence on $q$ than $q$ to the first power. The higher the frequency, the more pronounced this departure is. 


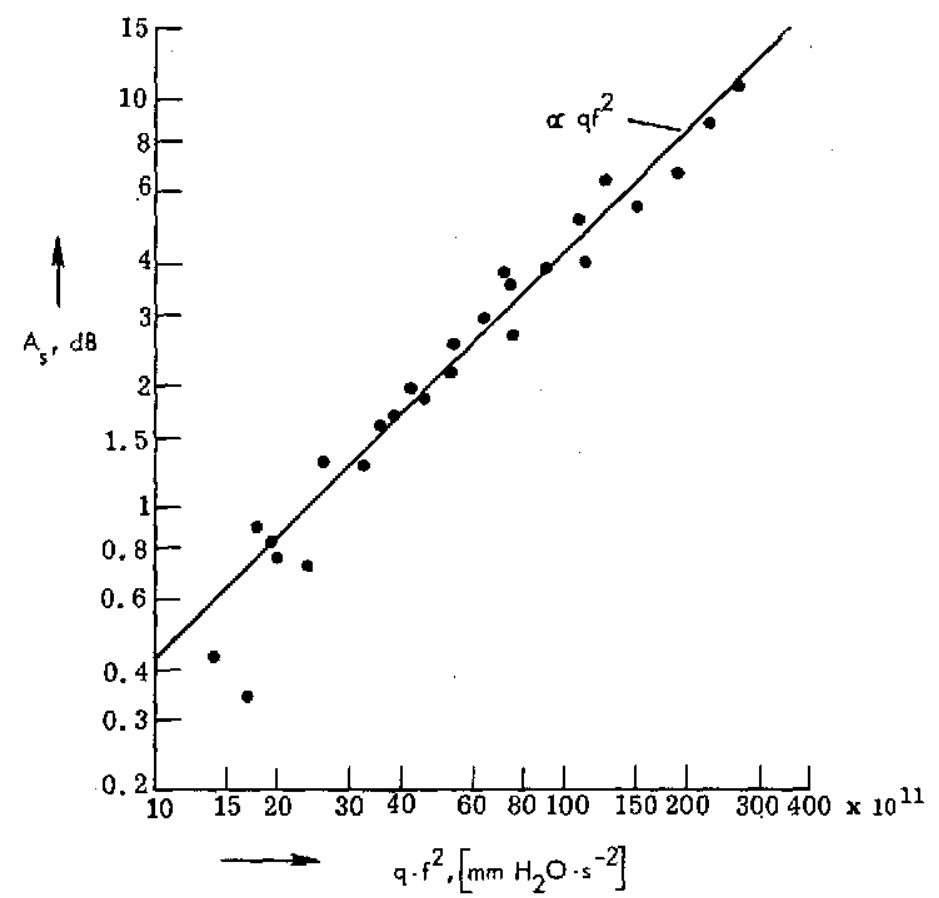

(a) $\mathrm{R}=0.03 ; \mathrm{D}=0.15 \mathrm{~cm} ; \mathrm{d}=5.0 \mathrm{~cm}$; and $\mathrm{M}=2.50 \mathrm{~cm}$.

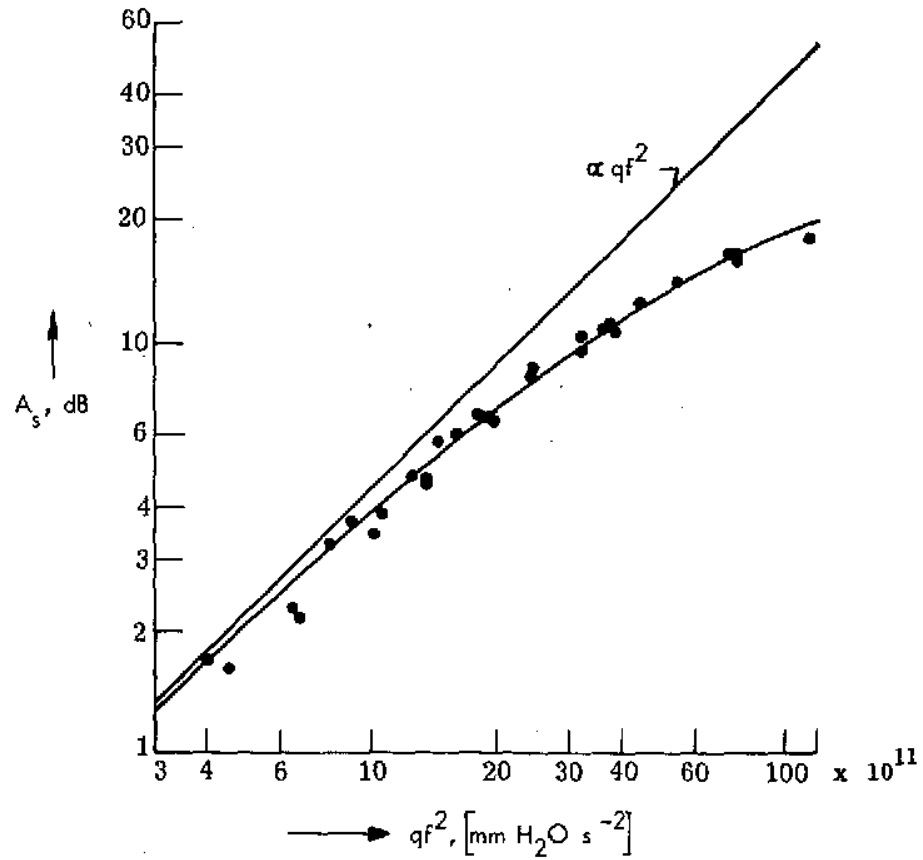

(b) $R=0.05 ; D=1.00 \mathrm{~cm} ; d=20.0 \mathrm{~cm}$; and $M=2.50 \mathrm{~cm}$.

Figure 12.- Data from Schmidt (rer. 49). 


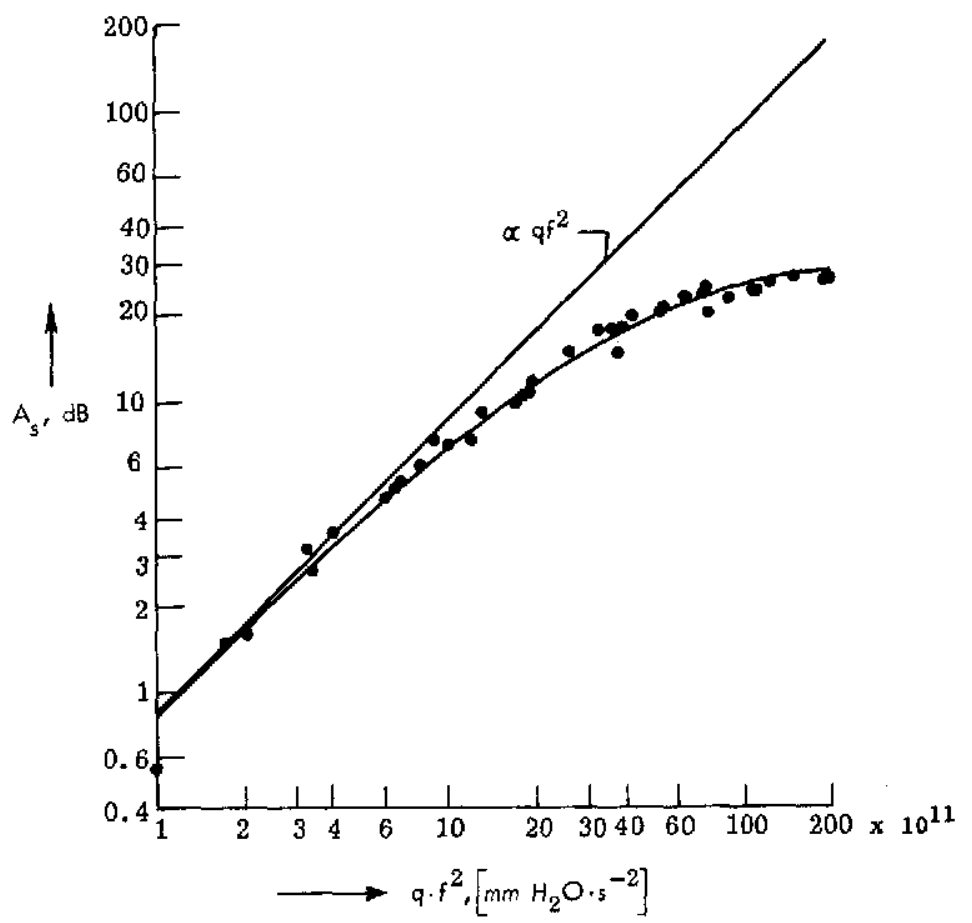

(c) $R=0.08 ; D=0.40 \mathrm{~cm} ; d=5.0 \mathrm{~cm}$; and $M=2.50 \mathrm{~cm}$.

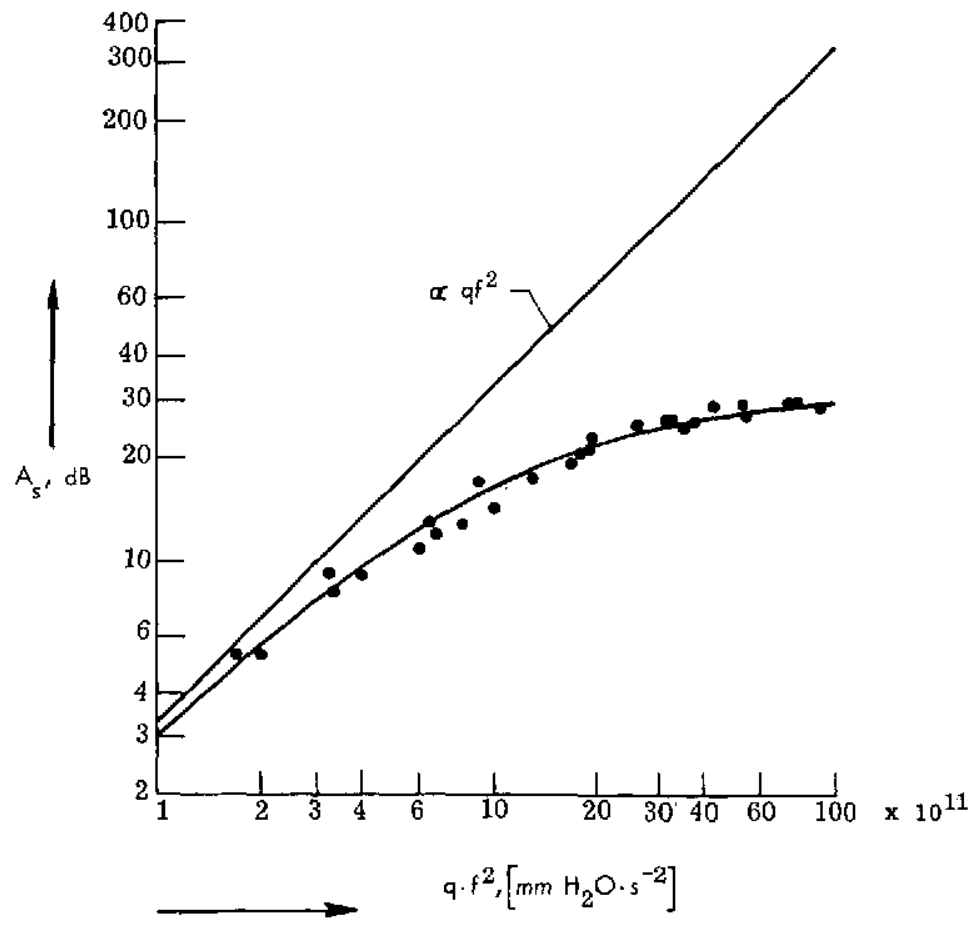

(d) $\mathrm{R}=0.20 ; \mathrm{D}=1.00 \mathrm{~cm} ; \mathrm{d}=5.0 \mathrm{~cm}$; and $\mathrm{M}=2.50 \mathrm{~cm}$.

Figure 12.- Concluded. 


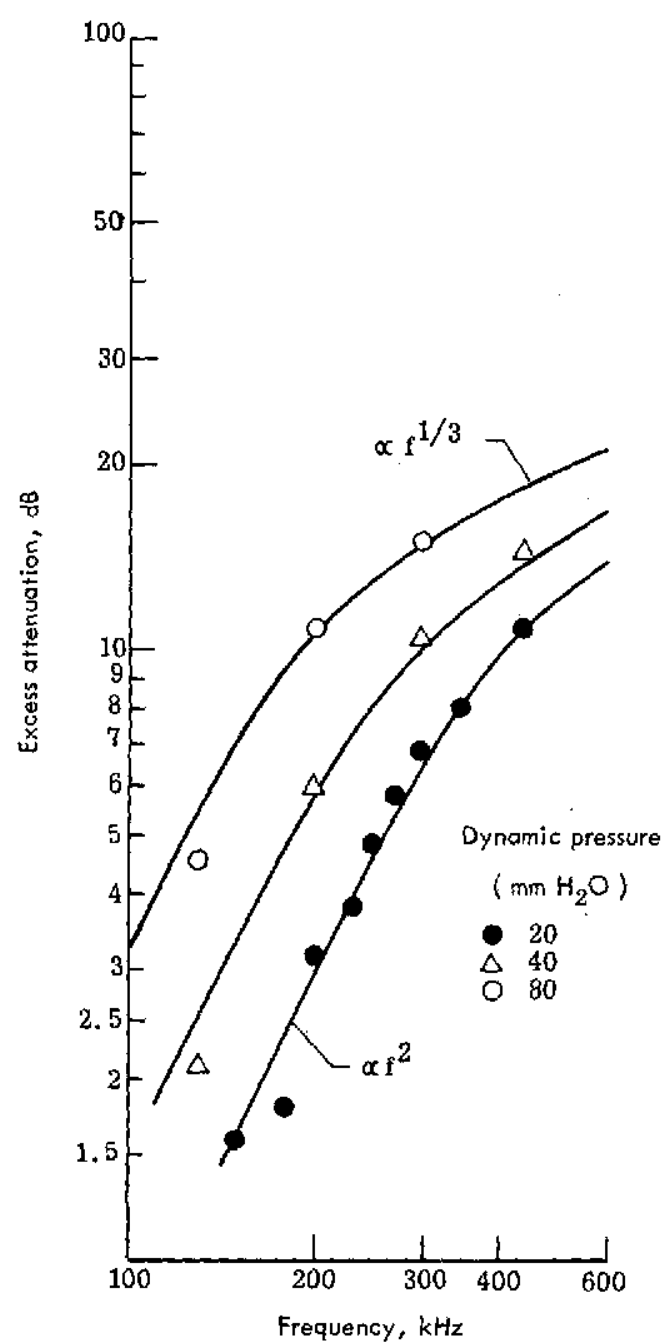

(a) $\begin{aligned} & D=1.00 \mathrm{~cm} ; M=2.50 \mathrm{~cm} ; \\ & Q=20 \mathrm{~cm} ; \text { and } R=0.05 .\end{aligned}$

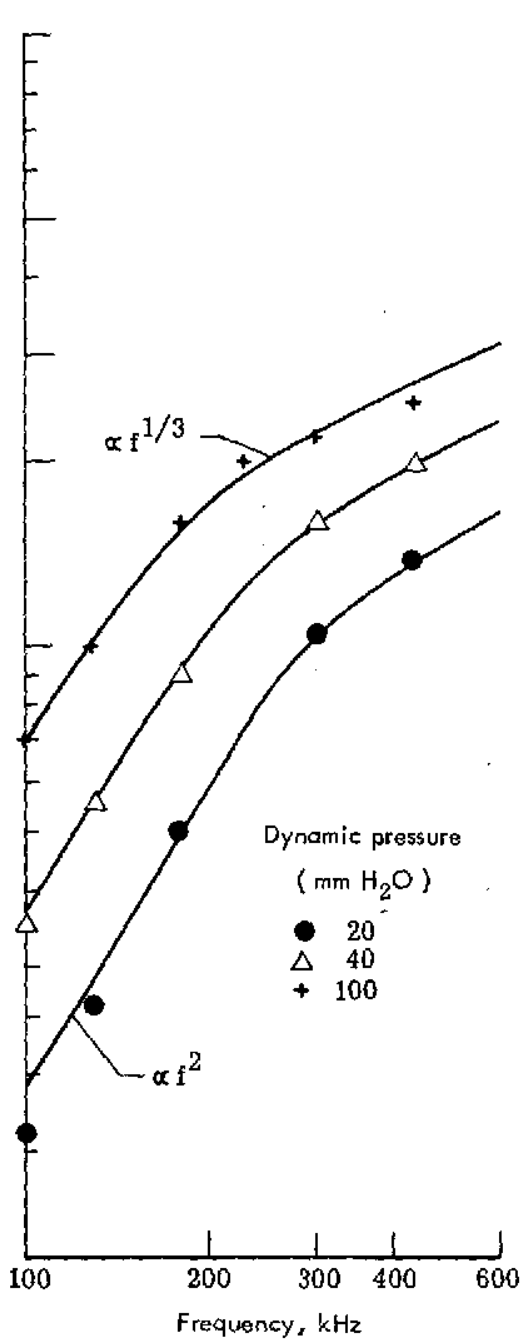

(b) $D=0.40 \mathrm{~cm} ; M=2.50 \mathrm{~cm}$; $d=5.0 \mathrm{~cm}$; and $R=0.08$.

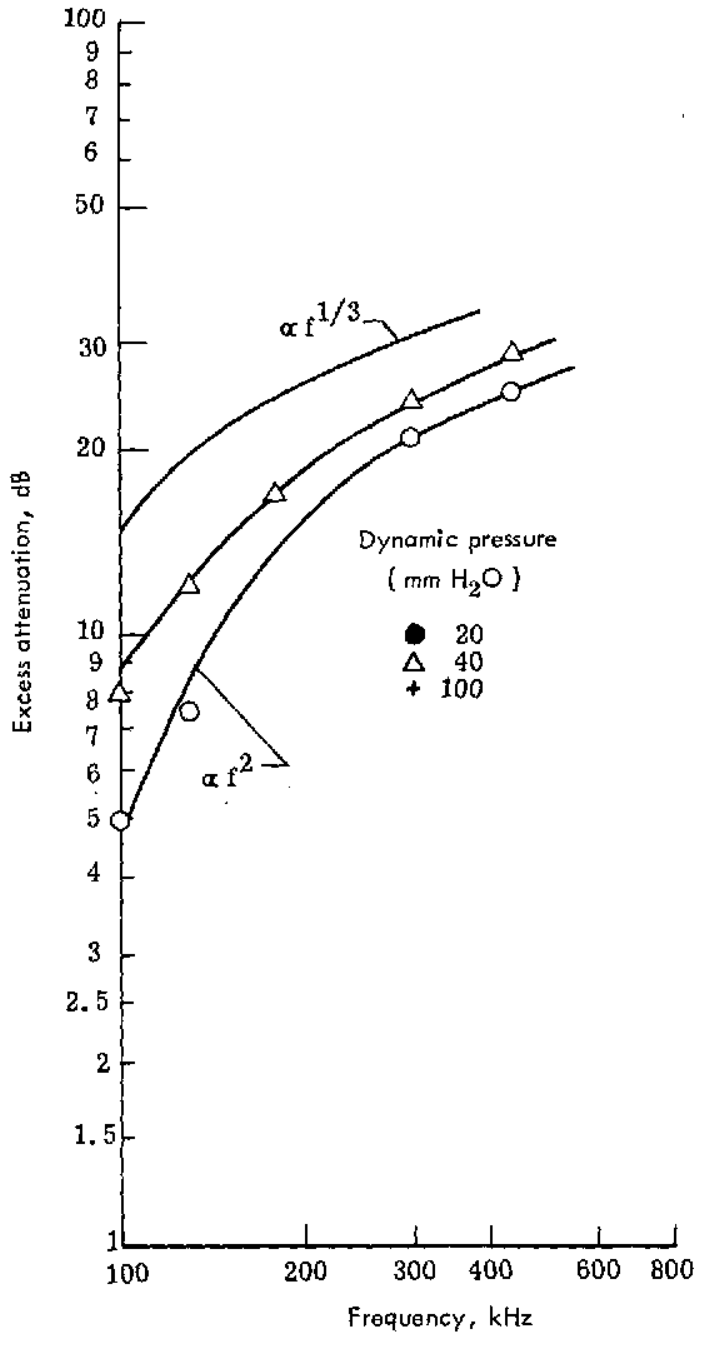

(c) $D=1.00 \mathrm{~cm} ; \quad M=2.50 \mathrm{~cm}$; $d=5.0 \mathrm{~cm}$; and $R=0.20$.

Figure 13.- Variation of excess attenuation with frequency data from figure 12. 


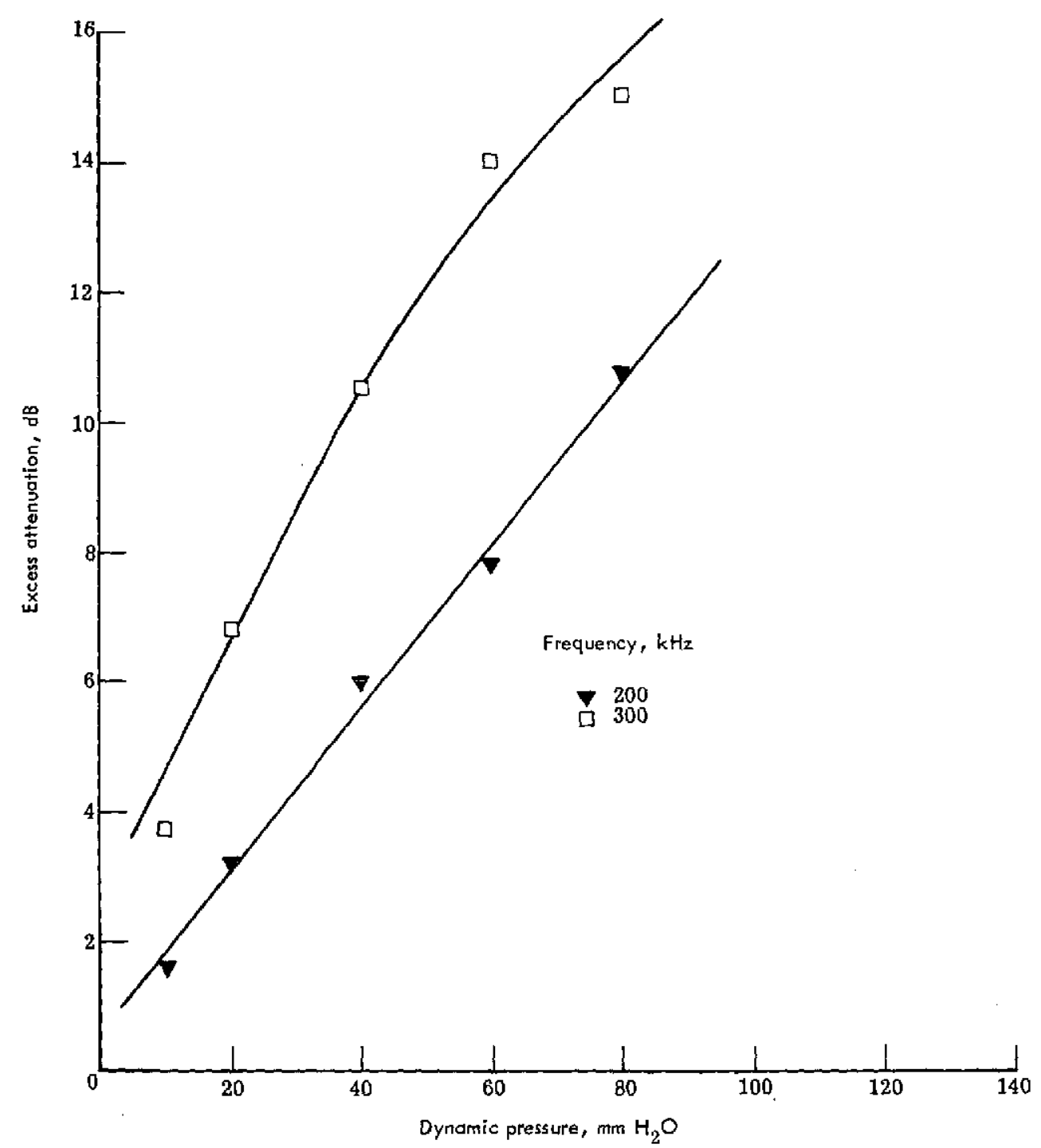

Figure 14.- Variation of excess attenuation with dynamic pressure data from figure 12. 


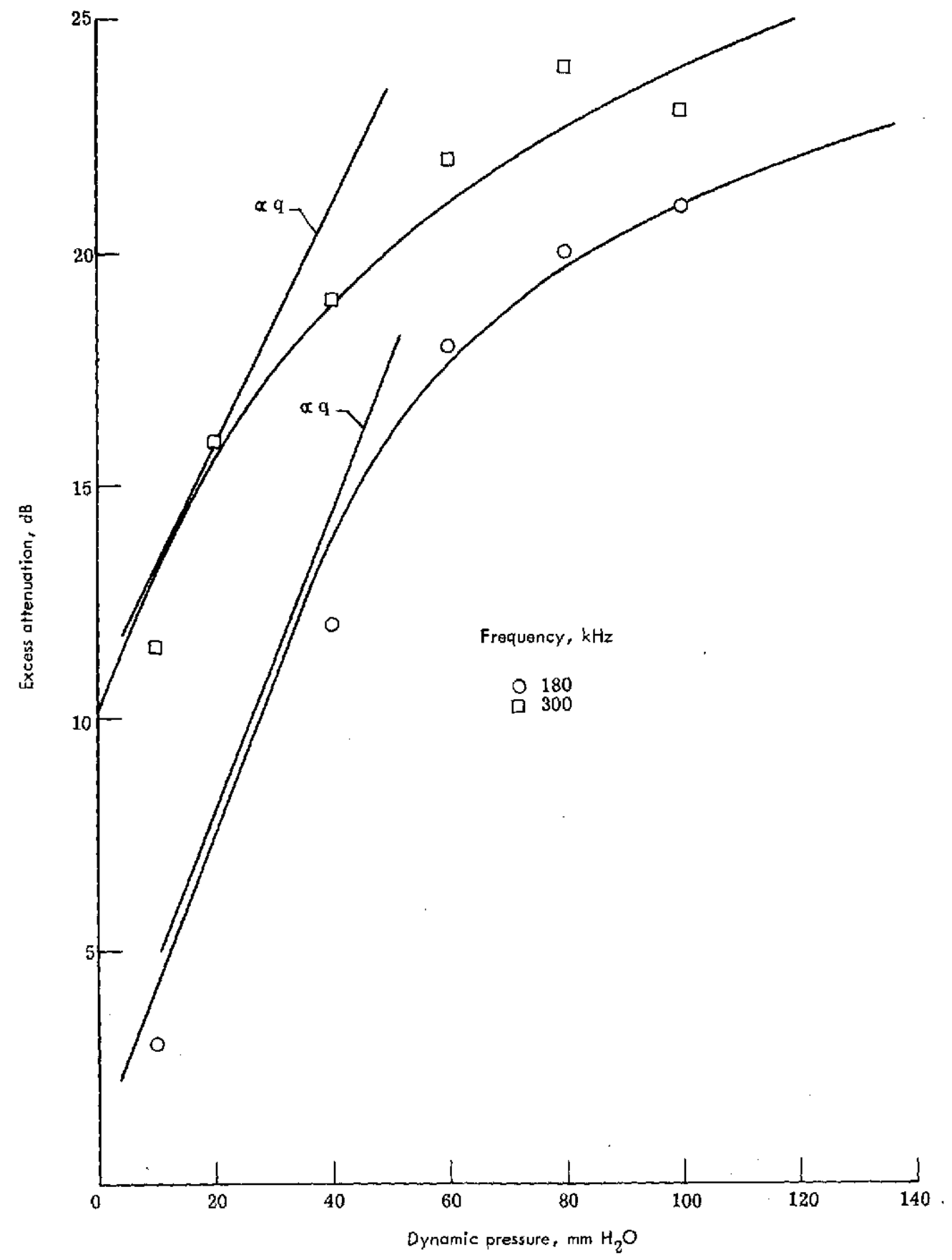

Figure 14.- Contimed. 


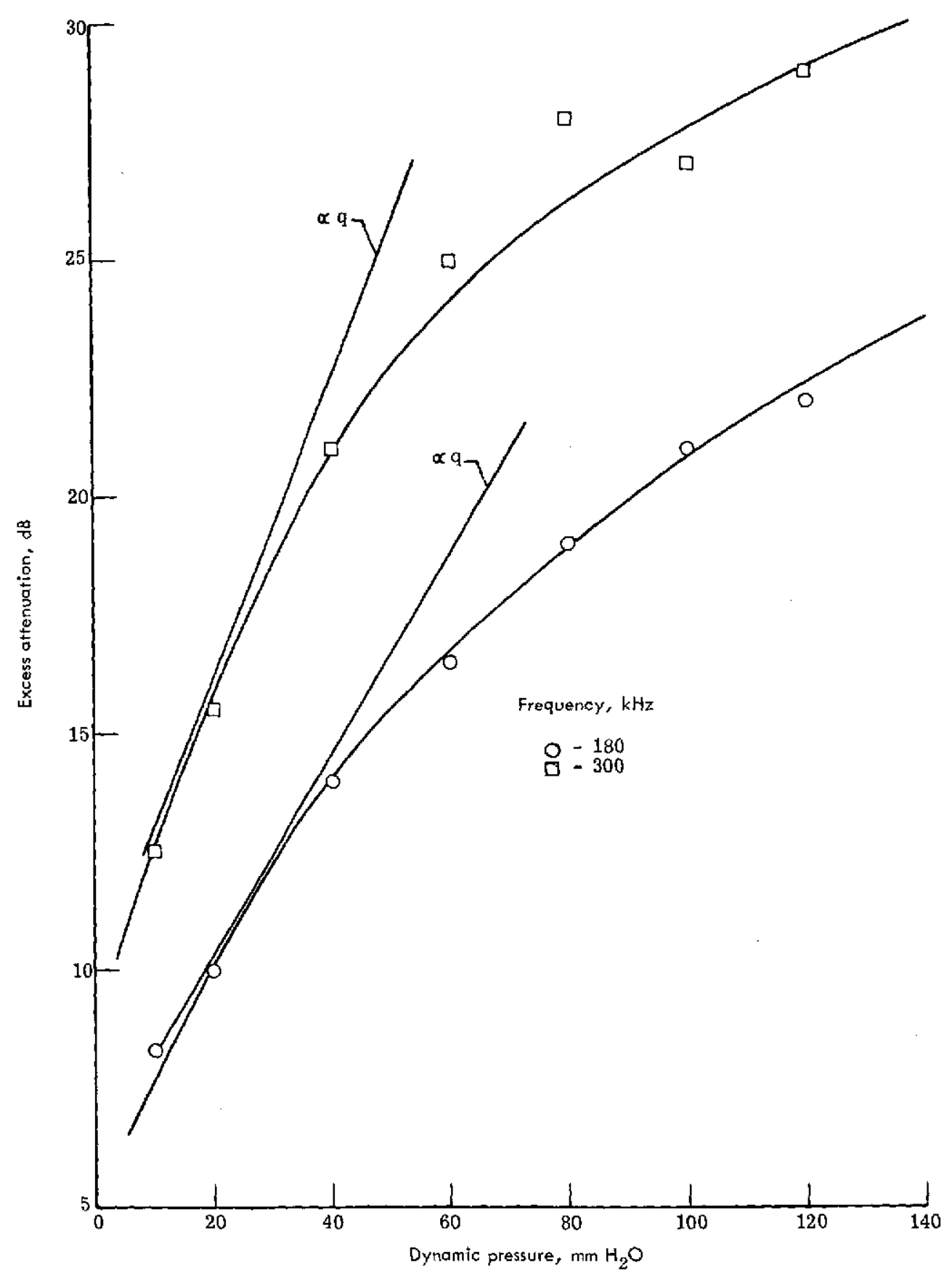

Figure 14.- Concluded. 
The excess attenuation theory developed in this report can explain the $f$-dependence and the $\mathrm{q}$-dependence which is displayed in figures 13 and 14. First, note that in Schmidt's experiment, there were no temperature-induced inhomogeneities in the wind-tunnel flow so the $\mathrm{C}_{\mathbf{T}}$ term in equation (37) can be neglected

$$
\alpha_{\mathrm{S}}=0.455\left(\frac{\mathrm{C}_{\mathrm{V}}^{2}}{\mathrm{c}^{2}}\right)_{\mathrm{k}}^{1 / 3}\left(\frac{\pi}{\mathrm{kL}}+\sin \frac{\theta_{\mathrm{c}}}{2}\right)^{-5 / 3}
$$

Consider the frequency dependence which equation (41) predicts. If the frequency is sufficiently low for the $\pi / \mathrm{kL}$ term to dominate the $\sin \theta_{\mathrm{c}} / 2$ term, then $\alpha_{\mathrm{S}}$ will approach a square-law frequency dependence. As the frequency is increased, the $\pi / \mathrm{kL}$ term decreases and $\alpha_{\mathrm{S}}$ exhibits a weaker frequency dependence and approaches $\mathrm{f} 1 / 3$ for very high frequencies. This is in fact what was observed experimentally, as may be seen in figure 13; the higher the frequency, the milder the frequency dependence.

This trend is accentuated when the turbulence intensity is increased, either by increasing the dynamic pressure $q$ or by increasing the grid cylinder diameter $D$ or by decreasing the distance $d$ between the turbulence grid and the transmission path. When the dynamic pressure is increased or the propagation path is moved closer to the grid, the sound beam encounters a flow field which is more irregular than it would encounter under milder turbulence conditions. The $\theta_{c}$ term in equation (41) is therefore increased. According to reference 49, the turbulence scale is related to the grid cylinder diameter and the separation between the grid and the transmission path as follows:

$$
L=0.125 \mathrm{D} \sqrt{1+0.92 \frac{\mathrm{d}}{\mathrm{D}}}
$$

Increasing the grid cylinder diameter $D$ therefore increases the turbulence scale. Thus, the net effect of increasing $D$ or $q$ or decreasing $d$ is to increase the $\sin \theta_{c} / 2$ term in equation (41) relative to the $\pi / \mathrm{kL}$ term and thereby decrease the strength of the frequency dependence. This is the result observed in Schmidt's experiment.

Now, consider how the excess attenuation depends on the dynamic pressure $q$. The dynamic pressure is proportional to $\overline{\Delta v^{2}}$ as was stated above. For small $q$, the turbulence field exhibits a very small departure from laminar flow and the $\theta_{c}$ term in equation (41) is small compared with the $\pi / \mathrm{kL}$ term. As $q$ increases, $\overline{\Delta v^{2}}$ increases proportionately and therefore so does $\mathrm{C}_{\mathrm{V}}{ }^{2}$, by equation (26a). Thus, for small $\mathrm{q}, \alpha_{\mathrm{S}}$ increases approximately linearly with $\mathrm{C}_{\mathrm{V}}{ }^{2}$ (that is, with $\mathrm{q}$ ) by equation (41). For larger $q$, the flow becomes more irregular and the $\sin \theta_{c} / 2$ term in equation (41) begins to have an effect. As $q$ is increased, both $C_{V}{ }^{2}$ and $\theta_{c}$ increase but although 
increasing $\mathrm{C}_{\mathrm{V}}{ }^{2}$ causes $\alpha_{\mathrm{s}}$ to increase, increasing $\theta_{\mathrm{c}}$ causes $\alpha_{\mathrm{s}}$ to decrease, with the net result that at high $q$ values, the rate of increase of $\alpha_{\mathrm{s}}$ with $q$ slows down. This saturation effect is the effect that was observed experimentally in Schmidt's measurements, as can be seen in figure 14 .

It may be mentioned here that Beran et al. (ref. 5) reported that the excess attenuation increased as a nonlinear function of wind shear squared in his experiment and in comparing the results of field measurements with the theory of Lighthill (ref. 40), Ingard and Wiener (ref. 43) reported a much milder dependence on wind effects than would be expected on the basis of the Lighthill theory.

One of the objectives of the NASA research into the atmospheric excess attenuation problem is to gain some detailed knowledge of the effect of turbulence on sound radiated from an aircraft. An outdoor propagation experiment is reported in references 7 to 10 in which an aircraft was in fact used as the sound source. In this experiment, a propellerdriven aircraft made a combination of level flybys and dive passes over a microphone station on the ground. The experiment was conducted at two field sites, one at LaFox, Illinois, about 40 miles west of Chicago, and one at a leased airfield about 25 miles west of Phoenix, Arizona. Measurements at the Illinois site were made during the period of July 1956 to October 1956, and measurements at the Arizona site were made during the period of November 1956 to February 1957. The Arizona site was described as flat, with sparse vegetation whereas the Illinois site was described as typical farm country with corn growing along one side of the landing strip and the stubble from a harvested stand of winter wheat on the other. The strip was overgrown with coarse grass 6 to 10 inches high. Thus, measurements were made over a fairly wide range of meteorological and ter rain conditions. The 110 flights yielded a total of 40000 data points for the flyby data and 130000 data points for the dive-pass data.

An excess attenuation was consistently observed in this experiment and when average values of the excess attenuation coefficient measured at both field sites were plotted as a function of frequency to the one-third power, a linear relationship was observed. (See fig. 3.) This linear dependence on $\mathrm{f}^{1 / 3}$ suggests that in this experiment the average outer length $\mathrm{L}$ or the average value of the angle $\theta_{\mathrm{c}}$, or both, were sufficiently large for the $\sin \theta_{\mathrm{c}} / 2$ term in equation (37) to dominate the $\pi / \mathrm{kL}$ term to the extent that the $\pi / \mathrm{kL}$ term could be effectively neglected. The slope of the curve in figure 3 is 0.11 and thus equation (6a) is given in references 7 to 10 as an empirical formula for the average excess attenuation coefficient ( $f$ in $\mathrm{Hz}$ ):

$$
\overline{\alpha_{s}}=0.11 \mathrm{f}^{1 / 3} \mathrm{~dB} / 1000 \mathrm{ft}
$$


To compare this empirical result with the present theory, it is necessary to calculate the average excess attenuation suffered by a sound wave propagating from a height $h$ to a height $h_{0}$, as follows:

$$
\overline{\alpha_{s}}=\frac{1}{\mathrm{~h}-\mathrm{h}_{\mathrm{o}}} \int_{\mathrm{h}_{\mathrm{o}}}^{\mathrm{h}} \alpha_{\mathrm{s}} \mathrm{dr}
$$

where $\alpha_{\mathrm{s}}$ can be expressed in terms of $\mathrm{u}_{*}$ and $\mathbf{T}_{*}$ as was done above. When this integral is evaluated, the result is

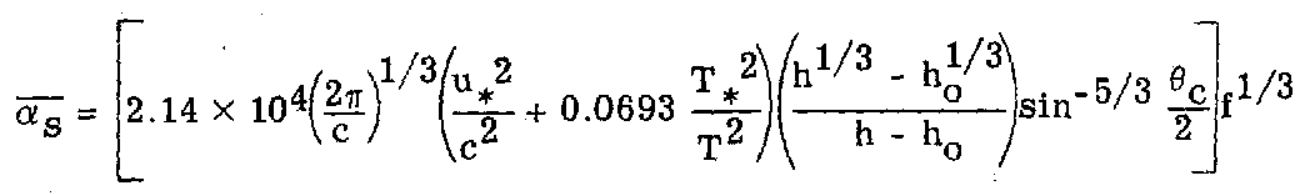

where, when all terms are expressed in English units, $\widetilde{\alpha}_{\mathrm{s}}$ is in units of $\mathrm{dB} / 1000 \mathrm{ft}$. The $\pi / \mathrm{kL}$ term from equation (37) is neglected here.

In order for equation (44) to coincide with equations (6), the bracketed term must have a numerical value of 0.11. Unfortunately, values for $\mathrm{u}_{*}$ and $\mathrm{T}_{*}$ were not reported in references 7 to 10 nor is $\theta_{c}$ known for this experiment. However, it is possible to make reasonable estimates of $\mathbf{u}_{*}$ and $T_{*}$ from a general description of the experimental conditions and then compute the value of $\theta_{\mathbf{c}}$ which gives the best agreement between theory (eq. (44)) and experiment (eqs. (6)). It is found that a value for $\theta_{\mathrm{c}}$ of $0.87^{\circ}$ gives the best agreement when $T_{*}$ is estimated at $0.3^{\circ} \mathrm{C}$ and $u_{*}$ is computed to be $0.7 \mathrm{~m} / \mathrm{sec}$. (According to ref. 47 , the friction velocity $u_{*}$ can be estimated as approximately 12 percent of the mean windspeed for relatively rough terrains. This is how $u_{*}$ was estimated in this calculation.) The value of $0.87^{\circ}$ for $\theta_{c}$ is in good agreement with the values of $\theta_{c}$ which best fit the present theory to the data from the glider experiments of Beran and his collaborators (refs. 5, 6, and 11). See table II.

It is reported in references 7 to 10 that the accumulated excess attenuation does not increase linearly with source-receiver separation but instead appears to increase logarithmically, as in figure 2. In this experiment, the accumulated excess attenuation increases sharply with height to a height of about $200 \mathrm{~m}(600 \mathrm{ft})$. Beyond that, it increased approximately linearly with height. Since the slope of the curve of excess attenuation against height represents the excess attenuation coefficient, it is clear from figure 2 that the excess attenuation coefficient is greater near the surface than at greater altitudes. 
This experimental result is explained by noting that the turbulence structure constants, $\mathrm{C}_{\mathrm{V}}$ and $\mathrm{C}_{\mathrm{T}}$, both display an $\mathrm{r}^{-2 / 3}$ altitude dependence. (See eqs. (39).) If the temperature does not change very much over the altitude range of interest, then the accumulated excess attenuation, obtained by integrating equation (37) over the propagation path, will display a cube root source height dependence as in equation (40).

In figure 15, equation (40) is plotted against $h$ for a frequency of $1 \mathrm{kHz}$. The general shape of this curve is the same as in figure 2; it increases relatively steeply below about $200 \mathrm{~m}(600 \mathrm{ft})$ and then appears to increase nearly linearly with height above that. Thus, equation (40) predicts the general source height dependence which was observed experimentally.

The purpose of developing the excess attenuation theory in this report is to provide an improved scheme for correcting raw acoustic field data for atmospheric effects. The data in figure 16 are taken from reference 15 and represent a composite of the total measured attenuation coefficient as a function of frequency for several sets of field data accumulated under different average meteorological conditions and from different sources listed in reference 15 . The dashed line represents the theoretical curve for the classical plus molecular absorption and the solid line represents the theoretical curve for the

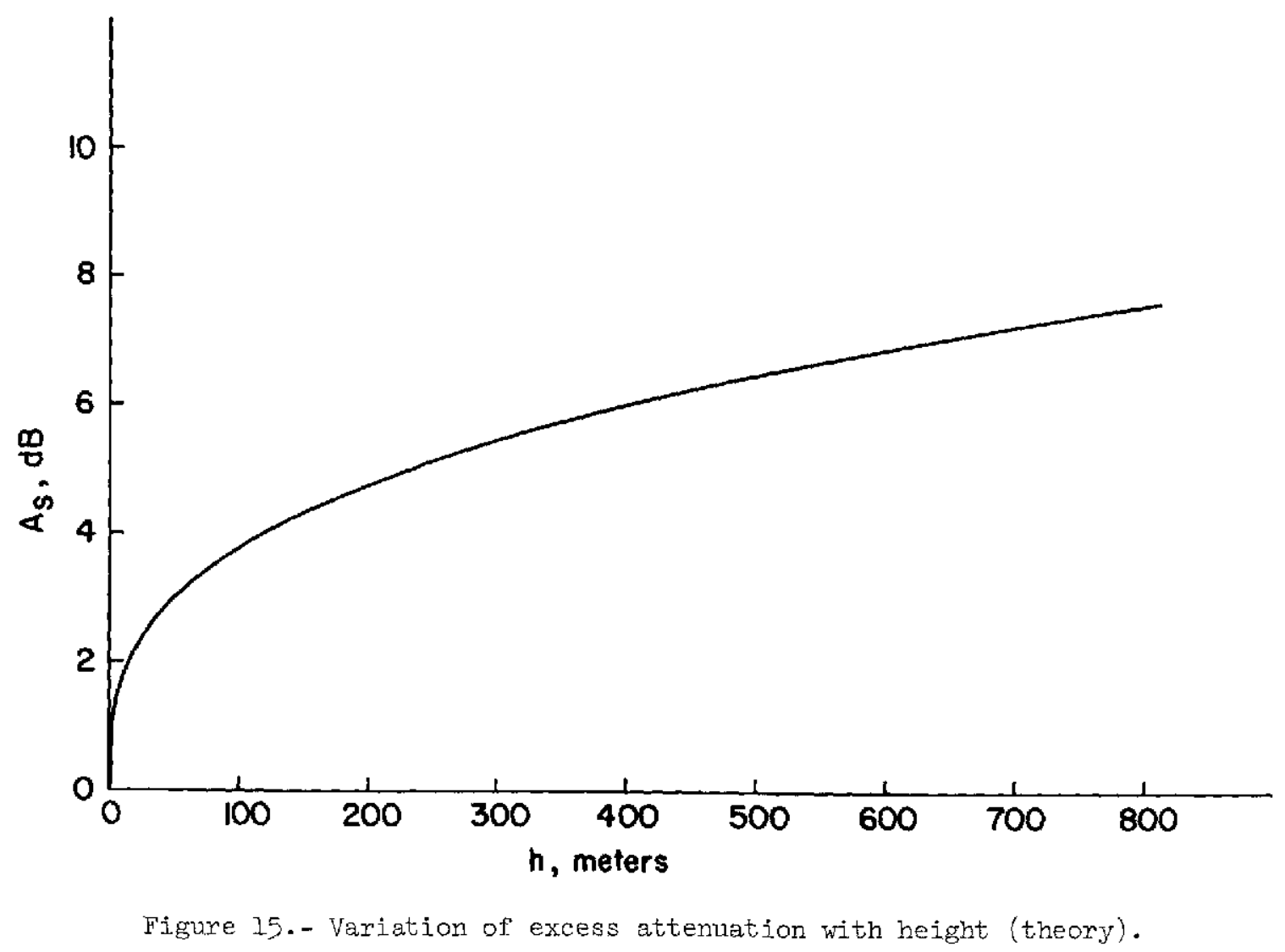



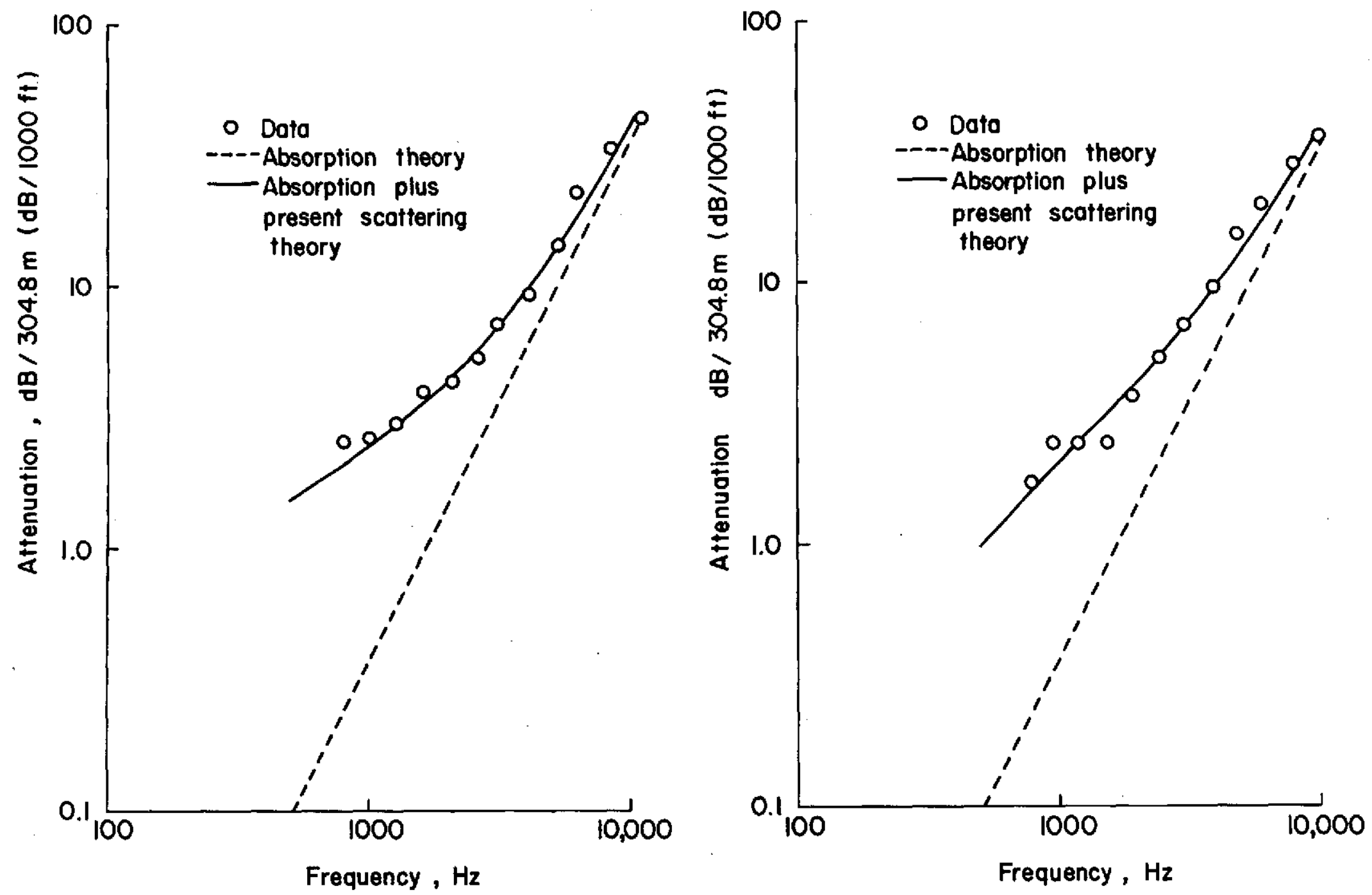
(a) $C_{V}=0.34 \mathrm{~m}^{2 / 3} / \mathrm{sec} ; C_{T}=0.17^{\circ} \mathrm{c} / \mathrm{m}^{\mathrm{I}} / 3$; $\theta_{c}=1.2^{\circ} ; \quad L=150 \mathrm{~m} ; \quad \overline{\mathrm{T}}=21.5^{\circ} \mathrm{C}$; and $\mathrm{H}=51.4$ percent.
(b) $C_{\mathrm{V}}=0.15 \mathrm{~m}^{2} / 3 / \mathrm{sec} ; C_{\mathrm{T}}=0.17^{\circ} \mathrm{c} / \mathrm{mI}^{2} / 3$; $\theta_{\mathrm{C}}=0.65^{\circ} ; L=72.7 \mathrm{~m} ; \quad T=5.5^{\circ} \mathrm{C}$; and $\mathrm{H}=60.6$ percent.



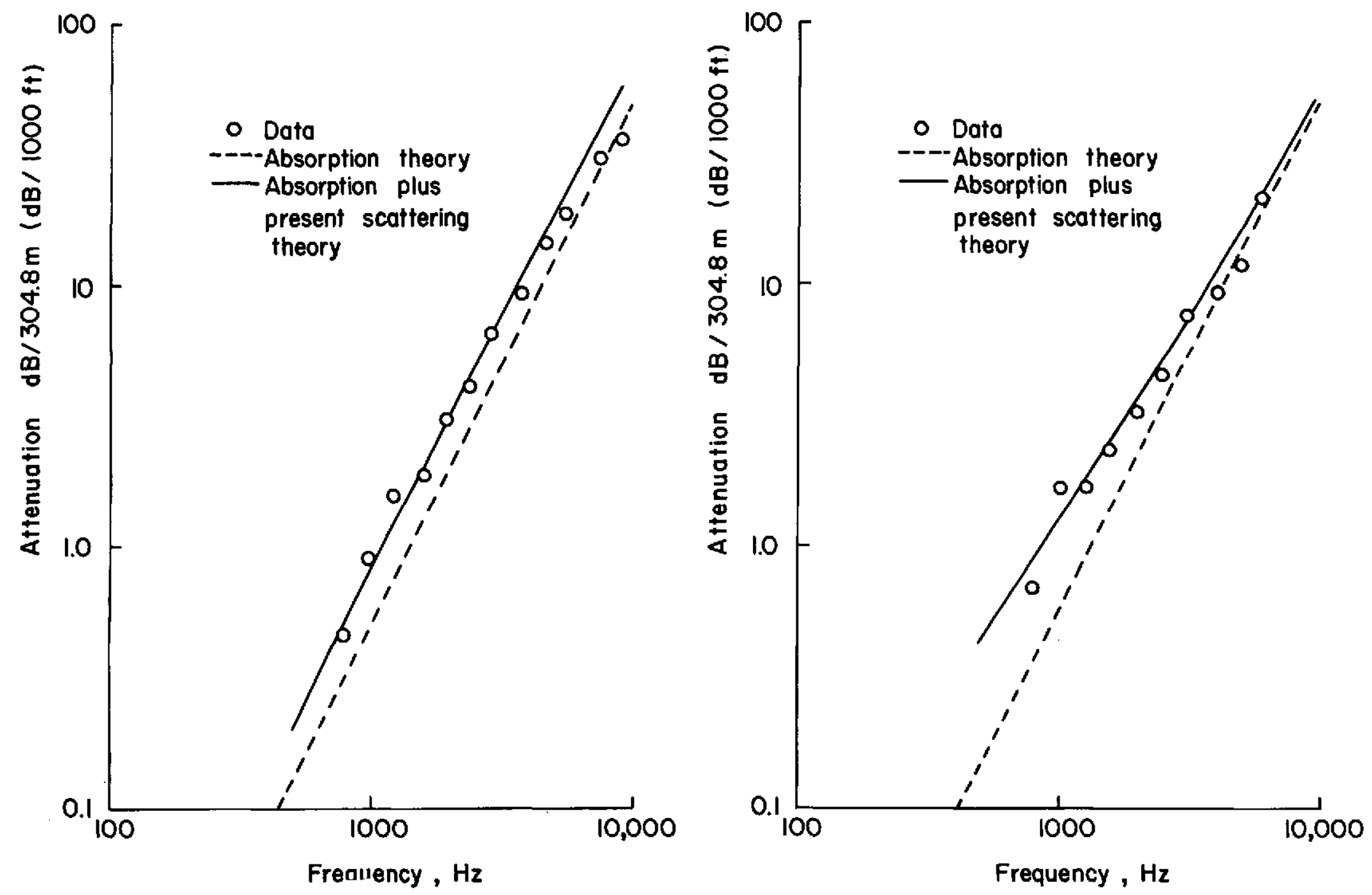
(c) $C_{V}=0.15 \mathrm{~m} 2 / 3 / \mathrm{sec} ; C_{\mathrm{T}}=0.17^{\circ} \mathrm{c} / \mathrm{m}^{\mathrm{I}} / 3$; $\theta_{c}=0.1^{\circ} ; L=7.6 \mathrm{~m} ; \quad T=8.61^{\circ} \mathrm{C}$; and $H=76$ percent.
(d) $C_{V}=0.15 \mathrm{~m}^{2} / 3 / \mathrm{sec} ; \quad C_{\mathrm{T}}=0.17^{\circ} \mathrm{C} / \mathrm{ml}^{1 / 3}$; $\theta_{C}=0.84^{\circ} ; \quad L=26.4 \mathrm{~m} ; \mathrm{T}=3.5^{\circ} \mathrm{C}$; and $H=88.3$ percent.




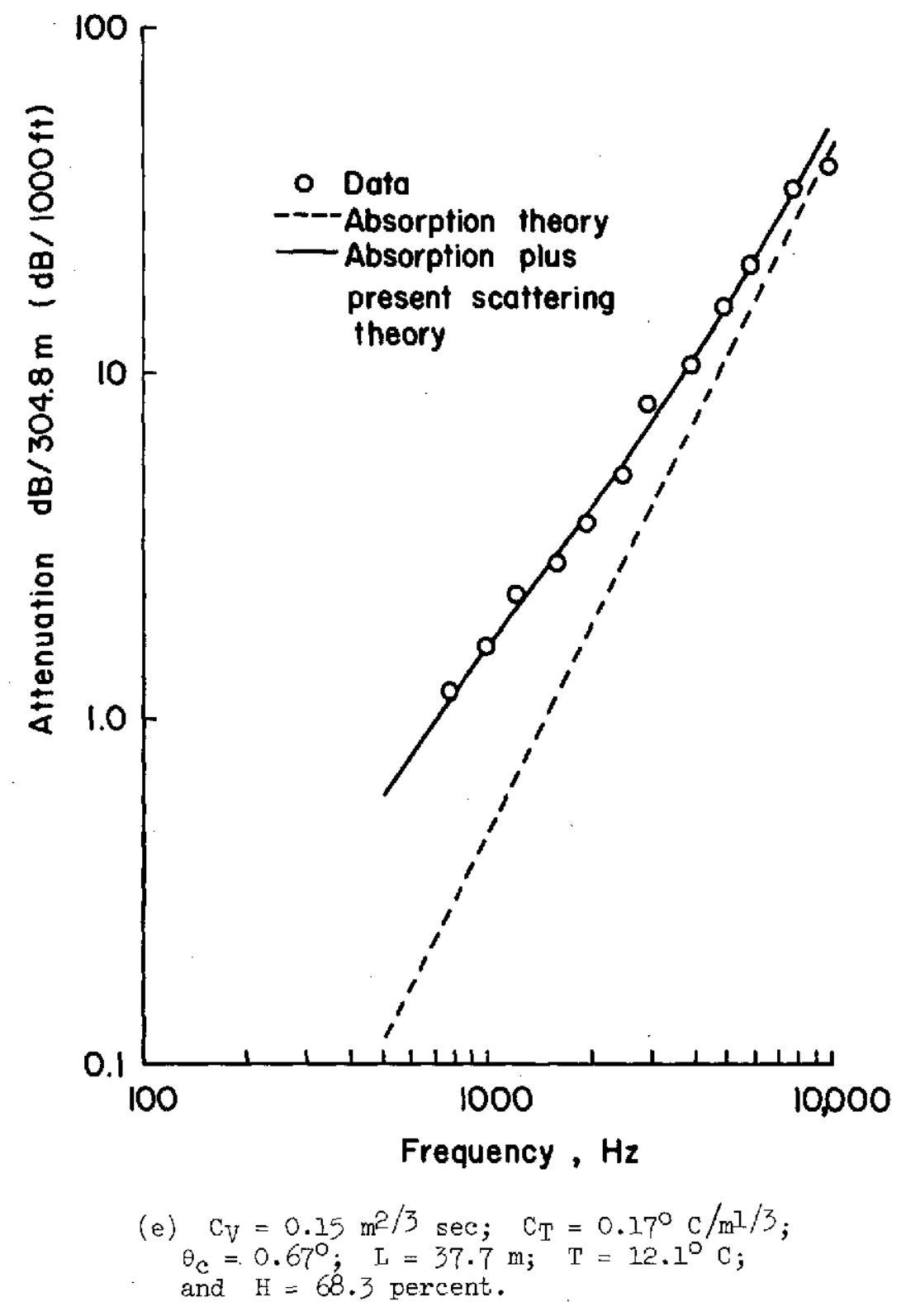

Figure 16.- Concluded. 
total attenuation, including both the absorption effects and the excess attenuation as it is described in the present theory.

Equation (37) expresses the excess attenuation coefficient in nepers/meter. In order to compare the present theory with the data in figure 16, this formula is expressed in $\mathrm{dB} / 1000 \mathrm{ft}$ as follows:

$$
\alpha_{\mathrm{s}}=602\left(\frac{\mathrm{C}_{\mathrm{V}}{ }^{2}}{\mathrm{c}^{2}}+0.136 \frac{\mathrm{C}_{\mathrm{T}}{ }^{2}}{\mathrm{~T}^{2}}\right) \mathrm{k}^{1 / 3}\left(\frac{\pi}{\mathrm{kL}}+\sin \frac{\theta_{\mathrm{c}}}{2}\right)^{-5 / 3}
$$

Measurements (unpublished) made by William Neff and John Gaynor of the National Oceanic and Atmospheric Administration Wave Propagation Laboratory indicate that $\mathrm{C}_{\mathbf{T}}$ takes on values in the range of a few hundredths to a few tenths ${ }^{o} \mathrm{~K} / \mathrm{m} 1 / 3$ in the lower few hundred meters of the atmosphere and the vertical component of $C_{V}$ ranges between $0.01 \mathrm{~m} / \mathrm{sec}$ and $0.5 \mathrm{~m}^{2} / 3 / \mathrm{sec}$ in the same region. For the purpose of making a comparison between theory and measurement, certain values within these ranges were assumed for the structure constants in equation (45) and the values of $L$ and $\theta_{c}$ which give the best fit of the data in figure 16 were determined by the method of least squares. The humidity and temperature data represent average measured values for these parameters and the turbulence parameters of best fit are given for each figure.

The molecular absorption coefficient is given in reference 5 as follows (with notational changes):

$$
\alpha_{m}=\frac{2 \mu_{\max } f}{c\left(\frac{\omega_{o}}{2 \pi f}+\frac{2 \pi f}{\omega_{o}}\right)}
$$

where $f$ is the acoustic frequency, $c$ is the speed of sound, $\omega_{0}$ is angular molecular relaxation frequency, and $\mu_{\max }$ is the maximum molecular absorption per wavelength, which can be computed by the following formula from reference 15:

$$
\mu_{\max }=(0.079)\left(\frac{2239}{\mathrm{~T}}\right)^{2} \frac{\mathrm{e}^{2239 / \mathrm{T}}}{\left(\mathrm{e}^{2239 / \mathrm{T}}-1\right)^{2}}
$$

where $T$ is in kelvins. The molecular relaxation frequency $\omega_{0}$ depends sensitively on the moisture content of the atmosphere and is given in reference 50 as 


$$
\frac{\omega_{\mathrm{O}}}{2 \pi}=1750 \mathrm{~h}^{\prime}+61400 \mathrm{~h}^{\prime}\left(\frac{1.12+10 \mathrm{~h}^{\dagger}}{10.4+10 \mathrm{~h}^{\dagger}}\right)
$$

where $h^{\prime}$ is the ratio of water vapor pressure to barometric pressure. According to reference $15, h^{\prime}$ is related to the relative humidity $\mathrm{H}$ in the range of $0^{\circ} \mathrm{C}$ to $25^{\circ} \mathrm{C}$ by the following relation:

$$
h^{\prime}=\frac{H}{(5.6)\left(30-\frac{T}{10}\right) e^{-0.0665 T}-1}
$$

Equations (46) to (49) can be used to calculate the molecular absorption of a given frequency from the temperature and relative humidity. The classical absorption can be computed by the following expression, which is based on a formula from reference 15 :

$$
\alpha_{\mathrm{c}}=4.77 \times 10^{-8}(1+0.001 \mathrm{~T}) \mathrm{f}^{2} \mathrm{~dB} / 1000 \mathrm{ft}
$$

where $\mathrm{T}$ is in ${ }^{\circ} \mathrm{C}$ and $\mathrm{f}$ is in $\mathrm{Hz}$.

Equations (46) to (50) were used to compute the straight-line absorption curves in figure 16 from temperature and humidity data. The curve for the total attenuation, which includes the excess attenuation as well as the absorption, is computed from equations (45) to (50). Note in figure 16, the classical plus molecular absorption theory does not adequately describe the total attenuation which is observed in typical field data, especially at the lower frequencies. When this absorption theory is augmented by the excess attenuation theory developed in this paper, the agreement between theory and measurement is much improved.

\section{DISCUSSION}

The Angle $\theta_{\mathrm{c}}$

Equations (25) can be expressed in terms of a minimum scattering angle $\theta_{0}$ which has the following value according to equation (14):

$$
\theta_{0}=2 \sin ^{-1} \frac{\lambda}{2 \bar{L}}
$$

where $I$ is the outer scale eddy size. This is the smallest angle through which sound of a given frequency will be scattered if equation (14) is valid. By equation (13), 


$$
\mathrm{K}_{\mathrm{o}}=2 \mathrm{k} \sin \frac{\theta_{\mathrm{O}}}{2}
$$

and equations (25) become

$$
\alpha_{\mathrm{S}}=0.455 \mathrm{k}^{1 / 3}\left(\frac{\mathrm{C}_{\mathrm{V}}^{2}}{\mathrm{c}^{2}}+0.136 \frac{\mathrm{C}_{\mathrm{T}}{ }^{2}}{\mathrm{~T}^{2}}\right) \sin ^{-5 / 3} \frac{\theta_{\mathrm{O}}}{2}
$$

If one assumes that the conventional Bragg condition (eq. (13)) is valid for all eddy sizes including the largest eddies, then one is forced to conclude that the minimum angle through which sound will scatter from a given eddy is inversely proportional to the acoustic frequency and thus, that the minimum scattering angle $\theta_{0}$ can be made arbitrarily small by increasing the frequency sufficiently. This assumption leads directly to equations (25) and the conclusion that the excess attenuation coefficient must display a square-law frequency dependence. Note, however, that if the minimum scattering angle in equation (53) were not a function of frequency, then the excess attenuation coefficient would display a cube-root frequency dependence. Benson et al. (refs. 7 to 10) did, in fact, report a cuberoot frequency dependence for the excess attenuation measured out of doors. Furthermore, Schmidt's wind-tunnel data (ref. 49) show a square-law frequency dependence for the excess attenuation coefficient under relatively mild turbulence conditions but the strength of the frequency dependence in his data decreases as the flow becomes more and more irregular and approaches a cube-root dependence asymptotically as the turbulence is increased. (See fig. 13.) These experimental observations led the author to hypothesize that in a relatively irregular turbulence field, the minimum scattering angle is in fact independent of the acoustic frequency and therefore that the conventional Bragg condition (eq. (13)) does not adequately describe the scattering of sound in such a medium. This hypothesis led to the empirical derivation of a modified Bragg condition (eq. (34)) which is expressed in terms of a frequency independent angle $\theta_{\mathrm{C}}$. The angle $\theta_{\mathrm{C}}$ can be interpreted physically by comparing equations (37) and (53); thus,

$$
\theta_{0}=2 \sin ^{-1}\left(\frac{\pi}{k \tilde{L}}+\sin \frac{\theta_{c}}{2}\right)
$$

As the acoustic frequency increases, the minimum scattering angle approaches $\theta_{\mathbf{c}}$ instead of zero. The angle $\theta_{c}$ therefore represents the smallest angle through which a sound wave will scatter in a medium for which the conventional Bragg condition breaks down. The fact that this angle is independent of frequency leads to the relatively mild frequency dependence which is predicted by equation (37) and which is observed experimentally. In figure 17, equations (51) and (54) are both plotted against wavelength to compare the two cases. 


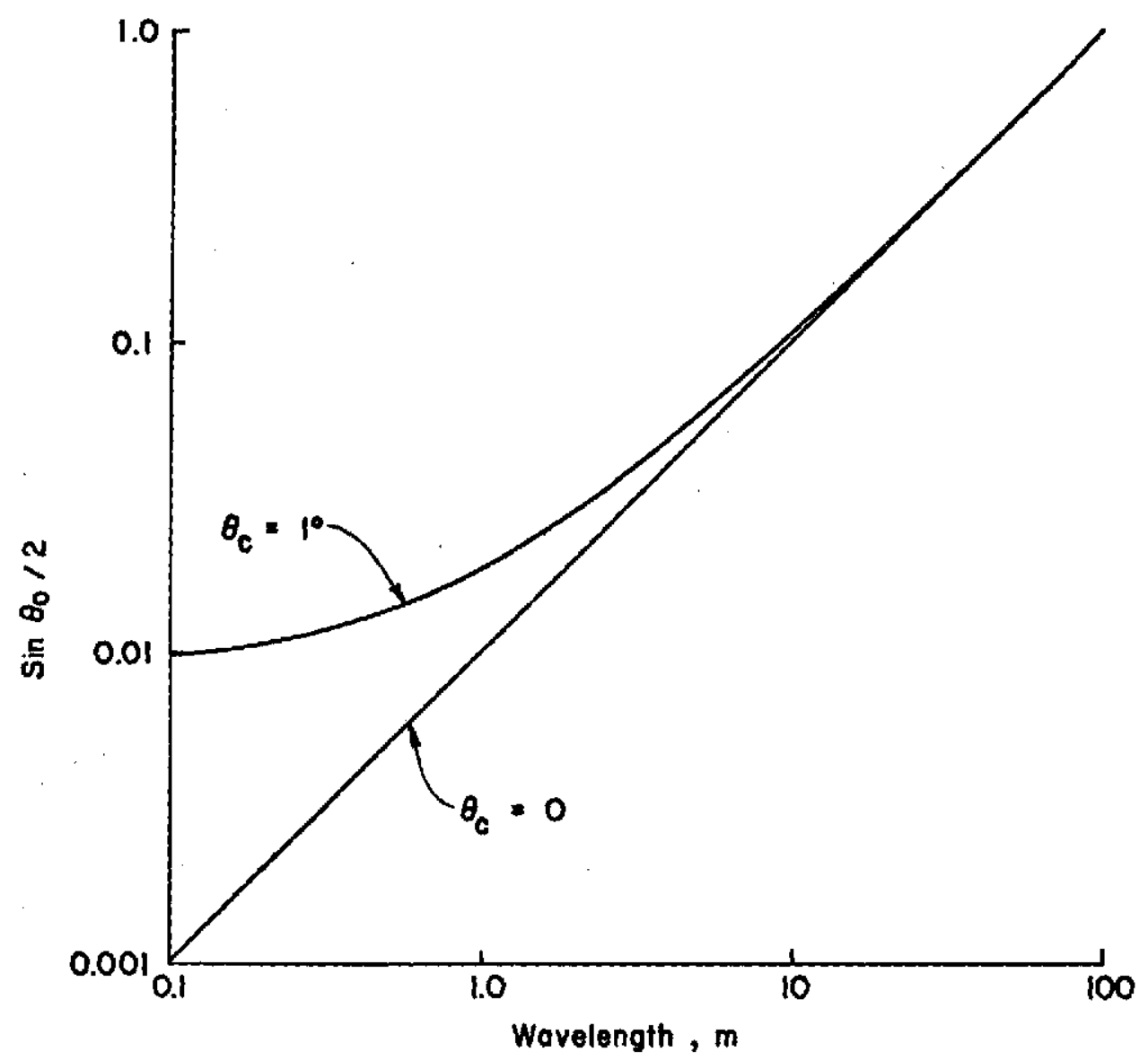

Figure 17.- Variation of $\sin \theta_{0} / 2$ with wavelength for $\dot{L}=50 \mathrm{~m} . \theta_{C}$ from equation (54).

One of the difficulties with equation (37) is that it is hard to determine either $\mathrm{L}$ or $\theta_{\mathrm{c}}$ by direct measurement. Note, however, that if either $\theta_{\mathrm{c}}$ or $\mathrm{L}$ were sufficiently large, the $\pi / \mathrm{kL}$ term could be neglected in equation (37) and then the excess attenuation coefficient would be essentially independent of $\mathrm{L}$. Although there are not enough available excess attenuation data to establish it conclusively, the mild frequency dependence which is consistently associated with outdoor excess attenuation measurements is a strong indication that for audio frequencies the $\sin \theta_{\mathrm{c}} / 2$ term in equation (37) does in fact dominate the $\pi / \mathrm{kL}$ term most of the time.

The $\theta_{\mathrm{c}}$ parameter, although difficult to determine experimentally, seems to vary over a fairly small range, judging from the little data that are currently available. Note, for example, that the $\theta_{\mathrm{c}}$ values which best fit Beran's glider experiment data to the present theory coincide very closely with the $\theta_{\mathrm{c}}$ values which best fit Benson's aircraft flyover data to the theory, even though the two sets of data were taken over 10 years apart, in different parts of the world, and at different times of the year. It is possible 
that the $\theta_{\mathfrak{c}}$ parameter depends in some way on the general terrain features and wind conditions, just as the friction velocity $u_{*}$ does. Perhaps as more excess attenuation data becomes available, it will be possible to tabulate typical values for $\theta_{c}$ corresponding to different types of terrain, just as had been done for the friction velocity.

\section{Limits of Applicability of Theory}

The calculations performed in this paper are based on the Kalistratova-TatarskiMonin representation for the scattering cross section (eq. (11)) and all the assumptions which were made in deriving this result are inherent in the present theory. Among these are the assumptions that the medium is inviscid, that the propagation process is adiabatic, and that the scattering volume is large enough for turbulent fluctuations in different parts of the volume to be statistically independent yet sufficiently small for the turbulence characteristics to remain unchanged during the passage of a sound wave. This last condition is called the frozen turbulence approximation and allows the temporal frequency depend ence of the spectral density functions to be ignored to first order. It is further assumed that the acoustic wavelengths are small compared with the outer scale of turbulence and that the mean flow velocity is small compared with the speed of sound. Finally, it is assumed that the Reynolds number is sufficiently large to allow the inertial subrange to occupy a large part of the turbulence spectrum.

Certain limitations originally ascribed to the cross-section formula by early workers in the field have since been shown by those workers and others to be unnecessarily restrictive. For example, Tatarski originally derived the cross-section formula for a homogeneous, isotropic medium, but he later demonstrated that the derivation also applies to a medium which is only locally homogeneous and isotropic. Kalistratova (ref. 36) originally proposed a lower limit on the angular acoustic frequency of $10^{4} \mathrm{sec}^{-1}$ for which the scattering cross section could be considered valid, but Gething (ref. 51) has shown that this lower frequency limit can be replaced by the assumption of a quasi-stationary turbulence field. This is just an extension of the frozen turbulence assumption which requires that the time derivatives of the correlation functions describing the turbulent fluctuations be small.

The scattering cross-section formula was derived by using a perturbation technique in which the density, pressure, entropy, and speed of sound were each represented as the sum of a zero-order term and small perturbation terms of higher order which are due to the acoustic disturbance. Terms of second order and higher were neglected.

Although there are several approximations and assumptions built into the excess attenuation theory and the general scattering theory upon which it is based, each of these approximations and assumptions can be expected to hold fairly well for the case of audio frequencies propagating in the lower atmosphere. The fact that there is agreement within experimental error between the scattering theory and experiment bears this fact out. 
Application of the Theory to the Solution of Aircraft

Noise Measurement Problems

NASA is interested in the excess attenuation problem because of the effects it has on the precision with which aircraft noise can be measured in the field. Current data acquisition systems are capable of measuring sound pressure levels with an absolute accuracy approaching $0.1 \mathrm{~dB}$, yet field data is seldom repeatable to this accuracy. The typical decibel spread in field aircraft noise data is generally more than an order of magnitude greater than the rated accuracy of the acquisition system, even when the sound source conditions are the same and the same absorption corrections are made for all the data.

One of the reasons for this relative lack of precision in "precision" aircraft field measurements is that not all the atmospheric effects are accounted for in reducing the data. Field noise data are generally supported only by measurements of mean meteorological parameters; the relatively rapid fluctuations in these parameters are not usually measured. Although this "dc" meteorological support may be sufficient to account for classical and molecular absorption effects, it is necessary to measure the "ac" components of the meteorological fields to account for the excess attenuation. According to observations discussed earlier, the excess attenuation generally has about the same effect on sound propagation as the classical plus molecular absorption; thus, for the purpose of supporting sound propagation measurements, it is just as important to measure fluctuations in the meteorological parameters as it is to measure their mean values.

Federal Aviation Regulations Part 36 (ref. 52) (designated FAR-36), which prescribes the noise standards and certification requirements by which the FAA aircraft noise abatement regulatory program is implemented, provides detailed instructions for normalizing raw acoustic data to reference mean meteorological conditions, but only one brief paragraph deals with turbulence effects. This paragraph prohibits the taking of aircraft flyover data for noise certification purposes when there are ". . . temperature inversions or anomalous wind conditions that would significantly affect the noise level . . . . The fact that there are no further guidelines in FAR-36 establishing which effects are significant (or even what is meant by "significant") has limited the application of this guideline to real-world measurement problems. In practical applications of FAR-36, the assumption is necessarily made that the effects of atmospheric dynamics are never significant, since a means for correcting raw acoustic data for these effects has never been developed.

There is clearly a need for quantitative guidelines dealing with the effects of turbulence on sound propagation to aid in the acquisition and analysis of aircraft noise certification data and field noise data in general. One possible approach would be to require that raw acoustic data be considered acceptable for conventional analysis only when 
the turbulence structure constants, $\mathrm{C}_{\mathrm{V}}$ and $\mathrm{C}_{\mathrm{T}}$, are below some established upper limit. These limiting values for $C_{V}$ and $C_{T}$ would define the levels of "significant" turbulence. Another possibility is to correct for the excess attenuation by normalizing the raw acoustic data to some set of reference turbulence conditions in the same way that absorption effects are accounted for by current data analysis techniques. Before any effective guidelines can be established for correcting raw noise data for the effects of atmospheric dynamics, more propagation measurements must be made which are supported by turbulence measurements.

Recent advances in remote turbulence detection techniques have made data on atmospheric dynamics much more readily available than in the past, when such data were only available from in situ point sensors. One of the most promising remote turbulence detection techniques is acoustic radar, which operates in much the same way as conventional electromagnetic radar systems do. A high-intensity pulse of sound is directed into the air and echoes are produced when some of the sound scatters from atmospheric turbulence. The intensity of the received echo is proportional to the turbulence structure constants, $C_{V}$ and $C_{T}$. The time of return for the echo is related through the speed of sound to the altitude of the turbulence scattering center which caused the echo. Thus, it is possible to profile the turbulence structure of the atmosphere along the entire propagation path of a sound wave originating from a point within the range of the radar (typically approximately $2 \mathrm{~km}$ ). References 53 to 59 deal with the construction and operation of acoustic radar systems.

No matter what measurement technique is used, it is clear that measurements of turbulence must be added to the spectrum of meteorological measurements which routinely support precision field acoustic data-acquisition activities. The attenuation due to scattering, which is responsible for much of the total atmospheric attenuation, can only be accounted for by monitoring the dynamics of the atmosphere.

\section{CONCLUSIONS}

The attenuation suffered by a sound wave propagating from an elevated source to the ground in excess of spherical spreading losses and classical and molecular absorption effects has been studied. The principal findings of this investigation are summarized as follows:

1. A nonnegligible attenuation in excess of classical and molecular absorption effects has been consistently reported in sound propagation experiments conducted out of doors.

2. The magnitude of the excess attenuation coefficient has been reported to fluctuate over a wide range of values, but under certain circumstances, it has just as great an effect on sound propagation as the classical and molecular absorption. 
3. Failure to correct for the excess attenuation contributes substantially to the relatively large standard deviation which usually characterizes outdoor sound propagation measurements.

4. Although there are other mechanisms which also play a role, the scattering of sound by turbulent density and momentum fluctuations is a major cause of the excess attenuation for the case of air-to-ground propagation.

5. The scattering of sound by turbulence can be interpreted as a Bragg diffraction process if the conventional Bragg condition is modified by introducing a minimum scattering angle which depends only on properties of the medium and is independent of the acoustic frequency.

6. The formula for the coefficient of attenuation due to scattering in a turbulent medium $\alpha_{\mathrm{S}}$ is

$$
\alpha_{s}=0.455\left(\frac{C_{V}^{2}}{c^{2}}+0.136 \frac{C_{T}^{2}}{T^{2}}\right) k^{1 / 3}\left(\frac{\pi}{k L}+\sin \frac{\theta_{c}}{2}\right)^{-5 / 3}
$$

where

$\mathrm{C}_{\mathrm{T}} \equiv$ temperature structure constant

$\mathrm{C}_{\mathrm{V}} \equiv$ wind structure constant

c $\quad \equiv \quad$ speed of sound

$\mathrm{k} \quad \equiv \quad$ acoustic wave number

$\mathrm{L} \quad \equiv \quad$ scale of turbulence in direction of propagation

$\mathrm{T} \equiv$ temperature

$\theta_{\mathrm{c}} \equiv$ difference between true scattering angle and Bragg scattering angle

7. The frequency dependence of the excess attenuation lies between a square-law dependence and a cube root dependence. For a homogeneous isotropic medium, the excess attenuation depends on the square of the frequency, but for a medium with a more irregular outer scale, the frequency dependence is much weaker. In such a medium, the 
frequency ( $f$ ) dependence is very nearly $\mathrm{f} 1 / 3$ when the outer scale is large compared with half an acoustic wavelength (the general case for audio frequencies in the atmosphere).

8. The reported nonlinear altitude dependence of the excess attenuation is attributed to the decrease in atmospheric turbulence intensity with increasing altitude.

9. The correlation which has been reported between the magnitude of the excess attenuation and windspeed gradients is due to wind-shear-induced energy input into the turbulence spectrum.

10. A primary contributor to the excess attenuation observed under calm conditions (low windspeed and small windspeed gradients) is the turbulence induced by temperature fluctuations in the atmosphere.

11. In meteorological measurements which routinely support outdoor acoustic data acquisition activities, only mean values for the meteorological parameters are recorded. It is recommended that this meteorological data be augmented with turbulence measurements in order to account for the attenuation due to scattering. Only by accounting for the effects of atmospheric dynamics can the accuracy of outdoor sound propagation measurements be made to approach the rated accuracy of state-of-the-art data acquisition systems.

Langley Research Center, National Aeronautics and Space Administration, Hampton, Va., December 19, 1974. 


\section{APPENDIX A}

\section{EVALUATION OF THE EXCESS ATTENUATION INTEGRAL}

The excess attenuation coefficient is expressed as an integral in equation (20). This integral is evaluated

$$
\alpha_{\mathrm{S}}=\int_{0}^{\pi} 4 \pi^{2} \mathrm{k}^{4} \cos ^{2} \theta\left[\frac{\mathrm{E}(\mathrm{K})}{\mathrm{c}^{2}} \cos ^{2} \frac{\theta}{2}+\frac{\Phi(\mathrm{K})}{4 \mathrm{~T}^{2}}\right] \sin \theta \mathrm{d} \theta
$$

Equation (A1) can be expressed as follows:

$$
\alpha_{s}=4 \pi^{2} k^{4}\left(\frac{I_{1}}{c^{2}}+\frac{I_{2}}{4 T^{2}}\right)
$$

where

$$
\mathrm{I}_{1}=\int_{0}^{\pi} \cos ^{2} \theta \cos ^{2} \frac{\theta}{2} \sin \theta \mathrm{E}(\mathrm{K}) \mathrm{d} \theta
$$

and

$$
I_{2}=\int_{0}^{\pi} \cos ^{2} \theta \sin \theta \Phi(K) d \theta
$$

Assume that $\mathrm{K}$ and $\theta$ are related by the Bragg condition

$$
\mathrm{K}=2 \mathrm{k} \sin \frac{\theta}{2}
$$

Express the spectral density functions, $\mathrm{E}(\mathrm{K})$ and $\Phi(\mathrm{K})$, as in equations (21) and introduce the following change of variables:

$$
y=\sin \frac{\theta}{2}
$$


Then, by equation (A5),

$$
\mathrm{K}=2 \mathrm{ky}
$$

and equations (A3) and (A4) become

$$
\begin{aligned}
\mathrm{I}_{1}= & 4 \xi^{\prime}(2 \mathrm{k})^{\mathrm{m}} \int_{0}^{\mathrm{y}} \mathrm{o}\left(\mathrm{y}-5 \mathrm{y}^{3}+8 \mathrm{y}^{5}-4 \mathrm{y}^{7}\right) \mathrm{y}^{\mathrm{m}} \mathrm{dy} \\
& +4 \xi(2 \mathrm{k})^{\mathrm{n}} \int_{\mathrm{y}_{\mathrm{o}}}^{1}\left(\mathrm{y}-5 \mathrm{y}^{3}+8 \mathrm{y}^{5}-4 \mathrm{y}^{7}\right)_{\mathrm{y}^{\mathrm{n}} \mathrm{dy}} \\
\mathrm{I}_{2}= & 4 \eta^{\prime}(2 \mathrm{k})^{\mathrm{m}} \int_{0}^{\mathrm{y}}\left(\mathrm{y}-4 \mathrm{y}^{3}+4 \mathrm{y}^{5}\right) \mathrm{y}^{\mathrm{m}} \mathrm{dy} \\
& +4 \eta(2 \mathrm{k})^{\mathrm{n}} \int_{\mathrm{y}_{\mathrm{o}}}^{1}\left(\mathrm{y}-4 \mathrm{y}^{3}+4 \mathrm{y}^{5}\right) \mathrm{y}^{\mathrm{n}} \mathrm{dy}
\end{aligned}
$$

where

$$
\mathrm{y}_{\mathrm{o}}=\frac{\mathrm{K}_{\mathrm{O}}}{2 \mathrm{k}}
$$

The integrals in equations (A8) and (A9) can be evaluated as follows:

$$
\begin{aligned}
I_{1}= & 4 \xi^{\prime}(2 \mathrm{k}) \mathrm{m}\left(\frac{\mathrm{y}_{\mathrm{o}}^{\mathrm{m}+2}}{\mathrm{~m}+2}-\frac{5 \mathrm{y}_{\mathrm{o}}^{\mathrm{m}+4}}{\mathrm{~m}+4}+\frac{8 \mathrm{y}_{\mathrm{o}}^{\mathrm{m}+6}}{\mathrm{~m}+6}-\frac{4 \mathrm{y}_{\mathrm{o}}^{\mathrm{m}+8}}{\mathrm{~m}+8}\right) \\
& +4 \xi(2 \mathrm{k})^{\mathrm{n}}\left[\frac{1-\mathrm{y}_{\mathrm{o}}^{\mathrm{n}+2}}{\mathrm{n}+2}-\frac{5\left(1-\mathrm{y}_{\mathrm{o}}^{\mathrm{n}+4}\right)}{\mathrm{n}+4}+\frac{8\left(1-\mathrm{y}_{\mathrm{o}}^{\mathrm{n}+6}\right)}{\mathrm{n}+6}-\frac{4\left(1-\mathrm{y}_{\mathrm{o}}^{\mathrm{n}+8}\right)}{\mathrm{n}+8}\right] \\
\mathrm{I}_{2}= & 4 \eta^{\prime}(2 \mathrm{k})^{\mathrm{m}}\left(\frac{\mathrm{y}_{\mathrm{o}}^{\mathrm{m}+2}}{\mathrm{~m}+2}-\frac{4 \mathrm{y}_{\mathrm{o}}^{\mathrm{m}+4}}{\mathrm{~m}+4}+\frac{4 \mathrm{y}_{\mathrm{o}}^{\mathrm{m}+6}}{\mathrm{~m}+6}\right) \\
& +4 \eta(2 \mathrm{k})^{\mathrm{n}}\left[\frac{1-\mathrm{y}_{\mathrm{o}}^{\mathrm{n}+2}}{\mathrm{n}+2}-\frac{4\left(1-\mathrm{y}_{\mathrm{o}}^{\mathrm{n}+4}\right)}{\mathrm{n}+4}+\frac{4\left(1-\mathrm{y}_{\mathrm{o}}^{\mathrm{n}+6}\right)}{\mathrm{n}+6}\right]
\end{aligned}
$$




\section{APPENDIX A - Concluded}

For audio wavelengths in the atmosphere, $y_{0} \ll 1$. Therefore, those terms in equations (A11) and (A12) in which $y_{0}$ is raised to a nonnegative exponent are negligibly small compared with those terms in which $y_{0}$ is raised to a negative exponent. If, for example, $\mathrm{m}>-2$ and $-4<\mathrm{n}<-2$, and assuming $\xi^{\dagger} \lesssim \xi$ and $\eta^{\prime} \lesssim \eta$, equations (A11) and (A12) reduce to the following:

$$
\begin{aligned}
& I_{1}=\frac{4 \xi(2 k)^{n} y_{o}^{n+2}}{-(n+2)}=\frac{\xi k^{-2} K_{o}^{n+2}}{-(n+2)} \\
& I_{2}=\frac{4 \eta(2 k)^{n_{1} y_{o}^{n+2}}}{-(n+2)}=\frac{\eta k^{-2} K_{o}^{n+2}}{-(n+2)}
\end{aligned}
$$

Insert equations (A13) and (A14) into equation (A2) to obtain

$$
\alpha_{S}=\frac{4 \pi^{2} k^{2} K_{o}^{n+2}}{-(n+2)}\left(\frac{\xi}{c^{2}}+\frac{\eta}{4 T^{2}}\right)
$$

Equation (A15) is identical to equation (22).

This result is valid for $\mathrm{m}>-2$ and for $-4<\mathrm{n}<-2$, provided that $\mathrm{y}_{\mathrm{O}}=\frac{\mathrm{K}_{\mathrm{O}}}{2 \mathrm{k}} \ll 1$. These relations are, in fact, very mild constraints which are almost always met. For example, if the spectral density functions are to represent something physical, they must be nonsingular at the origin; this means that $m$ in equations (21) must be nonnegative. The necessary condition that $m$ be greater than -2 is therefore met for physically realizable spectral density functions. The value of $n$ is known to be $-\frac{11}{3}$ in the inertial subrange and since this value lies between -4 and -2 , the constraint on $n$ is satisfied.

Finally, the condition that $\frac{\mathrm{K}_{\mathrm{O}}}{2 \mathrm{k}} \ll 1$ requires that the outer scale eddies be large compared with half an acoustic wavelength. This condition is almost always met for the case of audio wavelengths in the atmosphere. 


\section{APPENDDX B}

\section{A REPRESENTATION OF THE STRUCTURE CONSTANTS IN TERMS OF FLUCTUATIONS IN WIND AND TEMPERATURE}

The turbulence structure constants, $C_{V}$ and $C_{T}$, can be expressed in terms of fluctiations in the windspeed and the temperature, respectively, by applying a method used by Strohbehn (ref. 46) to the case of a homogeneous and isotropic spectrum of turbulence for which equations (21) describe the spectral density functions. Begin by considering a covariance function $\mathrm{D}(\overrightarrow{\mathrm{r}})$ which describes a random function $\mathrm{F}(\overrightarrow{\mathrm{r}})$. By definition,

$$
D_{F}(\vec{r})=\overline{\left[F\left(\vec{r}_{1}\right)-\overrightarrow{F\left(\vec{r}_{1}\right)}\right]\left[F\left(\vec{r}_{1}+\vec{r}\right)-\overline{F\left(\vec{r}_{1}+\vec{r}\right)}\right.}
$$

where the bar represents an average value, $\vec{r}_{1}$ is a position vector, and $\vec{r}$ is a displacement vector. Define $\Delta F$ as the fluctuation of $F$ about its average value:

$$
\Delta F=F\left(\vec{r}_{1}\right)-\overline{F\left(\mathbf{r}_{1}\right)}
$$

By equations (B1) and (B2),

$$
\lim _{r \rightarrow 0} D_{F}(\vec{r})=\overline{(\Delta F)^{2}}
$$

The covariance function describing wind fluctuations (which reduces to a simple correlation function for zero mean wind) is related to the spectral density function for wind fluctuations $\mathbf{E}(\mathrm{K})$ by a Fourier transformation. That is, if the random function $v(\vec{r})$ represents the fluctuating component of the windspeed, then $D_{V}(\vec{r})$ and $E(\vec{K})$ are Fourier transform pairs

$$
E(\vec{K})=\frac{1}{(2 \pi)^{3}} \iint_{-\infty}^{+\infty} \int_{v}(\vec{r}) e^{-i \vec{K} \cdot \vec{r}} d \vec{r}
$$

and

$$
D_{V}(\vec{r})=\iiint_{-\infty}^{+\infty} E(\vec{K}) e^{i \vec{K} \cdot \vec{r}} \overrightarrow{d K}
$$


If $\mathrm{v}(\vec{r})$ is statistically isotropic, the vector notation can be dropped in equations (B4) and (B5), spherical coordinates can be-introduced, and the angular integration can be carried out explicitly, with the following result:

$$
\begin{aligned}
& E(K)=\frac{1}{2 \pi^{2} K} \int_{0}^{\infty} r D_{v}(r) \sin (K r) d r \\
& D_{v}(r)=\frac{4 \pi}{r} \int_{0}^{\infty} K E(K) \sin (K r) d K
\end{aligned}
$$

By equations (B3) and (B7),

$$
\begin{aligned}
& \overline{(\Delta v)^{2}}=\lim _{r \rightarrow 0} \frac{4 \pi}{r} \int_{0}^{\infty} K E(K) \sin (K r) d r \\
& \overline{(\Delta v)^{2}}=4 \pi \int_{0}^{\infty} K^{2} E(K) d K
\end{aligned}
$$

Insert equation (21a) into equation (B9) to obtain

$$
\overline{(\Delta v)^{2}}=4 \pi \int_{0}^{K_{0}} \xi^{\prime} K^{m+2} d K+4 \pi \int_{K_{0}}^{\infty} \xi K^{n+2} d K
$$

After integrating,

$$
\overline{(\Delta v)^{2}}=4 \pi\left(\frac{\xi^{4} K_{0}^{m+3}}{m+3}-\frac{\xi K_{o}^{n+3}}{n+3}\right)
$$

By equation (24), $\mathrm{n}=-\frac{11}{3}$ and equation (B11) becomes

$$
\overline{\Delta v^{2}}=4 \pi\left(\frac{\xi^{\prime} \mathrm{K}_{\mathrm{o}}^{\mathrm{m}+3}}{\mathrm{~m}+3}+\frac{3}{2} \xi \mathrm{K}_{\mathrm{o}}^{-2 / 3}\right)
$$


In the atmosphere $K_{0}$ is generally small compared with one and, as it is argued in appendix $A, m$ must be nonnegative. Therefore, the first term inside the parentheses in equation (B12) is small compared with the second and equation (B12) becomes

$$
\overline{\Delta \mathrm{v}^{2}} \approx 6 \pi \xi \mathrm{K}_{\mathrm{o}}^{-2 / 3}
$$

Let $\gamma=\xi \mathrm{K}_{\mathrm{o}}^{-2 / 3}$. Then, by equation (B13),

$$
\gamma=\overline{\frac{\Delta v^{2}}{6 \pi}}
$$

By equations (21) (for $\mathrm{K}>\mathrm{K}_{\mathrm{O}}$ ),

$$
\mathrm{E}(\mathrm{K})=\gamma \mathrm{K}_{\mathrm{O}}^{2 / 3} \mathrm{~K}^{-11 / 3}
$$

or

$$
E(K)=\overline{\frac{\Delta v^{2}}{6 \pi}} K_{o}^{2 / 3} K^{-11 / 3} \quad\left(K>K_{o}\right)
$$

Equation (B15) is equivalent to equation (23a). Therefore,

$$
\frac{11 \Gamma\left(\frac{8}{3}\right) \sin \frac{\pi}{3}}{24 \pi^{2}} \mathrm{C}_{V^{2}}=\frac{\overline{(\Delta v)^{2}} K_{\mathrm{O}}^{2 / 3}}{6 \pi}
$$

or

$$
\mathrm{C}_{\mathrm{V}}{ }^{2}=\frac{4 \pi \mathrm{K}_{\mathrm{o}}^{2 / 3} \overline{(\Delta v)^{2}}}{11 \Gamma\left(\frac{8}{3}\right) \sin \frac{\pi}{3}}
$$

This is equation (26a). The transform relationship between the covariance function describing temperature fluctuations and the temperature structure density function can be exploited in a similar manner to express $\mathrm{C}_{\mathrm{T}}{ }^{2}$ in terms of $\overline{(\Delta \mathrm{T})^{2}}$ with the following result:

$$
\mathrm{C}_{\mathrm{T}}{ }^{2}=\frac{2 \pi \mathrm{K}_{\mathrm{O}}^{2 / 3} \overline{(\Delta \mathrm{T})^{2}}}{3 \Gamma\left(\frac{8}{3}\right) \sin \frac{\pi}{3}}
$$




\section{REFERENCES}

1. Anon.: Standard Values of Atmospheric Absorption as a Function of Temperature and Humidity for Use in Evaluating Aircraft Flyover Noise. ARP 866, Soc. Automot. Eng., Aug. 31, 1964.

2. Kneser, Hans O.: The Interpretation of the Anomalous Sound-Absorption in Air and Oxygen in Terms of Molecular Collisions. J. Acoust. Soc. Amer., vol. V, no. 2, Oct. 1933, pp. 122-126.

3. Harris, Cyril M.: Absorption of Sound in Air in the Audio-Frequency Range. J. Acoust. Soc. Amer., vol. 35, no. 1, Jan. 1963, pp. 11-17.

4. Knudsen, V. O.: Effects of Humidity Upon the Absorption of Sound in a Room and a Determination of the Coefficients of Absorption of Sound in Air. J. Acoust. Soc. Amer., vol. 3, no. 1, pt. 1, July 1931, pp. 126-138.

5. Beran, D. W.; Reynolds, R. M.; and Gething, J. T.: Sound Attenuation in the Free Atmosphere. Publ. No. 17, Project EAR, Rep. No. V, Meteorol. Dep., Univ. of Melbourne, Dec. 1970.

6. Beran, Donald W.: Turbulence Detection. Ph. D. Thesis, Univ. of Melbourne, July 1970.

7. Benson, R. W.; and Karplus, H. B.: Sound Propagation Near the Earth's Surface as Influenced by Weather Conditions. WADC Tech. Rep. 57-353, Part I, U.S. Air Force, Mar. 1958. (Available from DDC as AD 130 793.)

8. Burkhard, M. D.; Karplus, H. B.; and Sabine, H. J.: Sound Propagation Near the Earth's Surface as Influenced by Weather Conditions. WADC Tech. Rep. 57-353, Part II, U.S. Air Force, Dec. 1960.

9. Sabine, H. J.; Raelson, V. J.; and Burkhard, M. D.: Sound Propagation Near the Earth's Surface as Influenced by Weather Conditions. WADC Tech. Rep. 57-353, Part III, U.S. Air Force, Jan. 1961.

10. Sabine, H. J.: Sound Propagation Near the Earth's Surface as Influenced by Weather Conditions. WADC Tech. Rep. 57-353, Part IV, U.S. Air Force, Jan. 1961.

11. Beran, D. W.; and Gething, J. T.: Use of a Sailplane in Measuring Acoustic Attenuation in the Atmosphere. Aero-Revue, Issue 2, Feb. 1972, pp. 93-95.

12. Knudsen, Vern O.: The Absorption of Sound in Air, in Oxygen, and in Nitrogen Effects of Humidity and Temperature. J. Acoust. Soc. Amer., vol. V, no. 2, Oct. 1933, pp. 112-121. 
13. Delsasso, L. P.; and Leonard, R. W.: The Attenuation of Sound in the Atmosphere. Summary Report U.S. Air Force Contract W-28-099-AC-228, Univ. of California, Feb. 25, 1953.

14. Coles, G. M.: Atmospheric Absorption of Noise. Aerodynamic Noise, Univ. of Toronto Press, c.1969, pp. 209-227.

15. Evans, L. B.; and Sutherland, L. C.: Absorption of Sound in Air. WR 70-14 (Contract DAH C04-69C 0088), Wyle Labs., July 1970.

16. Ingard, Uno; and Oleson, Stanley K.: Measurements of Sound Attenuation in the Atmosphere. AFCRL-TR 60-431, U.S. Air Force, Nov. 4, 1960.

17. Ingard, Uno: The Physics of Outdoor Sound. Proceedings of the Fourth Annual National Noise Abatement Symposium, Vol. 4, Oct. 23-24, 1953, pp. 11-25.

18. Thompson, J. R.; and Shapiro, N.: The Effect of Temperature Inversions on Flyover Noise Measurements. Paper presented at Symposium on Atmospheric Acoustics and Noise Propagation (Gaithersburg, Md.), Sept. 1972.

19. Ingard, Uno: A Review of the Influence of Meteorological Conditions on Sound Progation. J. Acoust. Soc. Amer., vol.25, no. 3, May 1953, pp. 405-411.

20. Henley, David C.; and Hoidale, Glenn B.: Attenuation and Dispersion of Acoustic Energy by Atmospheric Dust. J. Acoust. Soc. Amer., vol. 54, no. 2, Aug. 1973, pp. 437-445.

21. Zink, William; Delsasso, L. P.; and Cox, Charlotte Jean: Attenuation and Dispersion of Sound by Solid Particles Suspended in a Gas. Contract 51-0796, Dept. Phys., Univ, of California, June 26, 1957.

22. Cole, John E., III; and Dobbins, Richard A.: Propagation of Sound Through Atmospheric Fog. Atmos. Sci., vol. 27, no. 3, May 1970, pp. 426-434.

23. Harris, Cyril M.: Absorption of Sound in Air in the Audio-Frequency Range. J. Acoust. Soc. Amer., vol. 35, no. 1, Jan. 1963, pp. 11-17.

24. Harris, Cyril M.: On the Absorption of Sound in Humid Air at Reduced Pressures. J. Acoust. Soc. Amer., vol. 43, no. 3, Mar. 1968, pp. 530 532.

25. Tyndall, John: The Science of Sound. New York, Phil. Lib., Inc. (New York), c.1964.

26. Lindsay, R. B.: Compressional Wave Front Propagation Through a Simple Vortex. J. Acoust. Soc. Amer., vol. 20, no. 2, Mar. 1948, pp. 89-94.

27. Müller, Ernst-August; and Matschat, Klaus R.: The Scattering of Sound by a Single Vortex and by Turbulence. AFOSR TN 59-337, ASTIA Doc. AD 213 658, U.S. Air Force, Jan, 1959. 
28. Obukov, A. M.: On the Scattering of Sound in a Turbulent Flow. Doklady Acad. Nauk, S.S.S.R., vol. 30, 1941 ; p. 611.

29. Obukov, A. M.: On Propagation of Sound Waves in Eddying Flow. Compt. Rend. Acad. Sci. URSS, vol. 39 , no. 2 , 1943 , pp. 46-48.

30. Batchelor, G. K.: Wave Scattering Due to Turbulence. Symposium on Naval Hydrodynamics, Publ. 515, Nat. Acad. Sci. - Nat. Res. Counc. 1957, pp. 409-430.

31. Kraichnan, Robert H.: The Scattering of Sound in a Turbulent Medium. J, Acoust. Soc. Amer, vol. 25, no. 6, Nov. 1953, pp. 1096-1104. (Errata. J. Acoust. Soc. Amer., vol. 28, no. 2, Mar. 1956, p. 314.)

32. Pekeris, C. L.: Note on the Scattering of Radiation in an Inhomogeneous Medium. Phys. Rev., vol. 71, no. 4, Feb. 1947, pp. 268-269.

33. Blokhintzev, D.: The Propagation of Sound in an Inhomogeneous and Moving Medium I. Part I. J. Acoust. Soc. Amer., vol. 18, no. 2, Oct. 1946, pp. 322-328.

34. Blokhintzev, D.: The Propagation of Sound in an Inhomogeneous and Moving Medium II. Part II. J. Acoust. Soc. Amer., vol. 18, no. 2, Oct. 1946, pp. 329-334.

35. Tatarski, V. I. (R. A. Silverman, transl.): Wave Propagation in a Turbulent Medium. McGraw-Hill Book Co., Inc., 1961.

36. Kallistratova, M. A.: Experimental Investigation of Sound Wave Scattering in the Atmosphere. FTD-TT-63-447, U.S. Air Force, June 1963. (Available from DDC as $A D 412821$.)

37. Kallistratova, M. A.; and Tatarskii, V. I.: Accounting for Wind Turbulence in the Calculation of Sound Scattering in the Atmosphere. Soviet Physics - Acoustics, vol. 6, no. 4, Apr.-June 1961, pp. 503-505.

38. Monin, A. S.: Characteristics of the Scattering of Sound in a Turbulent Atmosphere. Soviet Physics - Acoustics, vol. 7, no. 4, Apr.-June 1962, pp. 370-373.

39. Lighthill, M. J.: On the Energy Scattered From the Interaction of Turbulence With Sound or Shock Waves. Proc. Cambridge Phil. Soc., vol. 49, pt. 3, Cambridge Univ. Press, July 1953, pp. 531-555.

40. Lighthill, M. J.: On Sound Generated Aerodynamically. I. General Theory. Proc. Roy. Soc. (London), ser. A, vol, 211, no. 1107, Mar. 20, 1952, pp. 564-591.

41. Chernov, Lev A. (R. A. Silverman, transl.): Wave Propagation in a Random Medium. McGraw-Hill Book Co., Inc., c.1960.

42. Buell, C. Eugene: Sound Propagation Prediction. Contract No. NAS 8-11348, Kaman Nuclear, [1966]. (Available as NASA CR-69793.) 
43. Ingard, Uno; and Wiener, Francis M.: On the Scattering of Sound by Atmospheric Turbulence. J. Acoust. Soc. Amer., vol. 30, no. 7, July 1958, p. 670.

44. Baerg, William: Measurements of the Scattering of Sound From Turbulence. Ph. D. Thesis, Stanford Univ., Nov. 1964.

45. Sheih, C. M.; Tennekes, H.; and Lumley, J. L.: Airborne Hot-Wire Measurements of the Small-Scale Structure of Atmospheric Turbulence. Phys. Fluids, vol. 14, no. 2, Feb. 1971, pp. 201-215.

46. Strohbehn, John W.: Line-of-Sight Wave Propagation Through the Turbulent Atmosphere. Proc. IEEE, vol. 56, no. 8, Aug. 1968, pp. 1301-1318.

47. Sutton, O. G.: Micrometeorology. McGraw-Hill Book Co., Inc., 1953.

48. Hinze, J. O.: Turbulence. McGraw-Hill Book Co., Inc., 1959.

49. Schmidt, Dieter W.: Experimental Investigations on the Scattering of Sound by Turbulence. AFOSR 1666, ASTIA Doc. No. AD 266 564, U.S. Air Force, July 1961.

50. Monk, Robin G.: Thermal Relaxation in Humid Air. J. Acoust. Soc. Amer., vol. 46, no. 3, pt. 2, Sept 1969, pp. 580-586.

51. Gething, J. T.: Acoustic Scattering From Atmospheric Inhomogeneities. Publ. No. 17, Project EAR Rep. No. VI, Meteorol. Dep., Univ. of Melbourne, Dec. 1970.

52. Anon.: Noise Standards: Aircraft Type Certification. Federal Aviation Regulations, vol. III, pt. 36, FAA, Dec. 1969.

53. McAllister, L. G.: Acoustic Sounding of the Lower Troposphere. J. Atmos, \& Terrest. Phys., vol. 30, no. 7, July 1968, pp. 1439-1440.

54. McAllister, L. G.; and Pollard, J. R.: Acoustic Sounding of the Lower Atmosphere. Proceedings of Sixth International Symposium on Remote Sensing of Environment, Vol. 1, Inst. Sci. Technol., Univ. of Michigan, Oct. 1969, pp. 436-450.

55. Derr, V. E.; and Little, C. G.: A Comparison of Remote Sensing of the Clear Atmosphere by Optical, Radio, and Acoustic Radar Techniques. Appl. Optics, vol. 9, no. 9 , Sept. 1970 , pp. 1976-1992.

56. Reynolds, M.: The Design and Construction of an Acoustic Sounder. Publ. No. 15, Project EAR Rep. No. III, Meteorol. Dep., Univ. of Melbourne, Apr. 1970.

57. Simmons, W. R.; Wescott, J. W.; and Hall, F. F., Jr.: Acoustic Echo Sounding as Related to Air Pollution in Urban Environments. NOAA TR ERL 216-WPL 17, U.S. Dep. Com., May 1971. 
58. Wescott, J. W.; Simmons, W. R.; and Little, C. G.: Acoustic Echo-Sounding Measurements of Temperature and Wind Fluctuations. ESSA ERLTM-WPL 5, U.S, Dep. Com., Jan. 1970.

59. Hall, F. F., Jr.: Report on the July 1972 Workshop in Atmospheric Acoustics. NOAA TM ERL WPL-8, U.S. Dep. Com., Oct. 1973. 